AECEVIED

SEP 20 ใด

OSTI

Lithology and Stratigraphy of Holes Drilled in LANL-Use Areas of the Nevada Test Site

Volume XIII

1991 - 1998

July 1999 


\section{CREDIT STATEMENT}

Available to the public from:

U. S. Department of Commerce

National Technical Information Service

5285 Port Royal Road

Springfield, Virginia 22161-0002

Telephone (703) $487-4650$

Available electronically at http://www.doe.gov/bridge. Available to U.S. Department of Energy and its contractors in paper from:

U.S. Department of Energy

Office of Scientific and Technical Information

P.O. Box 62

Oak Ridge, TN 37831-0062

(423) $576-8401$

\section{DISCLAIMER STATEMENT}

This report was prepared as an account of work sponsored by an agency of the U.S. Government. Neither the U.S. Government nor any agency thereof, nor any of their employees, nor any of their contractors, subcontractors, or their employees, makes any warranty, express or implied, or assumes any legal liability or responsibility for the accuracy, completeness, or any third party's use or the results of such use of any information, apparatus, product, or process disclosed, or represents that its use would not infringe privately owned rights. Reference herein to any specific commercial product, process, or service by trade name, trademark, manufacturer, or otherwise, does not necessarily constitute or imply its endorsement, recommendation, or favoring by the U.S. Government or any agency thereof or its contractors or subcontractors. The views and opinions of authors expressed herein do not necessarily state or reflect those of the U.S. Government or any agency thereof. 


\section{DISCLAIMER}

Portions of this document may be illegible in electronic image products. Images are produced from the best available original document. 


\title{
Lithology and Stratigraphy of Holes Drilled in LANL-Use Areas of the Nevada Test Site
}

\author{
Volume XIII. \\ 1991 - 1998
}

\author{
Lance B. Prothro \\ Sigmund L. Drellack, Jr. \\ Brian M. Allen \\ Bechtel Nevada Geology/Hydrology Group
}

July 1999

Work performed under contract DE-AC08-96NV11718. 
This page intentionally left blank. 


\section{TABLE OF CONTENTS}

PAGE

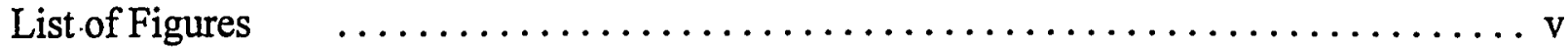

List of Tables $\quad \ldots \ldots \ldots \ldots \ldots \ldots \ldots \ldots \ldots \ldots \ldots \ldots \ldots \ldots \ldots \ldots \ldots \ldots \ldots$ vi

List of Acronyms and Abbreviations $\ldots \ldots \ldots \ldots \ldots \ldots \ldots \ldots \ldots \ldots \ldots \ldots \ldots \ldots \ldots$ vii

Abstract $\ldots \ldots \ldots \ldots \ldots \ldots \ldots \ldots \ldots \ldots \ldots \ldots \ldots \ldots \ldots \ldots \ldots \ldots \ldots$ viii

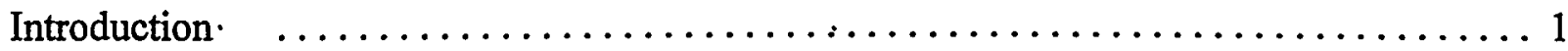

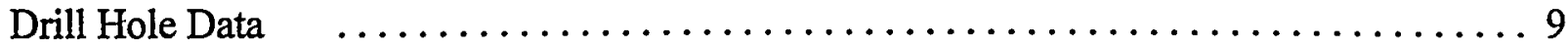

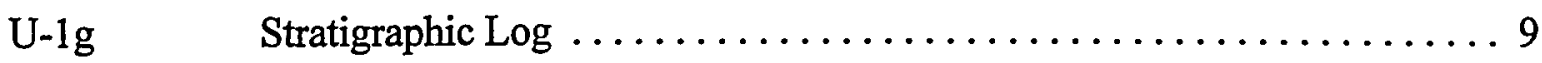

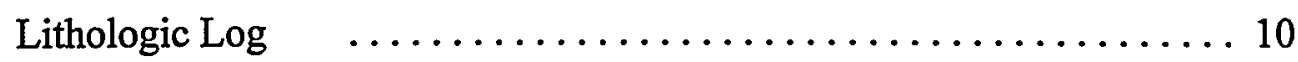

Supplemental Data ........................... 12

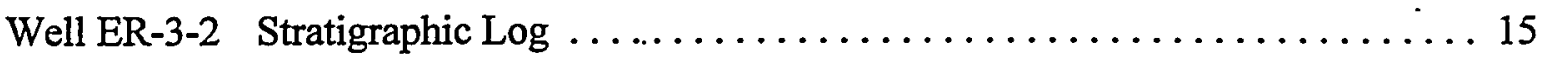

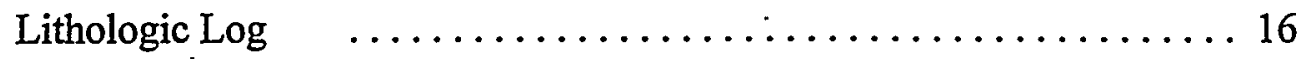

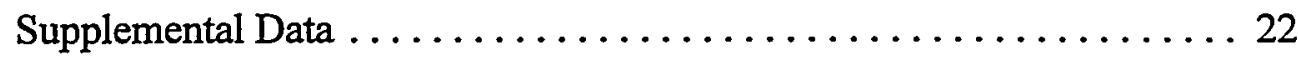

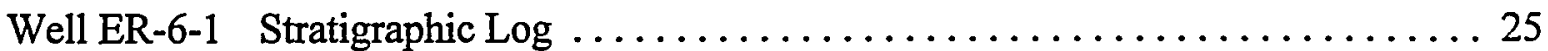

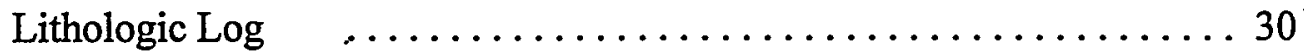

Supplemental Data ......................... 47

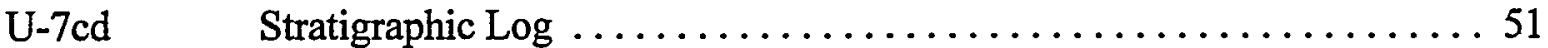

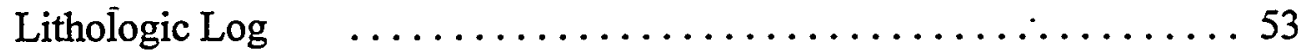

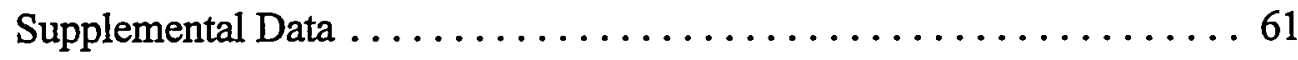

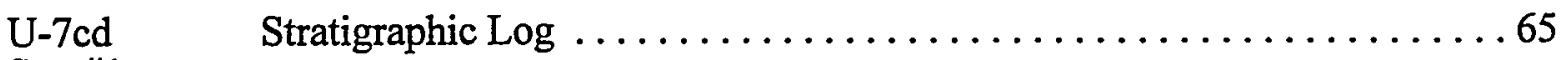

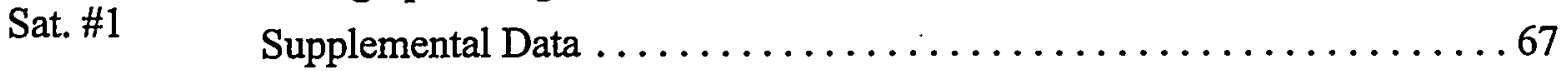

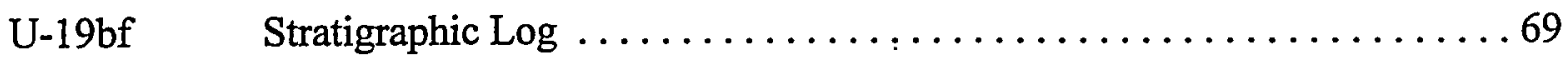

Supplemental Data ........................... 70

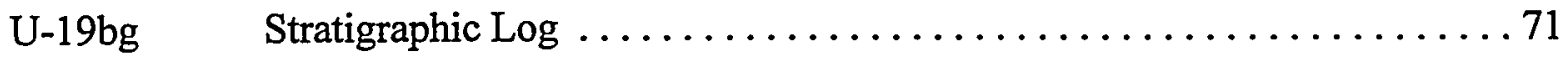

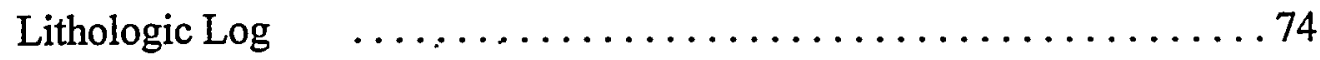

Supplemental Data .............................. 89 


\section{TABLE OF CONTENTS (continued)}

PAGE

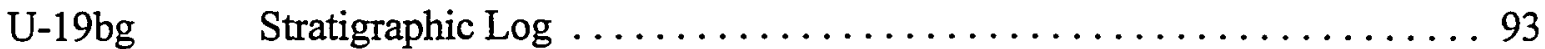

Sat. \#1

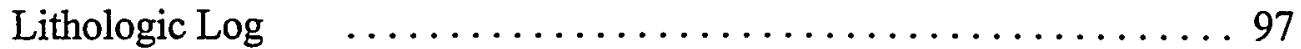

Supplemental Data ........................... 98

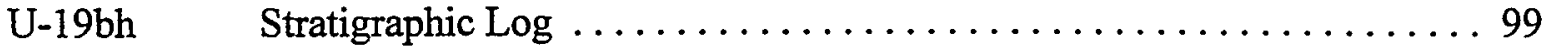

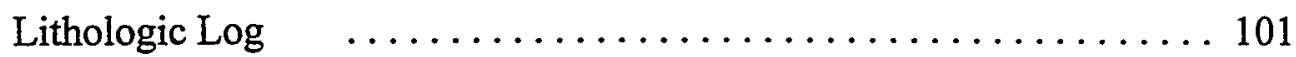

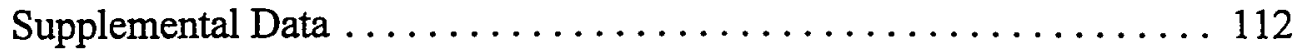

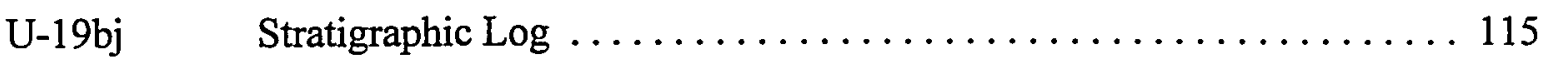

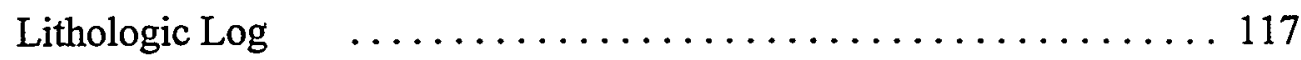

Supplemental Data ......................... 125

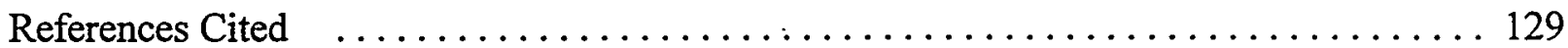




\section{LIST OF FIGURES}

FIGURE

PAGE

1 Map of Holes Drilled in LANL-Use Areas of the Nevada Test Site, Calendar Years 1991 Through 1995 ....................... 4

2 West-East Geologic Cross Section Through U-1g/U1a Complex ......... 13

3 West-East Geologic Cross Section Through ER-3-2 $\ldots \ldots \ldots \ldots \ldots \ldots \ldots$

4 West-East Geologic Cross Section Through Well ER-6-1 . . . . . . . . . . 48

$5 \quad$ North-South Geologic Cross Section Through Well ER-6-1 ............ 49

6 West-East Geologic Cross Section Through U-7cd ............... 62

$7 \quad$ North-South Geologic Cross Section Through U-7cd ............... 63

8 West-East Geologic Cross Section Through U-19bg .............. 90

$9 \quad$ North-South Geologic Cross Section Through U-19bg . . . . . . . . . . . 91

10 West-East Geologic Cross Section Through U-19bh $\ldots \ldots \ldots \ldots \ldots \ldots \ldots$

11 North-South Geologic Cross Section Through U-19bh .............. 114

12 West-East Geologic Cross Section Through U-19ba $\ldots \ldots \ldots \ldots \ldots \ldots \ldots$

13 North-South Geologic Cross Section Through U-19ba ............. 127

Plate Graphic Data Summaries for U-1g, U-7cd, and U-19bh with Lithology, Stratigraphy, and Selected Geophysical Logs ............ Back Pocket 


\section{LIST OF TABLES}

TABLE

PAGE

1 Recent Shallow Boreholes in Area $3 \ldots \ldots \ldots \ldots \ldots \ldots \ldots \ldots$

2 Construction Data for Holes Included in this Report $\ldots \ldots \ldots \ldots \ldots \ldots$

3 Stratigraphic Nomenclature Used in This Report $\ldots \ldots \ldots \ldots \ldots \ldots \ldots$ 


\section{LIST OF ACRONYMS AND ABBREVIATIONS}

$\begin{array}{ll}\text { BN } & \text { Bechtel Nevada } \\ \mathrm{cm} & \text { centimeter } \\ \text { DOE/NV } & \text { U.S. Department of Energy, Nevada Operations Office } \\ \mathrm{ft} & \text { foot (feet) } \\ \text { LANL } & \text { Los Alamos National Laboratory } \\ \text { LLNL } & \text { Lawrence Livermore National Laboratory } \\ \mathrm{m} & \text { meter(s) } \\ \mathrm{mm} & \text { millimeter(s) } \\ \text { NTS } & \text { Nevada Test Site } \\ \text { RSN } & \text { Raytheon Services Nevada } \\ \text { TD } & \text { Total Depth } \\ \text { TDR } & \text { Time Domain Reflectometry } \\ \text { UGTA } & \text { Underground Test Area } \\ \text { USGS } & \text { U.S. Geological Survey }\end{array}$




\title{
Lithology and Stratigraphy of Holes Drilled in LANL-Use Areas of the Nevada Test Site Volume XIII, 1991-1998
}

\begin{abstract}
Geologic data for ten holes drilled in areas used by Los Alamos National Laboratory at the Nevada Test Site are presented in this report. The holes include emplacement holes, instrumentation holes, and Underground Test Area wells drilled during calendar years 1991 through 1995. For each hole a stratigraphic log, a detailed lithologic log, and one or two geologic cross sections are presented, along with a supplemental data sheet containing information about the drilling operations, geology, or references. For three of the holes, graphic data summary sheets with geologic and geophysical data are provided as plates.
\end{abstract}

Keywords: Nevada Test Site, Yucca Flat, Pahute Mesa, geology, alluvium, tuff. 


\title{
Lithology and Stratigraphy of Holes Drilled in \\ LANL-Use Areas of the Nevada Test Site Volume XIII
}

\author{
L. B. Prothro, S. L. Drellack, Jr., and B. M. Allen
}

\section{INTRODUCTION}

This report presents a compilation of data from ten holes drilled during calendar years 1991 through 1995 in areas used by Los Alamos National Laboratory (LANL) at the Nevada Test Site (NTS), Nye County, Nevada. Volume XIII is the latest in a series of reports produced by the Los Alamos Support Group of the Bechtel Nevada (BN) Geology/Hydrology Section and its predecessors. A primary purpose of this series of reports has been to provide readily accessible geologic data originated by BN (and predecessor) geologists for LANL drill holes at the NTS. Volumes I through XII contain information for holes completed in LANL-use areas of Yucca Flat (portions of Areas 1, 3, 4, 6, and 7) between.August 1975 and December 1990. Holes completed prior to August 1975 are described in several U.S. Geological Survey (USGS) open-file reports ("blue books") and are listed in the earlier volumes of this series. Between 1982 and 1990, reports generally were produced on an annual basis. However, this publishing effort has been reduced due to the cessation of nuclear testing: fewer holes were drilled in the 1991-1995 interval, and none have been drilled in former test areas assigned to LANL since 1995. The need for future volumes is assessed on an annual basis and the decision to produce any additional volumes will depend upon the number of holes drilled in LANL-use areas of the NTS.

Volume XIII is the third to include drill holes in LANL-use areas of Pahute Mesa (Area 19). LANL holes on Pahute Mesa completed prior to 1989 will be compiled in a future volume(s). This edition marks the first time that geologic cross sections have been included for each drill hole. One or two cross sections are presented for most of the drill holes included in this report: a west-east section (typically perpendicular to structural trends) and a north-south section (typically parallel to structure).

\section{DRILL HOLE DATA}

Volume XIII provides geologic data for drill holes U-1g, Well ER-3-2, Well ER-6-1, U-7cd, U-7cd Satellite Hole \#1, U-19bf, U-19bg, U-19bg Satellite Hole \#1, U-19bh, and U-19bj. Although expended Emplacement Hole U-19bg was drilled in 1990, the detailed geologic characterization was not finalized until 1991 and so is included in this report. Emplacement Hole 
U-19bf was also drilled in 1990, but was abandoned due to severe sloughing. Due to the limited usefulness of U-19bf and the small amount of data available for the hole, it was not included in Volume XII but is included in Volume XIII for completeness.

Emplacement Holes U-7cd, U-19bh, and U-19bj are inventory holes. The U-1g drill hole is a cable access/emergency egress hole associated with the U-1a Complex (Allen, 1995).

Wells ER-3-2 and.ER-6-1 were drilled for the U.S. Department of Energy, Nevada Operations (DOE/NV) Environmental Restoration Division, and are now part of the Underground Test Area (UGTA) monitoring network. These holes are included because they are located in areas of Yucca Flat assigned to LANL. Several shallow boreholes were drilled in Area 3 within the last few years in support of various DOE/NV environmental restoration projects at the NTS (Table 1). These boreholes are relatively shallow and penetrate only alluvium, so additional details are not included in this report.

The following data are included for each drill hole in this report whenever available:

- Stratigraphic log with abbreviated lithologic descriptions

- Detailed lithologic log,

- Supplemental data (e.g., information on sample analyses, water levels, etc.)

- West-east geologic cross section

- North-south geologic cross section

- Graphic presentation of lithology, stratigraphy and the corresponding responses of selected geophysical logs.

Figure 1 is an index map of the NTS showing locations of drill holes presented in this report. Table 2 lists the state plane coordinates and selected construction data for each drill hole. Stratigraphic nomenclature used in this report is generally from Ferguson and others (1994) and is presented in Table 3. Terminology used in detailed lithologic descriptions (including quantities of rock constituents) is the same as that outlined in Volume $\mathrm{X}$ (drill holes completed in 1988). The lithologic descriptions presented here follow BN Geology/Hydrology Department Procedure NTS-GEO-003. Locations of stratigraphic contacts and lithologic divisions are verified with geophysical log data whenever possible. Other data, such as paleontological analyses, are incorporated where available. 
Table 1

Recent Shallow Boreholes in Area 3

\begin{tabular}{|c|c|c|c|c|}
\hline Borehole Name & $\begin{array}{c}\text { Total Depth } \\
\text { (Measured Depth } \\
\text { in meters }[\mathrm{m}] \text { and } \\
\text { feet }[\mathrm{ft}] \text { ) }\end{array}$ & $\begin{array}{c}\text { Hole } \\
\text { Orientation } \\
\text { (Degrees from } \\
\text { Vertical) } \\
\end{array}$ & $\begin{array}{l}\text { Year } \\
\text { Drilled }\end{array}$ & Project \\
\hline $\begin{array}{l}\text { UE-3bL-D1 } \\
\text { UE-3bL-D2 } \\
\text { UE-3bL-U1 }\end{array}$ & $\begin{array}{l}73.8 \mathrm{~m}(242 \mathrm{ft}) \\
146.3 \mathrm{~m}(480 \mathrm{ft}) \\
76.2 \mathrm{~m}(250 \mathrm{ft})\end{array}$ & $\begin{array}{l}45 \\
45 \\
45\end{array}$ & $\begin{array}{l}1994 \\
1994 \\
1994\end{array}$ & $\begin{array}{l}\text { Performance Assessment of } \\
\text { the Area } 3 \text { Radioactive Waste } \\
\text { Management Site (Schmeltzer } \\
\text { et al., 1996). }\end{array}$ \\
\hline ER-3-3 & $129.5 \mathrm{~m}(425 \mathrm{ft})$ & 6 & $\begin{array}{l}1994 / \\
1995\end{array}$ & $\begin{array}{l}\text { Site characterization for closure } \\
\text { of the U-3fi Injection Well. }\end{array}$ \\
\hline
\end{tabular}




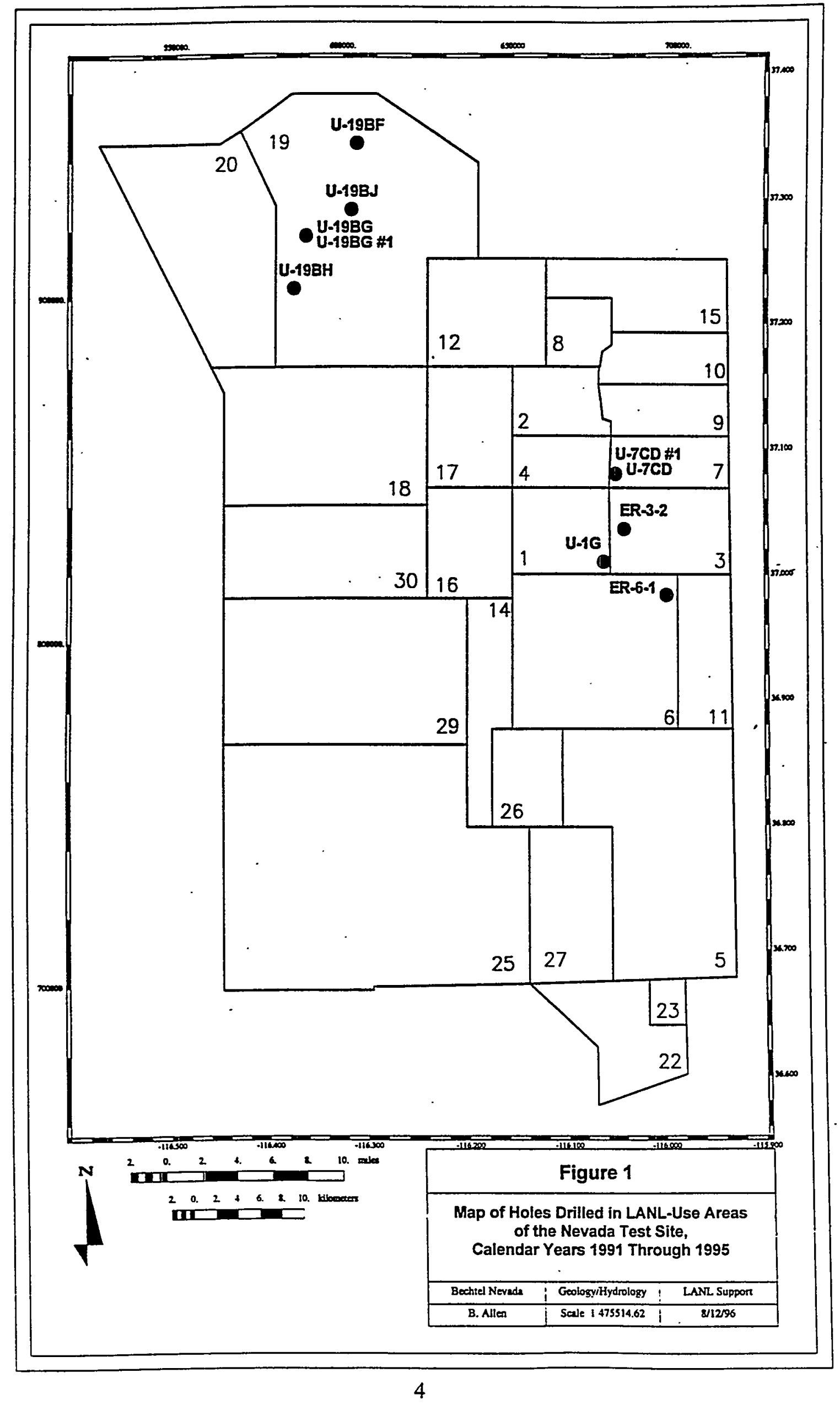


Table 2

Construction Data for Holes Included in this Report

\begin{tabular}{|c|c|c|c|c|c|c|c|}
\hline \multirow{3}{*}{$\begin{array}{c}\text { Hole Name } \\
\text { U-1g }\end{array}$} & \multirow{2}{*}{\multicolumn{2}{|c|}{$\begin{array}{c}\text { State Plane Coordinates } \\
\text { (Nevada Central) } \\
\text { meters } \quad \text { feet }\end{array}$}} & \multicolumn{2}{|c|}{ Surface Elevation } & \multicolumn{2}{|c|}{ Total Depth (TD) } & \multirow{3}{*}{$\begin{array}{c}\begin{array}{c}\text { Date TD } \\
\text { Reached }\end{array} \\
11 / 20 / 1992\end{array}$} \\
\hline & & & meters & feet & meters & feet & \\
\hline & $\begin{array}{l}\text { N 251,034 } \\
\text { E 206,563 }\end{array}$ & $\begin{array}{l}N 823,600 \\
E 677,700\end{array}$ & 1220.2 & 4003.4 & 304.8 & 1000 & \\
\hline ER-3-2 & $\begin{array}{l}\text { N } 253,932.92 \\
E 208,488.12\end{array}$ & $\begin{array}{l}\text { N 833,111.6 } \\
\text { E 684,014.8 }\end{array}$ & 1221.6 & 4007.9 & 914.4 & 3000 & 02/08/1994 \\
\hline ER-6-1 & $\begin{array}{l}\text { N 248, } 108.89 \\
\text { E 212,384.95 }\end{array}$ & $\begin{array}{l}\text { N } 814,003.93 \\
\text { E 696,799.63 }\end{array}$ & 1200.9 & 3940 & 977.2 & 3206 & $10 / 16 / 1994$ \\
\hline U-7cd & $\begin{array}{l}\text { N } 258,775.3 \\
\text { E 207,690.7 }\end{array}$ & $\begin{array}{l}\text { N } 849,000.24 \\
\text { E } 681,399.94\end{array}$ & 1254.2 & 4114.7 & 495.3 & 1625 & $04 / 14 / 1992$ \\
\hline U-7cd Sat \#1 & $\begin{array}{l}\text { N } 258,775.7 \\
\text { E } 207,700.0\end{array}$ & $\begin{array}{l}\text { N } 849,001.66 \\
\text { E } 681,430.57\end{array}$ & 1254.1 & 4114.4 & 518.2 & 1700 & 09/15/1992 \\
\hline$U-19 b f$ & $\begin{array}{l}\text { N 288,079.24 } \\
\text { E 184,223.31 }\end{array}$ & $\begin{array}{l}N 945,140 \\
E 604,406\end{array}$ & 2081.5 & 6829 & 471.2 & 1546 & $06 / 07 / 1990$ \\
\hline$U-19 b g$ & $\begin{array}{l}\text { N 279,898.31 } \\
\text { E 179,617.37 }\end{array}$ & $\begin{array}{l}\text { N } 918,299.72 \\
\text { E } 589,294.68\end{array}$ & 2039.52 & 6691.35 & 657.5 & 2157 & $04 / 20 / 1990$ \\
\hline U-19bg Sat \#1 & $\begin{array}{l}\text { N 279,887.41 } \\
\text { E 179,617.30 }\end{array}$ & $\begin{array}{l}\text { N } 918,263.96 \\
\text { E } 589,294.45\end{array}$ & 2040.3 & 6693.8 & 685.8 & 2250 & $07 / 15 / 1991$ \\
\hline U-19bh & $\begin{array}{l}\text { N } 275,205.1 \\
\text { E } 178,521.7\end{array}$ & $\begin{array}{l}N 902,902 \\
E 585,700\end{array}$ & 2062.9 & 6768 & 654.7 & 2148 & $06 / 14 / 1991$ \\
\hline$U-19 b j$ & $\begin{array}{l}\text { N 282,215.34 } \\
\text { E183,764.65 }\end{array}$ & $\begin{array}{l}\text { N 925,901.53 } \\
\text { E 602,901.20 }\end{array}$ & 2140.9 & 7024 & 656.2 & 2153 & 06/02/1992 \\
\hline
\end{tabular}


Table 3

Stratigraphic Nomenclature Used in this Report

(Modified from Ferguson et al., 1994)

\begin{tabular}{|c|c|}
\hline Stratigraphic Symbol & Stratigraphic Unit ${ }^{\mathrm{a}}$ \\
\hline QTa & Quaternary and Tertiary Alluvium \\
\hline Tt & Thirsty Canyon Group \\
\hline TH & Trail Ridge Tuff \\
\hline Ttp & Pahute Mesa Tuff \\
\hline Tf & Volcanics of Fortymile Canyon \\
\hline Tfbr & rhyolite of Chukar Canyon \\
\hline Tfbw & rhyolite of Beatty Wash \\
\hline Tm & Timber Mountain Group \\
\hline Tma & Ammonia Tanks Tuff \\
\hline $\operatorname{Tma}(b)$ & Ammonia Tanks Tuff (bedded) \\
\hline Tmar & mafic-rich Ammonia Tanks Tuff \\
\hline Tmap & mafic-poor Ammonia Tanks Tuff \\
\hline Tmab & bedded Ammonia Tanks Tuff \\
\hline Tmr & Rainier Mesa Tuff \\
\hline Tmrb & bedded Rainier Mesa Tuff \\
\hline Tmrr & mafic-rich Rainier Mesa Tuff \\
\hline Tmrp & mafic-poor Rainier Mesa Tuff \\
\hline Tmrh & tuff of Holmes Road \\
\hline $\mathrm{Tmrl} / \mathrm{Th}$ & pre-Rainier Mesa, post-Wahmonie bedded tuff, undifferentiated \\
\hline$T p$ & Paintbrush Group \\
\hline$T p b$ & rhyolite of Benham \\
\hline$T p c$ & . Tiva Canyon Tuff \\
\hline Tpcm & Pahute Mesa lobe of Tiva Canyon Tuff \\
\hline Tpd & rhyolite of Delirium Canyon \\
\hline Tpe & rhyolite of Echo Peak \\
\hline $\mathrm{Tpr}$ & rhyolite of Silent Canyon \\
\hline Tpt & Topopah Spring Tuff \\
\hline Tptm & Pahute Mesa lobe of Topopah Spring Tuff \\
\hline
\end{tabular}


Table 3

Stratigraphic Nomenclature Used in this Report (continued)

(Modified from Ferguson et al., 1994)

\begin{tabular}{|c|c|}
\hline Stratigraphic Symbol. & Stratigraphic Unit ${ }^{a}$ \\
\hline Th & Calico Hills Formation \\
\hline Thp & mafic-poor Calico Hills Formation \\
\hline Tw & Wahmonie Formation \\
\hline Twlb & tuff of Wahmonie Flat \\
\hline Tc & Crater Flat Group \\
\hline Tci & rhyolite of Inlet \\
\hline Tcp & Prow Pass Tuff \\
\hline Teps & rhyolite of Sled \\
\hline Tcpk & rhyolite of Kearsarge \\
\hline Tcg & andesite of Grimy Gulch \\
\hline Tcb & Bullfrog Tuff \\
\hline Tct & Tram Tuff \\
\hline Tcl & lower tuff \\
\hline Tb & Belted Range Group \\
\hline Tbd & Dead Horse Flat Formation \\
\hline Tbg & Grouse Canyon Tuff \\
\hline Tbgb & bedded Grouse Canyon Tuff \\
\hline Tn & Tunnel Formation \\
\hline $\operatorname{Tn} 4$ & Tunnel 4 Member \\
\hline Tn4K & beds $4 \mathrm{~K}$ \\
\hline Tn4J & beds $4 \mathrm{~J}$ \\
\hline Tn4FGH & beds $4 \mathrm{FGH}$, undifferentiated \\
\hline Tn4E & beds $4 \mathrm{E}$ (equivalent) \\
\hline $\operatorname{Tn} \triangle A B C D$ & beds $\triangle A B C D$, undifferentiated \\
\hline Tn3 & Tunnel 3 Member \\
\hline $\operatorname{Tn} 3 \mathrm{D}$ & beds 3D \\
\hline Tn3BC & beds $3 B C$ (equivalent) \\
\hline $\operatorname{Tn} 3 \mathrm{~A}$ & beds $3 \mathrm{~A}$ \\
\hline
\end{tabular}


Table 3

Stratigraphic Nomenclature Used in this Report (continued)

(Modified from Ferguson et al., 1994)

\begin{tabular}{||l|l||}
\hline Stratigraphic Symbol & \multicolumn{1}{|c|}{ Stratigraphic Unit $^{\text {a }}$} \\
\hline \hline Tu & Volcanics of Big Dome \\
\hline Tub & Tub Spring Tuff \\
\hline Ton2 & $\begin{array}{l}\text { Volcanics of Oak Spring Butte (symbol also used for older tuffs, } \\
\text { undifferentiated) }\end{array}$ \\
\hline Toy & tunnel bed 2 \\
\hline Ton1 & Yucca Flat Tuff \\
\hline TI tunnel bed 1 \\
\hline Tlc & Paleocolluvium \\
\hline Pz & tuffaceous Paleocolluvium \\
\hline Dg & Paleozoic Sedimentary Rocks \\
\hline Ds & Guilmette Formation \\
\hline DV & Simonson Dolomite \\
\hline SI & Sevy Dolomite \\
\hline Oes & Laketown Dolomite \\
\hline Oe & Ely Springs Dolomite \\
\hline Oa & Eureka Quartzite \\
\hline On & Antelope Valley Limestone \\
\hline
\end{tabular}

Notes

a The units shown are in stratigraphic order, however, the list does not represent the complete stratigraphic sequence of the NTS. 
Stratigraphic Log of Drill Hole U-1g

(Logged by Brian M. Allen and Sigmund L. Drellack, Jr., Raytheon Services Nevada, December 1992)

\begin{tabular}{|c|c|c|c|c|c|c|}
\hline \multicolumn{2}{|c|}{ Depth } & \multirow[t]{2}{*}{ Lithology } & \multirow[t]{2}{*}{ Stratigraphic Unit } & \multirow[t]{2}{*}{ Symbol } & \multicolumn{2}{|c|}{ Thickness } \\
\hline Meters & Feet & & & & Meters & Feet \\
\hline $\begin{array}{c}0-304.8 \\
\text { TD }\end{array}$ & $\begin{array}{c}0-1,000 \\
\text { TD }\end{array}$ & Tuffaceous Alluvium & Alluvium & QTa & 304.8 & 1000 \\
\hline
\end{tabular}




\section{Lithologic Log of Drill Hole U-1g}

(Logged by B. M. Allen and S. L. Drellack, Jr., Raytheon Services Nevada)

December 1992

Unless otherwise noted, the following descriptions refer to washed cuttings samples at 3.05-meter (m) (10-foot [ft]) intervals. Colors are determined by comparing wet sample color to the Geological Society of America Rock-Color Chart. Stratigraphic contacts and lithologic divisions are tied to geophysical logs whenever possible.

Depth

Lithologic Description

Stratigraphic Unit

Meters/(feet)

$0-36.6 \mathrm{~m}$

$(0-120 \mathrm{ft})$

$36.6-115.8 \mathrm{~m}$

$(120-380 \mathrm{ft})$
Alluvium: No samples collected.

Alluvium

Alluvium: Dominantly tuffaceous; grayish-orange (10YR7/4) to darkyellowish-orange (10YR6/6);

moderately to poorly indurated, moderately to poorly sorted, subrounded to subangular; average approximately $5-10$ percent Paleozoic fragments with about 1 - 5 percent (of total sample) carbonates; moderately calcareous with caliche rinds on some grains; some $\mathrm{MnO}_{2}$ dendrites on clasts; slight increase in Paleozoic rock content in $54.9-\mathrm{m}(180-\mathrm{ft}), 67.1-\mathrm{m}$ (220-ft), and 91.4-m (300-ft) samples.
Alluvium 


\begin{tabular}{ccc}
\hline $\begin{array}{c}\text { Depth } \\
\text { Meters/(feet) }\end{array}$ & Lithologic Description & Stratigraphic Unit \\
\hline
\end{tabular}

$115.8-304.8 \mathrm{~m}$ $(380-1,000 \mathrm{ft})$

TD
Alluvium: Dominantly tuffaceous; moderate-yellowish-brown (10YR5/4) to light-brown (5YR5/6); moderately to poorly indurated, moderately to poorly sorted, subrounded to subangular; approximately $25-40$ percent Paleozoic rock fragments (increasing gradually with depth). Paleozoic rock fraction is mostly grayish-black (N2) to brownish-black (5YR2/1) argillite and quartzite; carbonate percentage (of total sample) is approximately 5 10 percent. Slight increase in Paleozoic rock percentage noticed in $125-\mathrm{m}$ (410-ft), 259-m (850-ft), and 280.4-m (920-ft) samples; carbonate portion of total sample increases gradually with depth to approximately 15 percent from $292.6 \mathrm{~m}$ ( $960 \mathrm{ft}$ ) to TD. Common to abundant caliche rinds and $\mathrm{MnO}_{2}$ dendrites on larger grains; slight increase in moderateyellow-brown (10YR5/4) tuff at $27.1 .3 \mathrm{~m}(890 \mathrm{ft})$ and ash-flow detritus(?) at $207.3 \mathrm{~m}(680 \mathrm{ft})$.

Alluvium 


\section{Drill Hole U-1g Supplemental Data}

1. For additional information regarding this site, see Allen (1994). Supplemental data listed below are also extracted from Allen (1994), unless noted otherwise.

2. No sidewall sampling has been performed to date.

3. Downhole photography available: Lawrence Livermore National Laboratory (LLNL) black-and-white format video. Two downhole runs were performed, one along the northwest portion of the borehole, and the other along the southeast portion of the borehole.

4. Static water level at the U-1g location is estimated to be at a depth of $\sim 478 \mathrm{~m}(1,568 \mathrm{ft})$ which equates to an elevation of $742 \mathrm{~m}(2,435 \mathrm{ft})$. This water level depth is within the alluvium section, but is well below the TD of the U-1g drill hole.

5. The U-1g borehole was designed and constructed primarily as a cable access hole. The Lyner Complex vent drift intersects the U-1g borehole, with the invert level located at a depth of $296 \mathrm{~m}$ (972 ft) below ground level (Allen, 1995). U-1g also serves as an emergency-egress and ventilation-line passageway for the Lyner Complex.

6. See graphic data summary sheet in back pocket. 


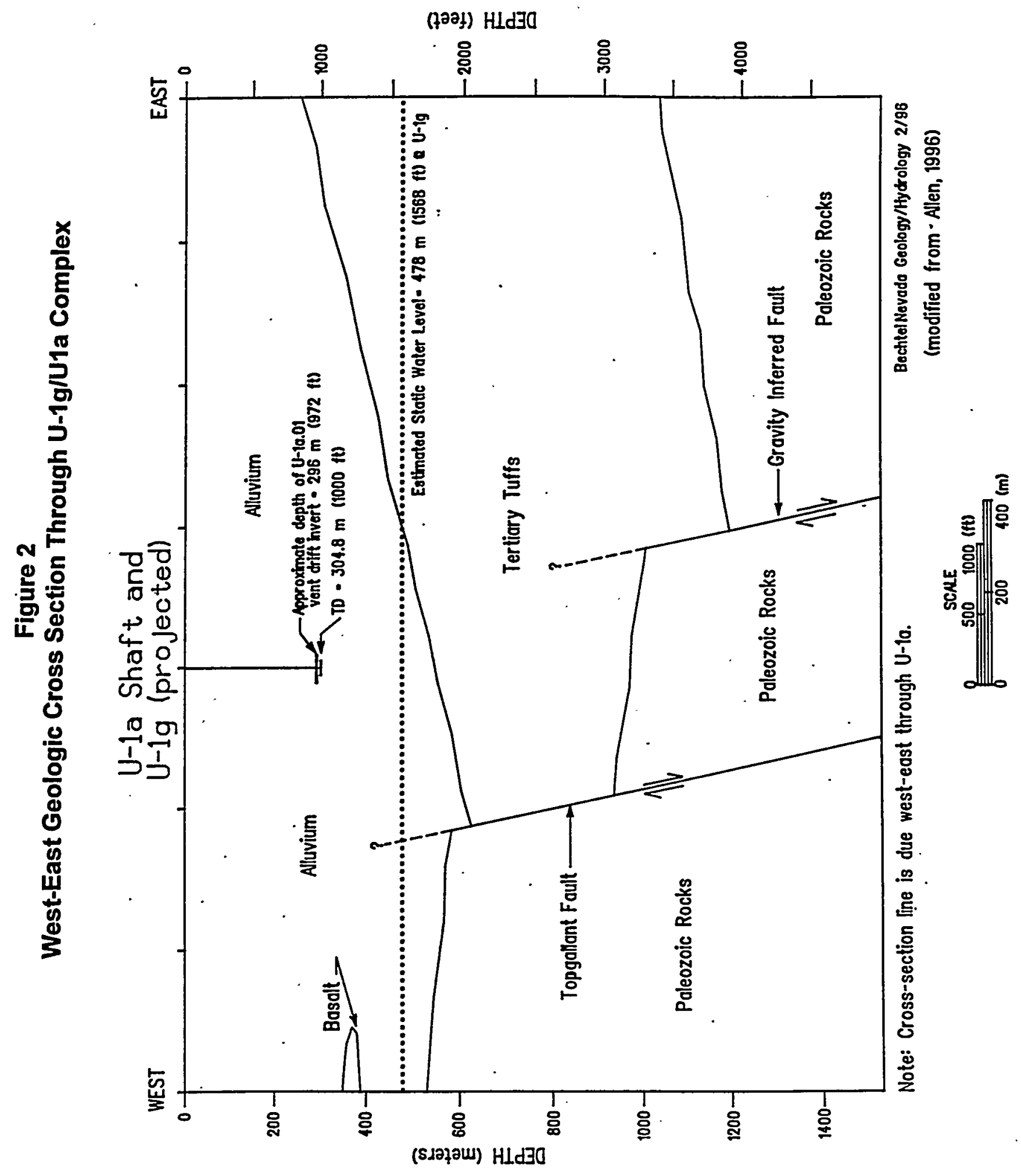


This page intentionally left blank. 
Stratigraphic Log of Well ER-3-2

(Logged by Lance B. Prothro, Raytheon Services Nevada, 24 April 1995)

\begin{tabular}{|c|c|c|c|c|c|c|}
\hline \multicolumn{2}{|c|}{ Depth } & Lithology & Stratigraphic Unit & Symbol & \multicolumn{2}{|c|}{ Thickness } \\
\hline $0-801.9$ & $0-2,631$ & $\begin{array}{l}\text { Sand, Gravelly Sand, } \\
\text { and Sandy Gravel: Fine } \\
\text { sand to very coarse } \\
\text { pebble gravel, poorly to } \\
\text { moderately sorted. }\end{array}$ & Alluvium & QTa & 801.9 & 2631 \\
\hline $801.9-846.1$ & $2,631-2,776$ & $\begin{array}{l}\text { Ash-Flow Tuff: } \\
\text { Nonwelded to partially } \\
\text { welded, vitric. }\end{array}$ & $\begin{array}{l}\text { Ammonia Tanks Tuff, } \\
\text { Timber Mountain Group }\end{array}$ & Tma & 44.2 & 145 \\
\hline $846.1-851.9$ & $2,776-2,795$ & Bedded Tuff: Vitric. & $\begin{array}{l}\text { bedded } \\
\text { Ammonia Tanks Tuff, } \\
\text { Timber Mountain Group }\end{array}$ & Tmab & 5.8 & 19 \\
\hline $\begin{array}{c}851.9-914.4 \\
\text { TD }\end{array}$ & $\begin{array}{c}2,795-3,000 \\
\text { TD }\end{array}$ & $\begin{array}{l}\text { Ash-Flow Tuff: } \\
\text { Nonwelded to } \\
\text { moderately welded, vitric } \\
\text { to devitrified. }\end{array}$ & $\begin{array}{l}\text { Rainier Mesa Tuff, } \\
\text { Timber Mountain Group }\end{array}$ & Tmr & $>62.5$ & $>205$ \\
\hline
\end{tabular}




\section{Lithologic Log of Well ER-3-2 \\ Compiled by Lance B. Prothro, Raytheon Services Nevada \\ April 1995}

Unless otherwise noted, the following descriptions refer to washed cuttings samples at $3.05-\mathrm{m}$ (10-ft) intervals. The lithologic descriptions follow Raytheon Services Nevada Department Procedure NTS-GEO-003, "Description of Rock Samples" (RSN, 1993), and were compiled using the IT Corp. field sample descriptions. In addition, petrographic analysis, quantitative $X$-ray diffraction analysis, and sample descriptions provided by R. G. Warren (LANL) were also used. Stratigraphic contacts and lithologic divisions are tied to geophysical logs whenever possible.

\begin{tabular}{ccc}
\hline $\begin{array}{c}\text { Depth } \\
\text { Meters/(Feet) }\end{array}$ & Lithologic Description & Stratigraphic Unit \\
\hline
\end{tabular}

$0-115.8 \mathrm{~m}$

$(0-380 \mathrm{ft})$
Interbedded Sandy Gravel and

Gravelly Sand: Samples are unconsolidated mixtures of the coarser clasts that make up the alluvium.

Samples are moderate-yellowish-brown in overall color; consist of varying amounts of medium to coarse sand and very fine to medium pebble gravel; poorly to moderately sorted; subangular to subrounded. Gravel and coarse sand fractions consist predominately of tuffaceous clasts and much less siltstone clasts. The smaller sandstone fraction consists of tuffaceous clasts and quartz and feldspar crystals. Samples indicate sandy gravel intervals at 39.6 -

$51.8 \mathrm{~m}(130-170 \mathrm{ft}), 73.2-85.3 \mathrm{~m}$

$(240-280 \mathrm{ft}), 91.4-100.6 \mathrm{~m}$ $(300-330 \mathrm{ft})$, and $109.7-115.8 \mathrm{~m}(360-$ $380 \mathrm{ft})$.
Alluvium 


\begin{tabular}{ccc}
\hline $\begin{array}{c}\text { Depth } \\
\text { Meters/(Feet) }\end{array}$ & Lithologic Description & Stratigraphic Unit \\
\hline
\end{tabular}

$115.8-320.0 \mathrm{~m}$ $(380-1,050 \mathrm{ft})$

$320.0-466.3 \mathrm{~m}$ $(1,050-1,530 \mathrm{ft})$
Sandy Gravel: Samples are unconsolidated mixtures of the coarser clasts that make up the alluvium. Samples are moderate-yellowish-brown in overall color; consist predominately of fine to medium pebble gravel; moderately sorted; subangular to subrounded. Gravel consists predominately of tuffaceous clasts and lesser siltstone, carbonate, and argillite clasts. Fine pebble gravel dominates samples to $146.3 \mathrm{~m}$ ( $480 \mathrm{ft}$ ) becoming mostly medium pebble gravel below.

Gravelly Sand: Samples are unconsolidated mixtures of the coarser clasts that make up the alluvium. Samples are moderate-yellowish-brown in overall color; consist predominately of medium to very coarse sand and lesser very fine to fine pebble gravel; poorly to moderately sorted; subangular to subrounded. Sand and gravel consist predominately of tuffaceous clasts and lesser siltstone, carbonate, and argillite clasts. Quartz and feldspar crystals make up a portion of the smaller sand fraction. Samples and geophysical logs indicate minor interbedded sandy gravel to $368.8 \mathrm{~m}(1,210 \mathrm{ft})$ and below $432.8 \mathrm{~m}$ $(1,420 \mathrm{ft})$.
Alluvium

Alluvium 


\begin{tabular}{ccc}
\hline $\begin{array}{c}\text { Depth } \\
\text { Meters/(Feet) }\end{array}$ & Lithologic Description & Stratigraphic Unit \\
\hline
\end{tabular}

$466.3-533.4 \mathrm{~m}$

$(1,530-1,750 \mathrm{ft})$

$533.4-588.0 \mathrm{~m}$

$(1,750-1,929 \mathrm{ft})$
Sandy Gravel: Samples are unconsolidated mixtures of the coarser clasts that make up the alluvium with very minor amounts of intact alluvium. Samples are moderate-yellowish-brown (based on intact fragments of alluvium); consist predominately of very fine to medium pebble gravel increasing in size to coarse pebble gravel near base; moderately sorted, becoming poorly sorted near base; subangular to subrounded. Clasts are mostly tuffaceous with lesser siltstone, carbonate, and sedimentary breccia. Intact fragments of alluvium are moderately cemented with calcite.

Sandy Gravel: Samples are unconsolidated mixtures of the coarser clasts that make up the alluvium with minor amounts of intact alluvium. Samples are moderate-yellowish-brown (based on intact fragments of alluvium); consist predominately of fine to very coarse pebble gravel (very coarse pebble gravel is conspicuous); poorly sorted; subangular to subrounded. Gravel consists predominately of tuffaceous clasts with lesser sedimentary clasts representing a variety of lithologies. Intact fragments of alluvium are moderately cemented with calcite.
Alluvium

Alluvium 
Depth

Meters/(Feet)
Lithologic Description

Stratigraphic Unit

$588.0-801.9 \mathrm{~m}$

$(1,929-2,631 \mathrm{ft})$

$801.9-837.3 \mathrm{~m}$

$(2,631-2,747 \mathrm{ft})$
Tuffaceous Sand: Description is based on percussion gun sidewall core samples. Samples are predominately moderateyellowish-brown; tuffaceous; mostly vitric, commonly zeolitic, as well as argillaceous and silicified in part; fine to medium sand; moderately sorted; subangular to subrounded; consisting predominately of tuffaceous rock and pumice clasts, quartz and feldspar crystals, and lesser mafic minerals; minor cross-bedded laminae.

Partially Welded Ash-Flow Tuff: Description is based on percussion gun sidewall core samples. Moderateyellowish-brown to approximately $807.7 \mathrm{~m}(2,650 \mathrm{ft})$ becoming darkyellowish-brown below; vitric; common very-light-gray and lesser dark-yellowishorange pumice to approximately $807.7 \mathrm{~m}$ $(2,650 \mathrm{ft})$ becoming minor in abundance and moderate-brown (5YR4/4) in color below; common felsic phenocrysts of feldspar and lesser quartz; rare mafic minerals of clinopyroxene and hornblende, and lesser biotite; rare lithic fragments; trace of sphene; conspicuous black glass shards. Tuff/Alluvium contact determined from sidewall core samples and gamma ray $\log \# 13$.
Alluvium

mafic-poor

Ammonia Tanks Tuff 
Lithologic Log of Well ER-3-2 (cont.)

\begin{tabular}{|c|c|c|}
\hline $\begin{array}{c}\text { Depth } \\
\text { Meters/(Feet) }\end{array}$ & Lithologic Description & Stratigraphic Unit \\
\hline $\begin{array}{l}837.3-846.1 \mathrm{~m} \\
(2,747-2,776 \mathrm{ft})\end{array}$ & $\begin{array}{l}\text { Nonwelded Ash-Flow Tuff: Description } \\
\text { is based on percussion gun sidewall core } \\
\text { samples. Very-pale-orange; vitric; rare to } \\
\text { common white pumice; common to } \\
\text { abundant felsic phenocrysts of feldspar } \\
\text { and lesser quartz; rare tiny mafic minerals } \\
\text { of clinopyroxene, hornblende, and lesser } \\
\text { biotite; rare lithic fragments. }\end{array}$ & $\begin{array}{c}\text { mafic-poor } \\
\text { Ammonia Tanks Tuff }\end{array}$ \\
\hline $\begin{array}{l}846.1-851.9 \mathrm{~m} \\
(2,776-2,795 \mathrm{ft})\end{array}$ & $\begin{array}{l}\text { Bedded Tuff: Description is based on } \\
\text { percussion gun sidewall core samples. } \\
\text { Pale- to moderate-yellowish-brown; } \\
\text { vitric; rare to common white pumice; } \\
\text { common to abundant felsic phenocrysts of } \\
\text { feldspar and lesser quartz; rare tiny mafic } \\
\text { minerals of clinopyroxene, hornblende, } \\
\text { and lesser biotite; rare lithic fragments. }\end{array}$ & $\begin{array}{c}\text { bedded } \\
\text { Ammonia Tanks Tuff }\end{array}$ \\
\hline & $\cdot$ & \\
\hline $\begin{array}{l}851.9-877.2 \mathrm{~m} \\
(2,795-2,878 \mathrm{ft})\end{array}$ & $\begin{array}{l}\text { Nonwelded to Partially Welded Ash- } \\
\text { Flow Tuff: Description is based on } \\
\text { percussion gun sidewall core samples. } \\
\text { Grayish-orange-pink; vitric, zeolitic in } \\
\text { part; rare white pumice; common felsic } \\
\text { phenocrysts of feldspar and lesser but } \\
\text { conspicuous quartz; common mafic } \\
\text { minerals of biotite and lesser } \\
\text { clinopyroxene and hornblende; rare lithic } \\
\text { fragments. }\end{array}$ & $\begin{array}{c}\text { mafic-rich } \\
\text { Rainier Mesa Tuff }\end{array}$ \\
\hline
\end{tabular}


Lithologic Log of Well ER-3-2 (cont.)

\begin{tabular}{|c|c|c|}
\hline $\begin{array}{c}\text { Depth } \\
\text { Meters/(Feet) }\end{array}$ & Lithologic Description & Stratigraphic Unit \\
\hline $\begin{array}{l}877.2-897.9 \mathrm{~m} \\
\left(2,878^{\circ}-2,946 \mathrm{ft}\right)\end{array}$ & $\begin{array}{l}\text { Moderately Welded Ash-Flow Tuff: } \\
\text { Description is based on percussion gun } \\
\text { sidewall core samples. Pale-yellowish- } \\
\text { brown; devitrified; common white } \\
\text { pumice; rare to common felsic } \\
\text { phenocrysts of feldspar and lesser quartz; } \\
\text { rare to common mafic minerals of large } \\
\text { biotite and much less clinopyroxene; rare } \\
\text { lithic fragments. }\end{array}$ & $\begin{array}{c}\text { mafic-rich } \\
\text { Rainier Mesa Tuff }\end{array}$ \\
\hline $\begin{array}{c}897.9-914.4 \mathrm{~m} \\
(2,946-3,000 \mathrm{ft}) \\
\text { TD }\end{array}$ & $\begin{array}{l}\text { Moderately Welded Ash-Flow Tuff: } \\
\text { Description is based on percussion gun } \\
\text { sidewall core samples. Grayish-pink; } \\
\text { devitrified; minor pale-red pumice; rare to } \\
\text { common felsic phenocrysts of feldspar } \\
\text { and quartz; rare biotite; trace lithic } \\
\text { fragments. }\end{array}$ & $\begin{array}{l}\text { mafic-poor } \\
\text { Rainier Mesa Tuff }\end{array}$ \\
\hline
\end{tabular}




\section{Well ER-3-2 Supplemental Data}

1. For additional information regarding Well ER-3-2, see DOE (1995). Supplemental data listed below are also extracted from DOE (1995).

2. Well ER-3-2 is part of the UGTA monitoring well network.

3. Time Domain Reflectometry (TDR) instrumentation used to detect the presence of water in the drilling effluent was tested by Desert Research Institute during drilling of Well ER-3-2. Analysis of TDR and drilling data indicated a water level depth of $490 \mathrm{~m}$ $(1,608 \mathrm{ft})$.

4. Drill cuttings were collected continuously at $3.05 \mathrm{~m}$ (10 ft) intervals from $36.6-603.5 \mathrm{~m}$ $(120-1,980 \mathrm{ft})$. However, due to lost circulation conditions below $603.5 \mathrm{~m}(1,980 \mathrm{ft})$, drill cuttings were obtained from only the $612.6 \mathrm{~m}(2,010 \mathrm{ft})$ and $623.6 \mathrm{~m}(2,046 \mathrm{ft})$ depths. Because of the lack of drill cuttings samples below $603.5 \mathrm{~m}(1,980 \mathrm{ft}), 66$ percussion gun sidewall samples were collected from 611.7 to $911.4 \mathrm{~m}(2,007-2,990 \mathrm{ft})$.

5. Drilling of Well ER-3-2 took longer than expected because the target formation was deeper than predicted, and because of complications due to unstable hole conditions. Shortly after the water table was penetrated, the saturated alluvium began to slough. Consequently, no open hole pre-completion development was attempted in Well ER-3-2. 
Figure 3

West-East Geologic Cross Section Through ER-3-2

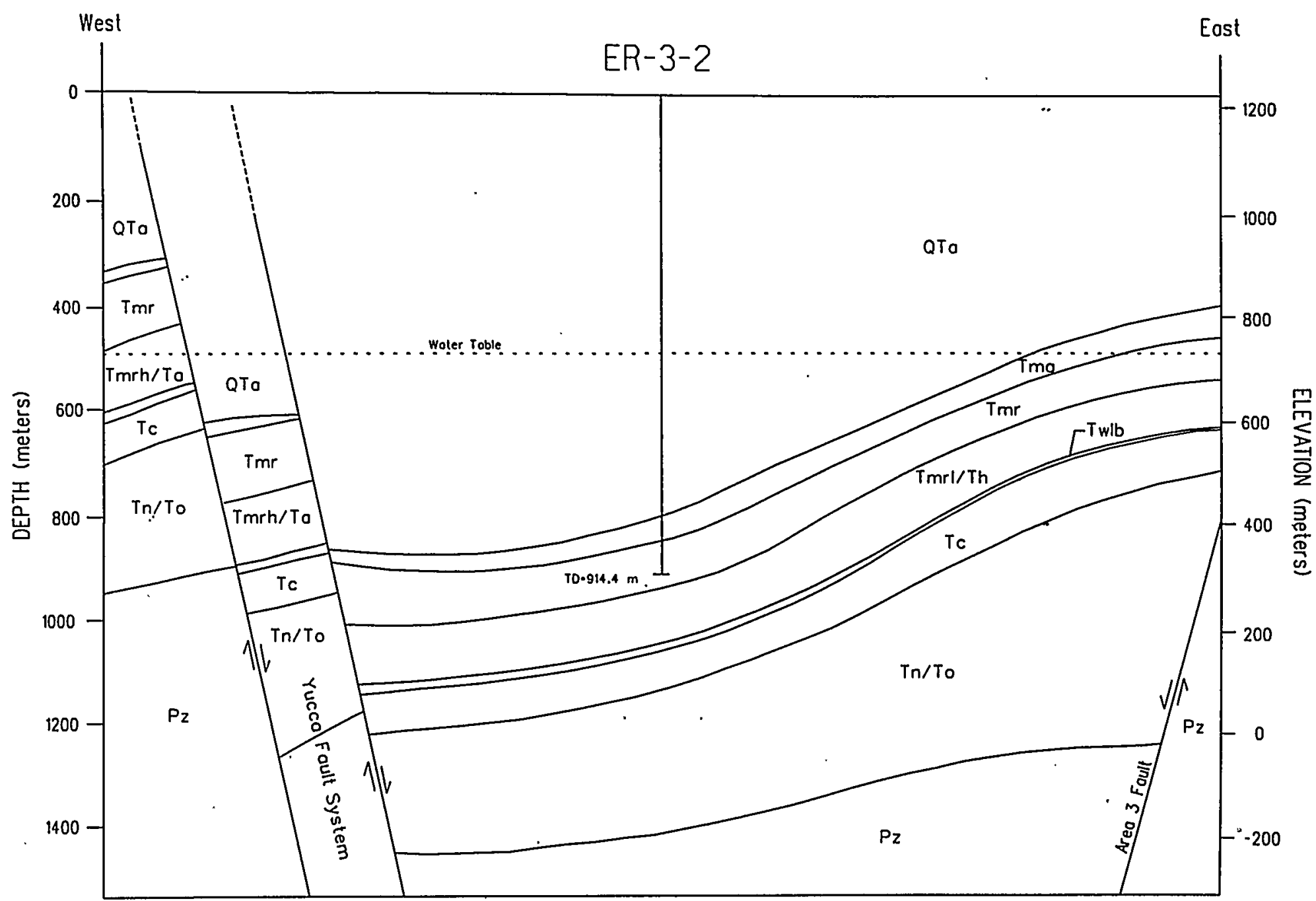

KEY TO STRATIGRAPHIO SYMBOLS

QTo - Quoternory/Tertiory Aluvium

Tmo - Ammonia Tanks Tuff (includes Tmab)

Tmr - Roinier Meso Tull

Tmrl/Th - Pre-Rainier Mesa, post-Wohmonie

tuffs, undifferentioted
Twlb - Tuff of Wohmonie Flat

Tc - Croter Flot lufl's, undifferentiated

Tn/To - Tunnelformation and Older Volconics. undiffer entioted

$P_{Z}$ - Poleozoic Rocks, undifferentioled
SCALE (meters)

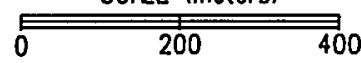

(modified from DOE, 1995) 
This page intentionally left blank. 


\begin{tabular}{|c|c|c|c|c|c|c|}
\hline \multirow[b]{2}{*}{ Meters } & Depth & \multirow[t]{2}{*}{ Lithology } & \multirow[t]{2}{*}{ Stratigraphic Unit } & \multirow[t]{2}{*}{ Symbol } & \multicolumn{2}{|c|}{ Thickness } \\
\hline & Feet & & & & Meters & Feet \\
\hline $0-101.5$ & $0-333$ & Tuffaceous Alluvium & Alluvium & QTa & 101.5 & 333 \\
\hline $101.5-134.1$ & $333-440$ & $\begin{array}{l}\text { Ash-Flow Tuff: } \\
\text { Nonwelded to partially } \\
\text { welded, vapor-phase to } \\
111.3 \mathrm{~m} \text { ( } 365 \mathrm{ft} \text { ), vitric } \\
\text { below. }\end{array}$ & $\begin{array}{c}\text { mafic-poor } \\
\text { Rainier Mesa Tuff, } \\
\text { Timber Mountain Group }\end{array}$ & Tmrp & 32.6 & 107 \\
\hline $134.1-137.2$ & $440-450$ & Reworked Tuff: Vitric. & $\begin{array}{l}\text { tuff of Holmes Road, } \\
\text { Timber Mountain Group }\end{array}$ & Tmrh & 3.1 & 10 \\
\hline $137.2-152.7$ & $450-501$ & $\begin{array}{l}\text { Ash-Flow Tuff: } \\
\text { Nonwelded to partially } \\
\text { welded, vitric to silicified. }\end{array}$ & $\begin{array}{l}\text { Tiva Canyon Tuff, } \\
\text { Paintbrush Group }\end{array}$ & $\mathrm{Tpc}$ & 15.5 & 51 \\
\hline $152.7-166.1$ & $501-5 \dot{4} 5$ & Bedded Tuff: Zeolitized. & $\begin{array}{l}\text { Paintbrush Group, } \\
\text { undifferentiated }\end{array}$ & $\mathrm{Tp}$ & 13.4 & 44 \\
\hline $166.1-237.1$ & $545-778$ & $\begin{array}{l}\text { Ash-Flow Tuff: } \\
\text { Nonwelded to densely } \\
\text { welded, vitric to devitrified. }\end{array}$ & $\begin{array}{l}\text { Topopah Spring Tuff, } \\
\text { Paintbrush Group }\end{array}$ & Tpt & 71.0 & 233 \\
\hline $237.1-251.8$ & $778-826$ & $\begin{array}{l}\text { Bedded Tuff: Vitric, } \\
\text { zeolitized below } 240.2 \mathrm{~m} \\
(788 \mathrm{ft}) .\end{array}$ & $\begin{array}{c}\text { mafic-poor } \\
\text { Calico Hills Formation }\end{array}$ & Thp & 14.7 & 48 \\
\hline
\end{tabular}


Stratigraphic Log of Well ER-6-1 (cont.)

\begin{tabular}{llllll}
\hline \hline Depth & Lithology & Stratigraphic Unit & Symbol & Thickness \\
Meters & Feet & & Feters & Meters \\
\hline \hline
\end{tabular}

\begin{tabular}{|c|c|c|c|c|c|}
\hline $251.8-285.3$ & $826-936$ & $\begin{array}{l}\text { Bedded Tuff: Zeolitized, } \\
\text { mafic rich. }\end{array}$ & tuff of Wahmonie Flat & Twlb ${ }^{1}$ & 33.5 \\
\hline $285.3-307.2$ & 936.- 1008 & Air-Fall Tuff: Zeolitized. & $\begin{array}{l}\text { Prow Pass Tuff, } \\
\text { Crater Flat Group }\end{array}$ & Tcp & 21.9 \\
\hline $307.2-324.9$ & $1,008-1,066$ & $\begin{array}{l}\text { Ash-Flow Tuff: Partially } \\
\text { welded, zeolitized. }\end{array}$ & $\begin{array}{l}\text { Bullfrog Tuff, } \\
\text { Crater Flat Group }\end{array}$ & $\mathrm{Tcb}$ & 17.7 \\
\hline $324.9-333.8$ & $1,066-1,095$ & Bedded Tuff: Zeolitized. & $\begin{array}{c}\text { Tram Tuff, } \\
\text { Crater Flat Group }\end{array}$ & Tet & 8.9 \\
\hline $333.8-353.0$ & $1,095-1,158$ & Air-Fall Tuff: Zeolitized. & $\begin{array}{c}\text { lower tuff, } \\
\text { Crater Flat Group }\end{array}$ & $\mathrm{Tcl}$ & 19.2 \\
\hline $353.0-381.0$ & $1,158-1,250$ & Air-Fall Tuff: Zeolitized. & $\begin{array}{c}\text { Tunnel Formation, } \\
\text { Tunnel } 4 \text { Member, } \\
\text { beds } 4 \mathrm{~K}\end{array}$ & $\operatorname{Tn} 4 \mathrm{~K}$ & 28.0 \\
\hline $381.0-390.1$ & $1,250-1,280$ & Air-Fall Tuff: Zeolitized. & $\begin{array}{l}\text { Tunnel Formation, } \\
\text { Tunnel } 4 \text { Member, } \\
\text { beds } 4 \mathrm{~J}\end{array}$ & $\operatorname{Tn} 4 \mathrm{~J}$ & 9.1 \\
\hline
\end{tabular}


Stratigraphic Log of Well ER-6-1 (cont.)

\begin{tabular}{|c|c|c|c|c|c|c|}
\hline \multicolumn{2}{|c|}{ Depth } & \multirow[t]{2}{*}{ Lithology } & \multirow[t]{2}{*}{ Stratigraphic Unit } & \multirow[t]{2}{*}{ Symbol } & \multicolumn{2}{|c|}{ Thickness } \\
\hline Meters & Feet & & & & Meters & Feet \\
\hline $390.1-392.6$ & $1,280-1,288$ & Air-Fall Tuff: Zeolitized. & $\begin{array}{c}\text { Tunnel Formation, } \\
\text { Tunnel } 4 \text { Member, } \\
\text { beds } 4 \mathrm{FGH}, \\
\text { undifferentiated }\end{array}$ & Tn4FGH & 2.5 & 8 \\
\hline $392.6-401.7$ & $1,288-1,318$ & $\begin{array}{l}\text { Ash-Flow Tuff: } \\
\text { Nonwelded, zeolitized. }\end{array}$ & $\begin{array}{l}\text { Tunnel Formation, } \\
\text { Tunnel } 4 \text { Member, } \\
\text { beds 4E (equivalent) }\end{array}$ & $\operatorname{Tn} 4 \mathrm{E}$ & 9.1 & 30 \\
\hline $401.7-405.4$ & $1,318-1,330$ & Bedded Tuffs: Zeolitized. & $\begin{array}{c}\text { Tunnel Formation, } \\
\text { Tunnel } 4 \text { Member, } \\
\text { beds } 4 A B C D, \\
\text { undifferentiated }\end{array}$ & $\operatorname{Tn} 4 A B C D$ & 3.7 & ${ }^{\prime} 12$ \\
\hline . $405.4-409.7$ & $1,330-1,344$ & $\begin{array}{l}\text { Bedded Tuff: Zeolitized to } \\
\text { weakly argillized. }\end{array}$ & $\begin{array}{c}\text { Tunnel Formation, } \\
\text { Tunnel } 3 \text { Member, } \\
\text { beds 3D }\end{array}$ & $\operatorname{Tn} 3 \mathrm{D}$ & 4.3 & 14 \\
\hline $409.7-414.5$ & $1,344-1,360$ & $\begin{array}{l}\text { Ash-Flow Tuff: } \\
\text { Nonwelded, zeolitized. }\end{array}$ & $\begin{array}{l}\text { Tunnel Formation, } \\
\text { Tunnel } 3 \text { Member, } \\
\text { beds 3BC (equivalent) }\end{array}$ & $\operatorname{Tn} 3 B C^{2}$ & 4.8 & 16 \\
\hline $414.5-429.2$ & $1,360-1,408$ & Bedded Tuff: Zeolitized. & $\begin{array}{c}\text { Tunnel Formation, } \\
\text { Tunnel } 3 \text { Member, } \\
\text { beds } 3 \mathrm{~A}\end{array}$ & $\operatorname{Tn} 3 \mathrm{~A}$ & 14.7 & 48 \\
\hline
\end{tabular}


Stratigraphic Log of Well ER-6-1 (cont.)

\begin{tabular}{|c|c|c|c|c|c|c|}
\hline \multicolumn{2}{|c|}{ Depth } & \multirow[t]{2}{*}{ Lithology } & \multirow[t]{2}{*}{ Stratigraphic Unit } & \multirow[t]{2}{*}{ Symbol } & \multicolumn{2}{|c|}{ Thickness } \\
\hline Meters & Feet & & & & Meters & Feet \\
\hline $429.2-432.2$ & $1,408-1,418$ & $\begin{array}{l}\text { Air-Fall Tuff: Peralkaline, } \\
\text { zeolitized. }\end{array}$ & $\begin{array}{l}\text { Tub Spring Tuff, } \\
\text { Volcanics of Big Dome }\end{array}$ & Tub & 3.0 & 10 \\
\hline $432.2-455.7$ & $1,418-1,495$ & Bedded Tuff: Zeolitized. & $\begin{array}{c}\text { tunnel bed 2, } \\
\text { Volcanics of Oak Spring Butte }\end{array}$ & Ton2 & 23.5 & 77 \\
\hline $455.7-492.9$ & $1,495-1,617$ & $\begin{array}{l}\text { Ash-Flow Tuff: } \\
\text { Nonwelded, zeolitized. }\end{array}$ & $\begin{array}{l}\text { Yucca Flat Tuff, } \\
\text { Volcanics of Oak Spring Butte }\end{array}$ & Toy & 37.2 & 122 \\
\hline $492.9-522.4$ & $1,617-1,714$ & Bedded Tuff: Zeolitized. & $\begin{array}{l}\text { tunnel bed 1, } \\
\text { Volcanics of Oak Spring Butte }\end{array}$ & Ton 1 & 29.5 & 97 \\
\hline $522.4-538.0$ & $1,714-1,765$ & $\begin{array}{l}\text { Bedded Tuff: Zeolitized to } \\
\text { argillized. }\end{array}$ & $\begin{array}{l}\text { older tuffs, } \\
\text { undifferentiated }\end{array}$ & To & 15.6 & 51 \\
\hline $538.0-539.5$ & $1,765-1,770$ & $\begin{array}{l}\text { Paleocolluvium: Paleozoic } \\
\text { carbonate clasts in a } \\
\text { tuffaceous argillized } \\
\text { matrix. }\end{array}$ & $\begin{array}{c}\text { tuffaceous } \\
\text { Paleocolluvium }\end{array}$ & Tlc & 1.5 & 5 \\
\hline $539.5-680.9$ & $1,770-2,234$ & $\begin{array}{l}\text { Dolostone: Light-olive- } \\
\text { gray to light-brownish-gray, } \\
\text { fine to medium crystalline. }\end{array}$ & Sevy Dolomite & Dv & 141.4 & 464 \\
\hline
\end{tabular}


Stratigraphic Log of Well ER-6-1 (cont.)

\begin{tabular}{|c|c|c|c|c|c|c|}
\hline \multicolumn{2}{|c|}{ Depth } & \multirow[t]{2}{*}{ Lithology } & \multirow[t]{2}{*}{ Stratigraphic Unit } & \multirow[t]{2}{*}{ Symbol } & \multicolumn{2}{|c|}{ Thickness } \\
\hline Meters & Feet & & & & Meters & Feet \\
\hline $680.9-890.0$ & $2,234-2,920$ & $\begin{array}{l}\text { Dolostone: Alternating } \\
\text { intervals of light-brownish- } \\
\text { gray, finely crystalline, } \\
\text { vuggy dolostone; and } \\
\text { medium-dark-gray, } \\
\text { medium-crystalline, cherty } \\
\text { dolostone. }\end{array}$ & Laketown Dolomite & Sl & 209.1 & 686 \\
\hline $890.0-940.6$ & $2,920-3,086$ & $\begin{array}{l}\text { Dolostone: Medium-gray } \\
\text { to dark-gray and pale- } \\
\text { yellowish-brown, medium- } \\
\text { crystalline, cherty. }\end{array}$ & Ely Springs Dolomite & Oes & 50.6 & 166 \\
\hline $\begin{array}{c}940.6-977.2 \\
\text { TD }\end{array}$ & $\begin{array}{c}3,086-3,206 \\
\text { TD }\end{array}$ & $\begin{array}{l}\text { Sandstone and Quartzite: } \\
\text { Sandstone is light- } \\
\text { brownish-gray to brownisl- } \\
\text { gray, fine- to medium- } \\
\text { grained, well sorted, and } \\
\text { dolomitic. Quartzite is } \\
\text { light-brownish-gray and } \\
\text { very-pale-orange, medium- } \\
\text { grained, and well sorted. }\end{array}$ & Eureka Quartzite & $\mathrm{Oe}$ & $>36.6$ & $>120$ \\
\hline
\end{tabular}

1 Preliminary work for this site reinforces interpretations based on petrographic work by R. G. Warren (LANL) for the U-3mL site that complex Tw/Ta or Tc relationships exist in this area. Additional work is needed to understand this relationship.

2 Recent studies indicate that the informal unit "Tunnel Formation, Subunit 3BC-Equivalent" may be the Tuff of Tolicha Peak (verbal communication, R. G. Warren). 


\author{
Lithologic Log of Well ER-6-1 \\ Compiled by Lance B. Prothro, Bechtel Nevada \\ June 1996
}

Lithologic descriptions for the interval $0-648.9 \mathrm{~m}(2,129 \mathrm{ft})$ are from Drellack and others (1992) and were compiled by Sigmund L. Drellack Jr. and Robert L. McCall of Raytheon Services Nevada. These descriptions are from drill cuttings samples at 3.0-m (1- $\mathrm{ft})$ intervals. Descriptions below $648.9 \mathrm{~m}(2,129 \mathrm{ft})$ are from IT Corporation (1995) and were compiled by Lance B. Prothro of Raytheon Services Nevada. These descriptions are from 8.4-centimeter (3.3-inch) conventional cores. Only minor modifications for consistency and continuity were made to the original logs. The lithologic descriptions follow Raytheon Services Nevada Department Procedure NTS-GEO-003, "Description of Rock Samples." Stratigraphic contacts and lithologic divisions are tied to geophysical logs whenever possible. Stratigraphic nomenclature is generally from Ferguson and others (1994). Paleontological analysis by J. C. Cole and A. G. Harris of the United States Geological Survey (USGS) (Cole and Harris, 1996) confirmed most of the original stratigraphic assignments below $648.9 \mathrm{~m}(2,129 \mathrm{ft})$. The analysis indicated that the original assignment of Laketown Dolomite for the interval $648.9-680.9 \mathrm{~m}(2,129-2,234 \mathrm{ft})$ is incorrect. The interval is actually Sevy Dolomite and has been reassigned accordingly. Based on this reassignment and previous paleontological work by A. G. Harris (USGS) on drill cuttings from the overlying interval of dolostone (Cole and Harris, 1996), the interval from 539.5 $648.9 \mathrm{~m}(1,770-2,129 \mathrm{ft})$ has also been reassigned to the Sevy Dolomite. Notes 1 and 2 are listed at end of log, page 46.

\title{
Depth Lithologic Description \\ Stratigraphic Unit Meters/(Feet)
}

$0-101.5 \mathrm{~m}$

$(0-333 \mathrm{ft})$

$101.5-111.3 \mathrm{~m}$ $(333-365 \mathrm{ft})$
Tuffaceous Alluvium: Light-brown to moderate-brown (5YR3/4) and moderateyellowish-brown; nonindurated to poorly indurated, rare fairly indurated calichecemented aggregates; poorly sorted, silt to pebble sizes; tuffaceous with less than 2 percent Paleozoic rock fragments; less than 1percent total carbonate fragments; subrounded to subangular; calcareous. Sandy in parts, particularly above $61 \mathrm{~m}$ (200 ft). Granule and larger sized pieces are largely Rainier Mesa Tuff (Tmr) lithology with lesser Topopah Spring Tuff (Tpt) and other pre-Rainier units. Tpt constituent increasing in $67-88 \mathrm{~m}$ (220 $290 \mathrm{ft}$ ) interval.

\section{Partially Welded Ash-Flow Tuff:} Light-gray with purplish tint; devitrified; strong vapor-phase mineralization; fair induration; minor light-brownish-gray to moderate-yellowish-brown pumice; minor dipyramidal quartz and feldspar phenocrysts; scarce mafic minerals; scarce lithic fragments.
Alluvium

mafic-poor

Rainier Mesa Tuff 


\begin{tabular}{|c|c|c|}
\hline $\begin{array}{c}\text { Depth } \\
\text { Meters/(Feet) }\end{array}$ & Lithologic Description & Stratigraphic Unit \\
\hline $\begin{array}{c}111.3-117.3 \mathrm{~m} \\
(365-385 \mathrm{ft})\end{array}$ & $\begin{array}{l}\text { Partially Welded Ash-Flow Tuff: } \\
\text { Grayish-red to moderate-brown; well } \\
\text { indurated; becoming moderate-reddish- } \\
\text { brown and less indurated below } 113 \mathrm{~m} \\
\text { ( } 370 \mathrm{ft} \text { ); vitric; common pale-red pumice; } \\
\text { abundant clear glass shards. }\end{array}$ & $\begin{array}{c}\text { mafic-poor } \\
\text { Rainier Mesa Tuff }\end{array}$ \\
\hline $\begin{array}{c}117.3-128.0 \mathrm{~m} \\
(385-420 \mathrm{ft})\end{array}$ & $\begin{array}{l}\text { Nonwelded Ash-Flow Tuff: Pale- } \\
\text { reddish-brown to moderate-reddish- } \\
\text { brown; friable; vitric; common pumice, } \\
\text { sizes to greater than } 1 \text { centimeter (cm) in } \\
\text { diameter; common quartz and feldspar } \\
\text { phenocrysts; scarce to rare mafic } \\
\text { minerals; rare lithic fragments; very } \\
\text { abundant clear glass shards. }\end{array}$ & $\begin{array}{l}\text { mafic-poor } \\
\text { Rainier Mesa Tuff }\end{array}$ \\
\hline $\begin{array}{c}128.0-134.1 \mathrm{~m} \\
(420-440 \mathrm{ft})\end{array}$ & $\begin{array}{l}\text { Nonwelded Ash-Flow Tuff: Pale- } \\
\text { reddish-brown to moderate-orange-pink; } \\
\text { fair induration to friable; vitric; common } \\
\text { grayish-orange-pink to pinkish-gray } \\
\text { pumice; minor to common quartz and } \\
\text { feldspar phenocrysts; scarce mafic } \\
\text { minerals; rare lithic fragments. }\end{array}$ & $\begin{array}{l}\text { mafic-poor } \\
\text { Rainier Mesa Tuff }\end{array}$ \\
\hline $\begin{array}{c}134.1-137.2 \mathrm{~m} \\
(440-450 \mathrm{ft})\end{array}$ & $\begin{array}{l}\text { Reworked Tuff: Light-brown to } \\
\text { moderate-brown; well indurated; vitric to } \\
\text { devitrified; calcareous to weakly } \\
\text { silicified; rare pumice; common to } \\
\text { abundant feldspar and quartz phenocrysts; } \\
\text { rare small mafic minerals of hornblende, } \\
\text { biotite, and magnetite; rare lithic } \\
\text { fragments. }\end{array}$ & tuff of Holmes Road \\
\hline
\end{tabular}




\begin{tabular}{ccc}
\hline $\begin{array}{c}\text { Depth } \\
\text { Meters/(Feet) }\end{array}$ & Lithologic Description & Stratigraphic Unit \\
\hline
\end{tabular}

$137.2-143.3 \mathrm{~m}$ $(450-470 \mathrm{ft})$

$143.3-149.3 \mathrm{~m}$ $(470-490 \mathrm{ft})$

$149.3-152.7 \mathrm{~m}$ $(490-501 \mathrm{ft})$
Nonwelded Ash-Flow Tuff: Moderateyellowish-brown; moderate induration; vitric; dark-yellowish-orange to darkyellowish-brown pumice to $>2 \mathrm{~cm}$ in diameter; shard rich; scarce feldspar phenocrysts; very scarce mafic minerals, including biotite and clinopyroxene; very scarce lithic fragments.

\section{Nonwelded to Partially Welded Ash-} Flow Tuff: Dark-yellowish-brown; well indurated; vitric (glass shards) to devitrified (matrix) to silicified, some sucrosic secondary mineralization, possibly weakly zeolitized; minor moderate-brown pumice; phenocryst poor; porous texture, voids 0.1 millimeter ( $\mathrm{mm})$ in size.

Nonwelded Ash-Flow Tuff: Moderate to dark-yellowish-brown; poorly indurated, friable; vitric; shard rich; very (Shard-rich base) scarce pumice; phenocryst poor; trace biotite; trace lithic fragments.
Tiva Canyon Tuff

Tiva Canyon Tuff

Tiva Canyon Tuff 


\begin{tabular}{|c|c|c|}
\hline $\begin{array}{c}\text { Depth } \\
\text { Meters/(Feet) }\end{array}$ & Lithologic Description & Stratigraphic Unit \\
\hline $\begin{array}{c}152.7-166.1 \mathrm{~m} \\
(501-545 \mathrm{ft})\end{array}$ & $\begin{array}{l}\text { Bedded Tuff: Grayish-orange and } \\
\text { moderate-yellowish-brown to yellowish- } \\
\text { gray to pale-greenish-yellow; moderately } \\
\text { to well indurated; zeolitized; some } \\
\text { sucrosic secondary mineralization; } \\
\text { common pale-greenish-yellow pumice to } \\
1 \mathrm{~cm} \text { in diameter; scarce feldspar } \\
\text { phenocrysts; scarce to rare mafic } \\
\text { minerals including clinopyroxene, } \\
\text { including distinct small } \mathrm{MnO}_{2} \text { stains, } \\
\text { an increase in mafic minerals around } \\
160 \text { - } 163 \mathrm{~m}(525 \text { - } 535 \mathrm{ft}) ; \text { rare to } \\
\text { minor lithic fragments. }\end{array}$ & $\begin{array}{l}\text { Paintbrush Group, } \\
\text { undifferentiated }\end{array}$ \\
\hline $\begin{array}{c}166.1-169.2 \mathrm{~m} \\
(545-555 \mathrm{ft})\end{array}$ & $\begin{array}{l}\text { Nonwelded Ash-Flow Tuff: Moderate- } \\
\text { yellowish-brown; moderately indurated to } \\
\text { friable; zeolitized; possibly sucrosic } \\
\text { secondary mineralization; common light- } \\
\text { olive-gray to dark-yellowish-brown } \\
\text { pumice; rare felsic phenocrysts; scarce } \\
\text { mafic minerals; very scarce lithic } \\
\text { fragments. }\end{array}$ & Topopah Spring Tuff \\
\hline $\begin{array}{c}169.2-170.7 \mathrm{~m} \\
(555-560 \mathrm{ft})\end{array}$ & $\begin{array}{l}\text { Moderately to Densely Welded Ash- } \\
\text { Flow Tuff: Moderate-brown; very hard } \\
\text { and dense; devitrified; minor feldspar } \\
\text { phenocrysts. }\end{array}$ & Topopah Spring Tuff \\
\hline
\end{tabular}

$170.7-175.3 \mathrm{~m}$ $(560-575 \mathrm{ft})$

Densely Welded Ash-Flow Tuff: Black; vitrophyre; perlitic.glass texture minor to common black flattened pumice; minor feldspar phenocrysts. Some (vitrophyre) mottled with moderate-brown, densely welded, and devitrified.
Topopah Spring Tuff - (Vitrophyre) 


\begin{tabular}{ccc}
\hline $\begin{array}{c}\text { Depth } \\
\text { Meters/(Feet) }\end{array}$ & Lithologic Description & Stratigraphic Unit \\
\hline
\end{tabular}

$175.3-184.1 \mathrm{~m}$ (575 - $604 \mathrm{ft})$

$184.1-213.4 \mathrm{~m}$ $(604-700 \mathrm{ft})$

$213.4-225.6 \mathrm{~m}$ $(700-740 \mathrm{ft})$

$225.6-231.6 \mathrm{~m}$ $(740-760 \mathrm{ft})$
Moderately to Densely Welded AshFlow Tuff: Mottled moderate-brown and grayish-red; devitrified; minor pumice; minor feldspar phenocrysts; very scarce mafic (minerals, including clinopyroxene and biotite; scarce lithic fragments. Conspicuous lithophysae cavities to $5 \mathrm{~cm}$ in diameter noted in downhole camera video. Euhedral quartz clusters with individual crystals $<1 \mathrm{~mm}$ to $3 \mathrm{~mm}$ in length present in cuttings samples.

Moderately to Densely Welded AshFlow Tuff: Pale-brown; very hard and dense; devitrified; rare pumice with some vapor-phase mineralization; rare feldspar phenocrysts; scarce mafic minerals (bronze biotite); very scarce lithic fragments.

Densely Welded Ash-Flow Tuff: Moderate-brown; very hard and dense; vitric to partially devitrified; some anastomosing devitrification ("leopard spots"), dark-grayish-red and moderatebrown; phenocryst poor; very scarce mafic minerals; very scarce lithic fragments.

Moderately Welded Ash-Flow Tuff: Olive-black glass shards in a moderatebrown matrix; well indurated; vitric; "basal vitrophyre;" very abundant translucent olive-black glass shards; phenocryst poor.
Topopah Spring Tuff

(Lithophysal zone)

Topopah Spring Tuff

Topopah Spring Tuff

Topopah Spring Tuff ("Basal Vitrophyre") 
Depth

Meters/(Feet)

$231.6-237.1 \mathrm{~m}$

$(760-778 \mathrm{ft})$

$237.1-240.2 \mathrm{~m}$

$(778-788 \mathrm{ft})$

$240.2-251.8 \mathrm{~m}$ $(788-826 \mathrm{ft})$

$251.8-254.5 \mathrm{~m}$ $(826-835 \mathrm{ft})$
Lithologic Description

Stratigraphic Unit

Nonwelded to Partially Welded Ash-

Flow Tuff: Moderate-yellowish-brown; moderate- to dark-yellowish-brown matrix and very abundant olive-black glass shards; well indurated; vitric; phenocryst poor.

Air-Fall Tuff: Pale-yellow-brown; vitric; moderate induration; abundant clear to translucent very-light-gray glass shards; minor to common light-gray pumice, $3 \mathrm{~mm}$ to several centimeters in diameter, and light-brown pumice, 1 to . $6 \mathrm{~mm}$ in diameter; minor felsic phienocrysts; scarce mafic minerals; rare lithic fragments.

Bedded Tuff: Grayish-orange to moderate-yellowish-brown; moderately to well indurated; zeolitized; generally common to abundant very-pale-orange pumice, common feldspar and quartz phenocrysts; rare mafic minerals; rare to minor lithic fragments.

Bedded Tuff: Moderate-yellowishbrown; moderate induration; zeolitized; minor pumice; mafic rich, mostly biotite, some hornblende; minor lithic fragments.
Topopah Spring Tuff

\author{
mafic-poor \\ Calico Hills Formation
}

\author{
mafic-poor \\ Calico Hills Formation
}

tuff of Wahmonie Flat 


\begin{tabular}{ccc}
\hline $\begin{array}{c}\text { Depth } \\
\text { Meters/(Feet) }\end{array}$ & Lithologic Description & Stratigraphic Unit \\
\hline
\end{tabular}

$254.5-257.6 \mathrm{~m}$ $(835-845 \mathrm{ft})$

$257.6-260.6 \mathrm{~m}$

$(845-855 \mathrm{ft})$

$260.6-269.4 \mathrm{~m}$ $(855-884 \mathrm{ft})$

$269.4-279.8 \mathrm{~m}$ (884 - $918 \mathrm{ft})$
Bedded Tuff: Pale-greenish-yellow to grayish-yellow; good induration; zeolitized; mafic rich, mostly biotite and magnetite, some hornblende, trace clinopyroxene; common to indistinct pale-greenish-yellow pumice; abundant feldspar phenocrysts; rare lithic fragments; possibly interbedded with dark-yellowish-brown reworked tuff and tuffaceous sandstone described below.

Tuffaceous Sandstone: Dark-yellowishbrown; moderate induration; zeolitized; fine grained; bedded; mafic rich, mostly biotite; common small lithic fragments.

NOTE: The 259-m (850-ft) cuttings sample is contaminated with cavings from up-hole.

Air-Fall Tuff: Pale-greenish-yellow; good induration; zeolitized; mafic-rich, mostly biotite; minor feldspar phenocrysts; rare lithic fragments. Possibly some tuffaceous sandstone as above.

Air-Fall Tuff: Grayish-yellow; good induration; zeolitized; abundant to indistinct pale-greenish-yellow pumice; scarce quartz and feldspar phenocrysts; scarce mafic minerals, rare $\mathrm{MnO}_{2}$ stains; minor lithic fragments $0.5 \mathrm{~m}$ to $2 \mathrm{~mm}$ in diameter. Trace botryoidal chalcedony vein material in the $277.4 \mathrm{~m}(910 \mathrm{ft})$ sample. tuff of Wahmonie Flat

tuff of Wahmonie Flat
Crater Flat Group, undifferentiated or Calico Hills Formation ${ }^{1}$




\begin{tabular}{ccc}
\hline $\begin{array}{c}\text { Depth } \\
\text { Meters/(Feet) }\end{array}$ & Lithologic Description & Stratigraphic Unit \\
\hline
\end{tabular}

$279.8-285.3 \mathrm{~m}$

$(918-936 \mathrm{ft})$

$285.3-300.5 \mathrm{~m}$ $(936-986 \mathrm{ft})$

$300.5-307.2 \mathrm{~m}$ $(986-1,008 \mathrm{ft})$

$307.2-324.9 \mathrm{~m}$ $(1,008-1,066 \mathrm{ft})$
Reworked Tuff grading to Tuffaceous Sandstone: Dark-yellowish-brown; good induration; zeolitized; minor small, verypale-orange pumice; mafic-rich; common feldspar phenocrysts; minor small lithic fragments. This lithology is represented in the $286.5-\mathrm{m}$ and $289.6-\mathrm{m}$ (940-ft and 950-ft) cuttings samples.

Bedded Tuff: Grayish-orange; well indurated; zeolitized; common palegreenish-yellow pumice to $1 \mathrm{~cm}$ in diameter; minor to common feldspar and quartz phenocrysts; common small lithic fragments 0.25 to $1 \mathrm{~mm}$ in diameter; rare mafic minerals of biotite, magnetite, trace hornblende.

Bedded Tuff: Grayish-yellow; well indurated; zeolitized; small to indistinct pale-greenish-yellow pumice; abundant feldspar and quartz phenocrysts; minor mafic minerals, biotite dominant, magnetite, trace hornblende; minor lithic fragments, typically 0.5 to $1 \mathrm{~mm}$ in diameter. Grades to tuffaceous sandstone in parts.

Partially Welded Ash-Flow Tuff: Grayish-orange; well indurated; zeolitized; minor to common pumice; rare felsic phenocrysts; rare mafic minerals, mostly biotite, some hornblende; rare lithic fragments; trace silicified in upper $3 \mathrm{~m}(10 \mathrm{ft})$. tuff of Wahmonie Flat ${ }^{1}$

Prow Pass Tuff

Prow Pass Tuff

Bullfrog Tuff 


\begin{tabular}{ccc}
\hline $\begin{array}{c}\text { Depth } \\
\text { Meters/(Feet) }\end{array}$ & Lithologic Description & Stratigraphic Unit \\
\hline
\end{tabular}

$324.9-333.8 \mathrm{~m}$ $(1,066-1,095 \mathrm{ft})$

$333.8-353.0 \mathrm{~m}$ $(1,095-1,158 \mathrm{ft})$

$353.0-364.5 \mathrm{~m}$ $(1,158-1,196 \mathrm{ft})$

$364.5-377.3 \mathrm{~m}$ $(1,196-1,238 \mathrm{ft})$
Air-Fall Tuff: Grayish-yellow; fairly indurated; zeolitized; common grayishyellow to pale-greenish-yellow pumice; minor feldspar and quartz phenocrysts; rare mafic minerals of biotite, hornblende, and magnetite; scarce moderate-brown (5YR4/4) lithic fragments up to $1 \mathrm{~cm}$ in diameter.

\begin{abstract}
Air-Fall Tuff: Moderate-yellowishbrown; well indurated; zeolitized; abundant very pale-orange to palegreenish-yellow pumice 0.5 to $2 \mathrm{~mm}$ in diameter; minor felsic phenocrysts; minor mafic minerals; rare lithic fragments.
\end{abstract}

Bedded Tuff: Moderate-yellowishbrown, some mottled with moderatereddish-brown; moderately indurated; zeolitized; common very-pale-orange pumice; minor felsic phenocrysts; minor to common mafic minerals including biotite, trace pyroxene; rare lithic fragments.
Air-Fall Tuff: Moderate-reddish-brown; well indurated; zeolitized; abundant grayish-orange-pink pumice, 0.5 to $3 \mathrm{~mm}$ Tunnel Formation, Tunnel 4 Member, beds $4 \mathrm{~K}$ in diameter, some with mafic minerals; minor felsic phenocrysts; common mafic minerals, mostly biotite with lesser hornblende; rare lithic fragments.

lower tuff

Tunnel Formation, Tunnel 4 Member, beds $4 \mathrm{~K}$ 


\begin{tabular}{|c|c|c|}
\hline $\begin{array}{c}\text { Depth } \\
\text { Meters/(Feet) }\end{array}$ & Lithologic Description & Stratigraphic Unit \\
\hline $\begin{array}{l}377.3-381.0 \mathrm{~m} \\
(1,238-1,250 \mathrm{ft}) .\end{array}$ & $\begin{array}{l}\text { Air-Fall Tuff: Yellowish-gray to very- } \\
\text { light-gray; fair induration; zeolitized; } \\
\text { mafic-rich, mostly biotite and hornblende; } \\
\text { common felsic phenocrysts; indistinct } \\
\text { very-light-gray pumice; scarce lithic } \\
\text { fragments. }\end{array}$ & $\begin{array}{c}\text { Tunnel Formation, } \\
\text { Tunnel } 4 \text { Member, } \\
\text { beds } 4 \mathrm{~K}\end{array}$ \\
\hline $\begin{array}{c}381.0-390.1 \mathrm{~m} \\
(1,250-1,280 \mathrm{ft})\end{array}$ & $\begin{array}{l}\text { Air-Fall Tuff: Moderate-reddish-brown; } \\
\text { moderately to well indurated; zeolitized } \\
\text { to argillized; common very-pale-orange } \\
\text { pumice; rare felsic phenocrysts, feldspar } \\
\text { and lesser quartz; rare mafic minerals } \\
\text { (biotite, magnetite, clinopyroxene); scarce } \\
\text { lithic fragments. }\end{array}$ & $\begin{array}{c}\text { Tunnel Formation, } \\
\text { Tunnel } 4 \text { Member, } \\
\text { beds. } 4 \mathrm{~J}\end{array}$ \\
\hline $\begin{array}{c}390.1-392.6 \mathrm{~m} \\
(1,280-1,288 \mathrm{ft})\end{array}$ & $\begin{array}{l}\text { Reworked Tuff: Grayish-orange to pale- } \\
\text { yellowish-orange to moderate-reddish- } \\
\text { orange; moderately indurated; zeolitized } \\
\text { to weakly argillized; minor very-pale- } \\
\text { orange pumice; rare felsic phenocrysts; } \\
\text { scarce mafic minerals (biotite); rare lithic } \\
\text { frägments. }\end{array}$ & $\begin{array}{c}\text { Tunnel Formation, } \\
\text { Tunnel } 4 \text { Member, } \\
\text { beds 4FGH, } \\
\text { undifferentiated }\end{array}$ \\
\hline $\begin{array}{c}392.6-401.7 \mathrm{~m} \\
(1,288-1,318 \mathrm{ft})\end{array}$ & $\begin{array}{l}\text { Partially Welded Ash-Flow Tuff: } \\
\text { Mottled grayish-orange and moderate- } \\
\text { reddish-brown; moderately indurated; } \\
\text { zeolitized; minor pale-yellowish-orange } \\
\text { to grayish-yellow pumice to } 8 \mathrm{~mm} \text { in } \\
\text { diameter; rare felsic phenocrysts, } \\
\text { generally feldspar with lesser quartz; rare } \\
\text { mafic minerals (biotite and magnetite); } \\
\text { rare to minor lithic fragments to } 7 \mathrm{~mm} \text { in } \\
\text { diameter. }\end{array}$ & $\begin{array}{l}\text { Tunnel Formation, } \\
\text { Tunnel } 4 \text { Member, } \\
\text { beds } 4 \mathrm{E} \text { (equivalent) }\end{array}$ \\
\hline
\end{tabular}




\begin{tabular}{ccc}
\hline $\begin{array}{c}\text { Depth } \\
\text { Meters/(Feet) }\end{array}$ & Lithologic Description & Stratigraphic Unit \\
\hline
\end{tabular}

401.7-405.4 m Bedded Tuff: Grayish-orange to $(1,318-1,330 \mathrm{ft})$

$405.4-409.7 \mathrm{~m}$ $(1,330-1,344 \mathrm{ft})$

$409.7-414.5 \mathrm{~m}$ $(1,344-1,360 \mathrm{ft})$

$414.5-429.2 \mathrm{~m}$ $(1,360-1,408 \mathrm{ft})$

$429.2-432.2 \mathrm{~m}$ $(1,408-1,418 \mathrm{ft})$ moderate-yellowish-brown; moderately indurated; zeolitized; common grayishorange pumice; common felsic phenocrysts; scarce mafic minerals; rare lithic fragments.

Bedded Tuff: Moderate-reddish-brown; well indurated; zeolitized to weakly argillized; abundant very-pale-orange pumice up to $2 \mathrm{~mm}$ in diameter; minor felsic phenocrysts; rare mafic minerals, rare lithic fragments.

Nonwelded Ash-Flow Tuff: Mottled with grayish-yellow and moderatereddish-orange; moderately indurated; rare grayish-yellow to very-pale-orange pumice; very scarce felsic phenocrysts; very scarce mafic minerals; scarce lithic fragments. Abundant glass shard molds.

Bedded Tuff: Moderate-reddish-brown; well indurated; zeolitized to argillized; minor very-pale-orange pumice; rare to scarce felsic phenocrysts; rare mafic minerals (biotite, magnetite); very scarce lithic fragments.

Bedded Tuff: Dusky-yellow; peralkaline; well indurated; zeolitized; indistinct pumice; common felsic phenocrysts including dipyramidal quartz; scarce magnetite; rare lithic fragments.
Tunnel Formation, Tunnel 4 Member, beds $4 A B C D$, undifferentiated

Tunnel Formation, Tunnel 3 Member, beds $3 \mathrm{D}$
Tunnel Formation, Tunnel 3 Member, beds $3 B C$ (equivalent) ${ }^{2}$

Tunnel Formation, Tunnel 3 Member, beds $3 \mathrm{~A}$

Tub Spring Tuff 


\begin{tabular}{ccc}
\hline $\begin{array}{c}\text { Depth } \\
\text { Meters/(Feet) }\end{array}$ & Lithologic Description & Stratigraphic Unit \\
\hline
\end{tabular}

432.2 - 440.3 m Bedded Tuff: Grayish-yellow; well

tunnel bed 2

$(1,418-1,445 \mathrm{ft})$

$440.3-446.5 \mathrm{~m}$ $(1,445-1,465 \mathrm{ft})$

$446.5-455.7 \mathrm{~m}$ $(1,465-1,495 \mathrm{ft})$

$455.7-492.9 \mathrm{~m}$ $(1,495-1,617 \mathrm{ft})$ indurated; zeolitized; common palegreenish-yellow pumice; common felsic phenocrysts; rare to minor mafic minerals (biotite, magnetite); minor lithic fragments.

Bedded Tuff: Grayish-yellow to palegreenish-yellow, some mottled with grayish-orange-pink; moderately indurated; zeolitized; minor palegreenish-yellow to grayish-orangepink pumice to $1.5 \mathrm{~cm}$ in diameter; minor felsic phenocrysts; minor mafic minerals (magnetite, biotite); very scarce lithic fragments.

Bedded Tuff: Moderate-reddish-brown; well indurated; zeolitized, possibly weakly argillized in some intervals; abundant very-pale-orange pumice up to $2 \mathrm{~mm}$ in diameter; rare felsic phenocrysts; rare mafic minerals and lithic fragments.

Partially Welded Ash-Flow Tuff: Yellowish-gray; well indurated; zeolitized; minor grayish-yellow to pale-greenish-yellow pumice, some with sucrosic secondary mineralization; common felsic phenocrysts of feldspar and quartz, decreasing to minor with depth; common mafic minerals, biotite dominant; minor lithic fragments to $2 \mathrm{~cm}$ in diameter.
Yucca Flat Tuff tunnel bed 2 


\begin{tabular}{ccc}
\hline $\begin{array}{c}\text { Depth } \\
\text { Meters/(Feet) }\end{array}$ & Lithologic Description & Stratigraphic Unit \\
\hline
\end{tabular}

$492.9-506.0 \mathrm{~m}$ $(1,617-1,660 \mathrm{ft})$

$506.0-510.5 \mathrm{~m}$ $(1,660-1,675 \mathrm{ft})$

$510.5-513.6 \mathrm{~m}$ $(1,675-1,685 \mathrm{ft})$

$513.6-516.6 \mathrm{~m}$ $(1,685-1,695 \mathrm{ft})$
Bedded Tuff: Grayish-yellow; well indurated; zeolitized; common to indistinct grayish-yellow pumice to $5 \mathrm{~mm}$ in diameter; felsic phenocrysts variable from rare to abundant; minor mafic minerals, mostly biotite and magnetite; rare lithic fragments.

Bedded Tuff: Dark-reddish-brown; well indurated; zeolitized to weakly argillized; common pale-red pumice exhibiting corroded texture; minor felsic phenocrysts; minor mafic minerals; scarce lithic fragments; sucrosic texture.

Bedded Tuff: Grayish-yellow; well indurated; zeolitized; common to indistinct grayish-yellow pumice; abundant large felsic phenocrysts, including some quartz; common biotite; rare lithic fragments.

Reworked Tuff: Moderate-reddishbrown; well indurated; zeolitized to weakly argillized; common very-paleorange pumice, becoming rare in lower half of interval; abundant felsic phenocrysts; rare mafic minerals of biotite and magnetite; rare small lithic fragments. tunnel bed 1

tunnel bed 1

tunnel bed 1

tunnel bed 1 


\begin{tabular}{ccc}
\hline $\begin{array}{c}\text { Depth } \\
\text { Meters/(Feet) }\end{array}$ & Lithologic Description & Stratigraphic Unit \\
\hline
\end{tabular}

$516.6-522.4 \mathrm{~m}$ $(1,695-1,714 \mathrm{ft})$

$522.4-526.7 \mathrm{~m}$ $(1,714-1,728 \mathrm{ft})$

$526.7-530.4 \mathrm{~m}$ $(1,728-1,740 \mathrm{ft})$

$530.4-538.0 \mathrm{~m}$ $(1,740-1,765 \mathrm{ft})$
Bedded Tuff: Moderate- to darkreddish-brown; poorly to fairly indurated; zeolitized to strongly argillized; scarce to rare pumice; common felsic phenocrysts; rare mafic minerals; :lithic fragments vary from rare to common in reworked zones. Lower contact is probable fault zone.

Bedded Tuff: Grayish-orange-pink to moderate-orange-pink; fair to moderate induration; zeolitized; rare grayish-yellow pumice up to $4 \mathrm{~mm}$ in diameter; rare to minor felsic phenocrysts; rare mafic minerals; rare lithic fragments.

Bedded Tuff: Moderate- to darkreddish-brown; poor to fair induration; zeolitized and argillized; generally common very-pale-orange pumice. Some argillized reworked lithologies with rare pumice; minor felsic phenocrysts; rare mafic minerals and lithic fragments.

Bedded Tuff(?): Grayish-orange, delicately mottled with moderate-reddishbrown; fairly indurated; zeolitized to weakly argillized; minor very-pale-orange pumice; minor felsic phenocrysts; minor mafic minerals (biotite and magnetite); rare lithic fragments. Thin calcite filled fractures. tunnel bed 1

older tuffs, undifferentiated

older tuffs, undifferentiated

older tuffs, undifferentiated 


\begin{tabular}{ccc}
\hline $\begin{array}{c}\text { Depth } \\
\text { Meters/(Feet) }\end{array}$ & Lithologic Description & Stratigraphic Unit \\
\hline
\end{tabular}

$538.0-539.5 \mathrm{~m}$ $(1,765-1,770 \mathrm{ft})$

$539.5-648.9 \mathrm{~m}$ $(1,770-2,129 \mathrm{ft})$

Temporary TD (drill hole)
Paleocolluvim: Moderate to darkreddish-brown: poorly indurated to friable; argillized; tuffaceous; minor grayish-orange-pink pumice; minor felsic phenocrysts; scarce small Paleozoic rock fragments.

Dolostone: Light-olive-gray; fine to medium crystalline; dense; hard; trace vuggy porosity with euhedral dolomite crystals. Some sucrosic texture (on fracture surfaces), trace calcareous; trace moderate-reddish-orange argillaceous fracture fill/coatings. Trace grayish-red to moderate-reddish-brown iron-oxide stain on some fracture surfaces. Moderatereddish-brown finely laminated/fissile clay fracture fill in the $612.6-\mathrm{m}(2,010-\mathrm{ft})$ cuttings sample.

$648.9-680.9 \mathrm{~m}$ $(2,129-2,234 \mathrm{ft})$

$680.9-701.0 \mathrm{~m}$ $(2,234-2,300 \mathrm{ft})$
Dolostone: Light-brownish-gray; cryptocrystalline to medium crystalline; vuggy, with $<5$ percent vuggy porosity. Vugs are $<1 \mathrm{~cm}$ in size and lined in part with euhedral dolomite crystals.

Dolostone: Medium-dark-gray to darkgray; medium crystalline; vuggy in part; scattered nodules and lenses of palereddish-brown, black, and very-paleorange chert; brecciated in part. tuffaceous

Paleocolluvium

Sevy Dolomite

Sevy Dolomite

Laketown Dolomite 


\begin{tabular}{ccc}
\hline $\begin{array}{c}\text { Depth } \\
\text { Meters/(Feet) }\end{array}$ & Lithologic Description & Stratigraphic Unit \\
\hline
\end{tabular}

$701.0-724.2 \mathrm{~m}$ $(2,300-2,376 \mathrm{ft})$

724.2 - 748.9.m $(2,376-2,457 \mathrm{ft})$

$748.9-890.0 \mathrm{~m}$ $(2,457-2,920 \mathrm{ft})$

$890.0-940.6 \mathrm{~m}$ $(2,920-3,086 \mathrm{ft})$
Dolostone: Light-brownish-gray; finely crystalline; vuggy, with $<5$ percent vuggy porosity. Vugs are $<1 \mathrm{~cm}$ in size and lined in part with euhedral dolomite crystals. Brecciated from approximately 712.9 - $713.5 \mathrm{~m}(2,339-2,341 \mathrm{ft})$.

Dolostone: Medium-gray to mediumdark-gray; finely to medium crystalline. Abundant dark-yellowish-orange clay occurs as fracture fillings from approximately $730.6-742.2 \mathrm{~m}(2,397$ $2,435 \mathrm{ft}$ ).

Dolostone: Light-brownish-gray to brownish-gray; finely crystalline; vuggy, with $<5$ percent vuggy porosity. Vugs are less than $1 \mathrm{~cm}$ in size and lined in part with euhedral calcite and dolomite crystals. Fossiliferous from approximately $800.1-809.2 \mathrm{~m}(2,625$ $2,655 \mathrm{ft}$ ) consisting of relic shell fragments. Brecciated in part to $823.0 \mathrm{~m}$ $(2,700 \mathrm{ft})$ with conspicuous moderatereddish-brown clay as fracture fillings.

Dolostone: Medium-gray to medium-

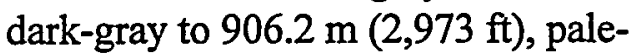
yellowish-brown from $906.2-915.0 \mathrm{~m}$ $(2,973-3,002 \mathrm{ft})$, and medium-dark-gray

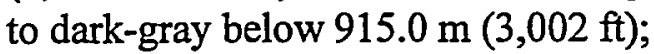
medium crystalline; abundant lenses of pale-yellowish-brown to dark-yellowishbrown chert from $918.4-936.7 \mathrm{~m}$ $(3,013-3,073 \mathrm{ft})$.
Laketown Dolomite

Laketown Dolomite

Laketown Dolomite

Ely Springs Dolomite 


\begin{tabular}{ccc}
\hline $\begin{array}{c}\text { Depth } \\
\text { Meters/(Feet) }\end{array}$ & Lithologic Description & Stratigraphic Unit \\
\hline
\end{tabular}

940.6-952.2 m Sandstone: Light-brownish-gray to

Eureka Quartzite

$(3,086-3,124 \mathrm{ft}) \quad$ brownish-gray, mottled in part; well indurated; 70 percent fine- to mediumgrained, well sorted, subrounded quartz sand; 30 percent dolomite cement becoming mostly silica cemented near base of interval.

$952.2-977.2 \mathrm{~m}$ $(3,124-3,206 \mathrm{ft})$

Final TD

(core hole)
Quartzite: Light-brownish-gray to paleyellowish-brown to $965.9 \mathrm{~m}(3,169 \mathrm{ft})$; very-pale-orange to grayish-orange below; very well indurated; 85 percent medium-grained, very well sorted, subrounded to rounded quartz sand; 15 percent silica cement.

\section{$\underline{\text { Notes }}$}

1 Preliminary work for this site reinforces interpretations based on petrographic work by R. G. Warren (LANL) for the U-3mL site that complex Tw/Ta or Tc relationships exist in this area. Additional work is needed to understand this relationship.

2 Recent studies indicate that the informal unit "Tunnel Formation, Subunit 3BCEquivalent" may be the Tuff of Tolicha Peak (verbal communication, R. G. Warren). 
1. Well ER-6-1 was the second hole drilled for the Groundwater Characterization Project: No completion strings have been installed at this time, but when the well is completed it will be part of the UGTA monitoring well network of the DOE/NV Environmental Restoration Program at the NTS.

2. The 1992 preliminary report for Well ER-6-1 contains data collected through 1 September 1992 (Drellack, et al., 1992). Supplemental data listed below are also extracted from this reference, unless noted otherwise.

3. The lower $328.3 \mathrm{~m}(1,077 \mathrm{ft})$ of Well ER-6-1 was continuously cored in 1994. A draft well-completion report has been prepared that includes data for this phase of drilling (DOE, 1997). This report will be finalized when the completion string(s) have been installed.

4. Approximately $73.2 \mathrm{~m}(240 \mathrm{ft})$ of the stratigraphic section has been faulted out of the lower Tertiary section. This west-dipping fault was intersected at $522.4 \mathrm{~m}(1,714 \mathrm{ft})$.

5. Paleontology samples collected from within the Paleozoic section are available. For further information see Cole and Harris (1996).

6. A fracture analysis report for Well ER-6-1 is available. For further information see IT, 1995.

7. Downhole camera video log for Well ER-6-1 (surface to $460.2 \mathrm{~m}[1,510.0 \mathrm{ft}]$ ) interval is available. $35-\mathrm{mm}$ slides documenting drilling activities, equipment, and samples at Well ER-6-1 are also available.

8. Listings of chloride, bromide, and inorganic carbon content for unwashed drill cuttings samples from the $3.0-125.0 \mathrm{~m}(10-410 \mathrm{ft})$ depth interval are available.

9. Eleven polished thin sections from cuttings samples are available from the interval $146.3-563.9 \mathrm{~m}(480-1,850 \mathrm{ft})$.

10. Water samples were collected during drilling. A fluid level depth of $470.9 \mathrm{~m}(1,545 \mathrm{ft})$ was measured on 09/07/1992.

11. Well ER-6-1 Satellite Hole \#1 is located approximately $16.5 \mathrm{~m}(54 \mathrm{ft})$ south-southeast of Well ER-6-1. Due to the close proximity of this satellite hole to Well ER-6-1, the geology is assumed to be the same. 
Figure 4

West-East Geologic Cross Section Through Well.ER-6-1

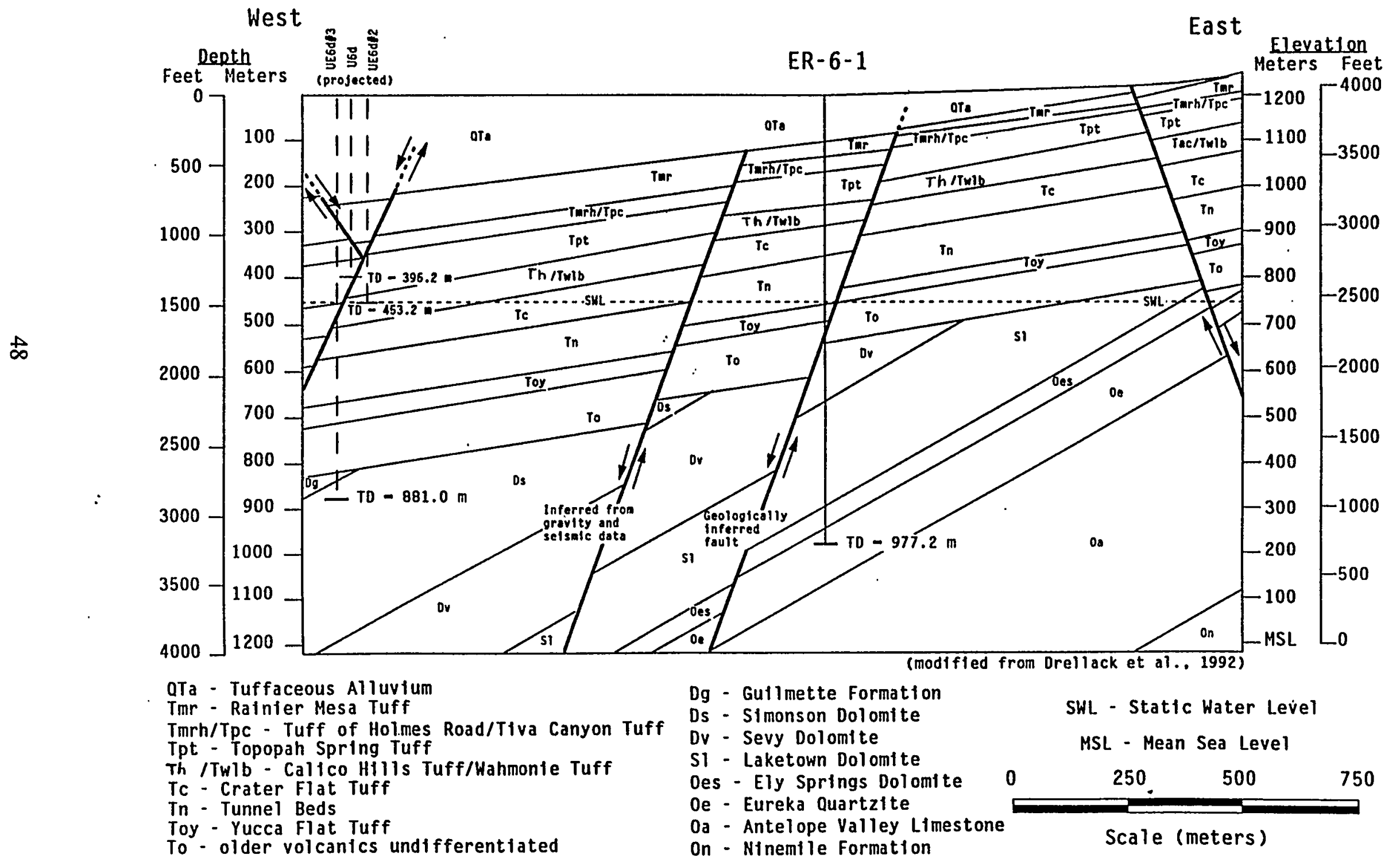




\section{Figure 5}

\section{North South Geologic Cross Section Through Well. ER-6-1}

North

\section{South}

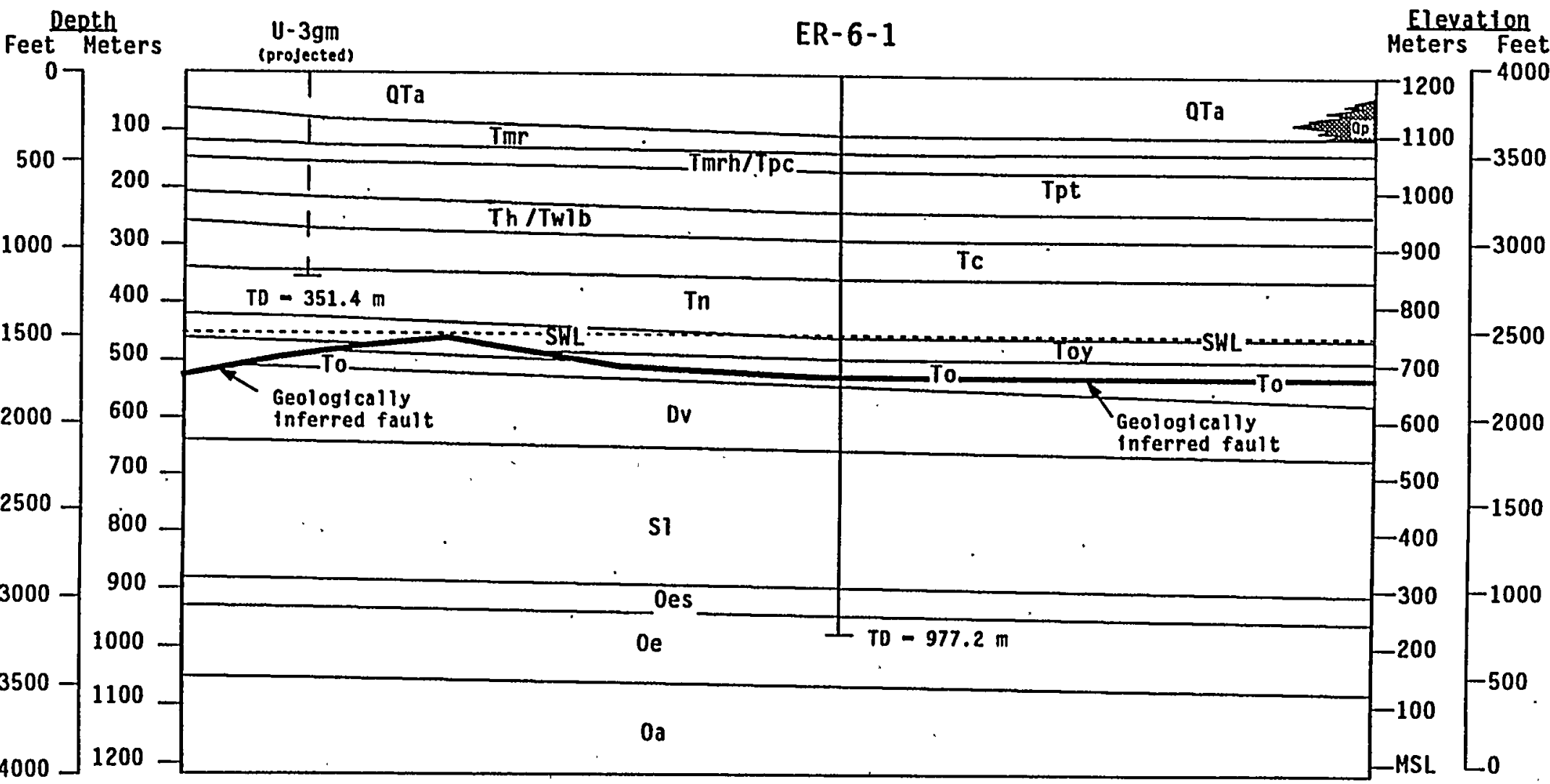

QTa - Tuffaceous Alluvium

Tmr - Rainier Mesa Tuff

(modlfled from Drellack et al.. 1992)

Tmrh/Tpc - Tuff of Holmes Road/TIva Canyon Tuff Tpt - Topopah Spring Tuff

Th /Tw1b - Cal1co Hills Tuff/Wahmonle Tuff

Tc - Crater Flat Tuff

Tn - Tunnel Beds

Toy - Yucca Flat Tuff

To - older volcantcs undifferentiated

Dv - Sevy Dolomite

S1 - Laketown Dolomite

Des - Ely Springs Dolomite

0e - Eureka Quartzite

SWL - Static Water Levei

MSL - Mean Sea Level

0a - Antelope Valley Limestone

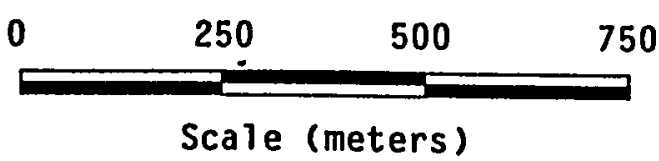


This page intentionally left blank. 
Stratigraphic Log of Emplacement Hole U-7ed

Logged by Robert L. McCall, Raytheon Services Nevada, July 1992

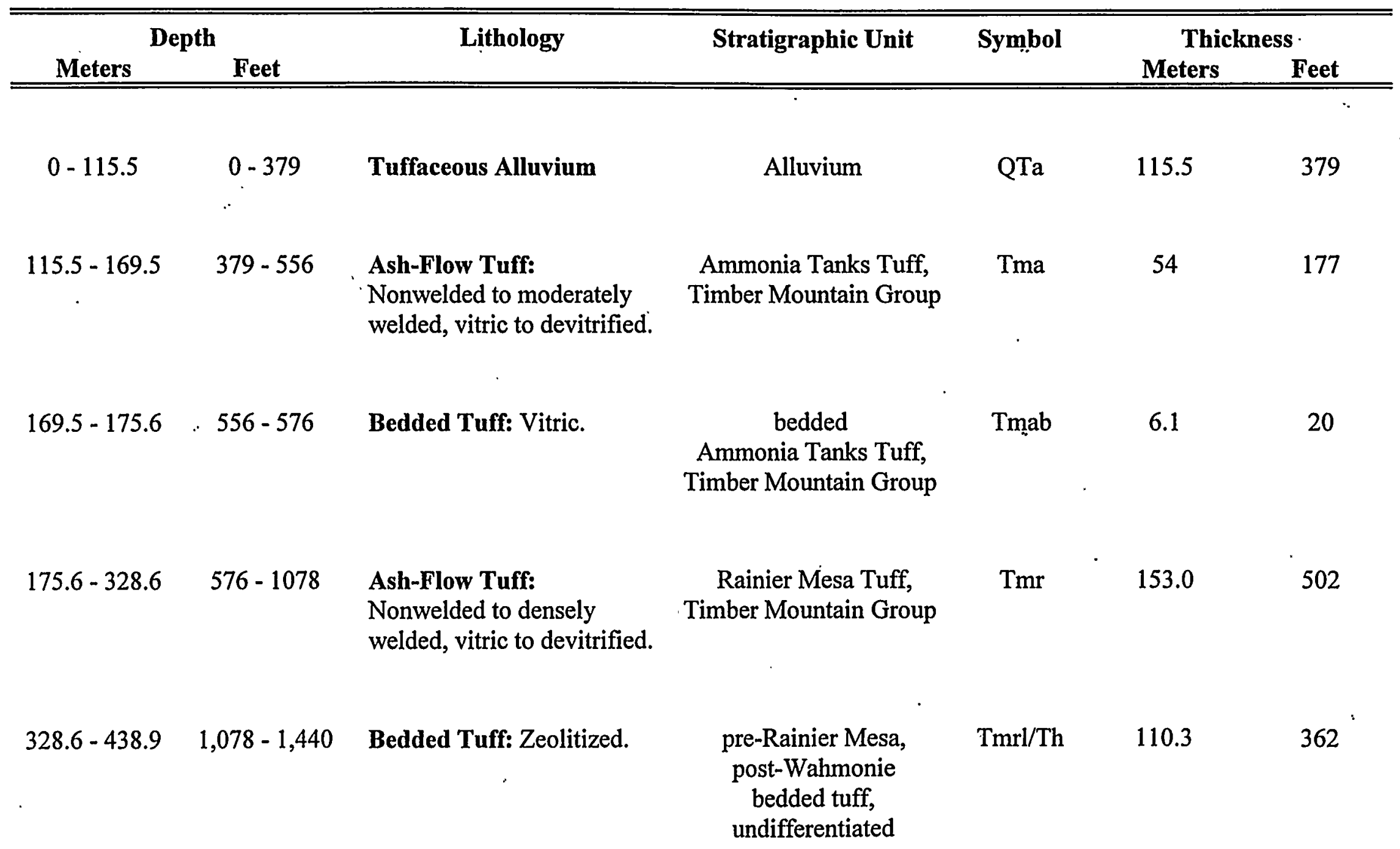


Stratigraphic Log of Emplacement Hole U-7cd (cont.)

\begin{tabular}{|c|c|c|c|c|c|c|}
\hline \multicolumn{2}{|c|}{ Depth } & \multirow[t]{2}{*}{ Lithology } & \multirow[t]{2}{*}{ Stratigraphic Unit } & \multirow[t]{2}{*}{ Symbol } & \multicolumn{2}{|c|}{ Thickness } \\
\hline Meters & Feet & & & & Meters & Feet \\
\hline $438.9-460.2$ & $1,440-1,510$ & Bedded Tuff: Zeolitized. & $\begin{array}{l}\text { tuff of Wahmonie Flat, } \\
\text { Wahmonie Formation }\end{array}$ & Twlb & 21.3 & 70 \\
\hline $\begin{array}{c}460.2-495.3 \\
\text { TD }\end{array}$ & $\begin{array}{c}1,510-1,625 \\
\mathrm{TD}\end{array}$ & Bedded Tuff: Zeolitized. & $\begin{array}{c}\text { Crater Flat Group, } \\
\text { undifferentiated }\end{array}$ & Tc & $>35.1$ & $>115$ \\
\hline
\end{tabular}




\section{Lithologic Log of Emplacement Hole U-7cd}

Logged by Robert L. McCall, Raytheon Services Nevada

July 1992

Unless otherwise noted, the following descriptions refer to washed cuttings samples at 3.05-m (10-ft) intervals. Colors are determined by comparing wet sample color to the Geological Society of America Rock-Color Chart. Stratigraphic contacts and lithologic divisions are tied to geophysical logs whenever possible.

\begin{tabular}{|c|c|c|}
\hline $\begin{array}{c}\text { Depth } \\
\text { Meters/(feet) }\end{array}$ & Lithologic Description & Stratigraphic U \\
\hline $\begin{array}{l}0-36.6 \mathrm{~m} \\
(0-120 \mathrm{ft})\end{array}$ & No samples. & Alluvium \\
\hline $\begin{array}{l}36.6-54.9 \mathrm{~m} \\
(120-180 \mathrm{ft})\end{array}$ & $\begin{array}{l}\text { Tuffaceous Alluvium: Moderate and } \\
\text { dark-yellowish-brown to pale- } \\
\text { yellowish-brown; nonindurated; } \\
\text { subangular to subrounded; moderately } \\
\text { sorted; sand to. gravel sizes; tuffaceous } \\
\text { with } 5 \text { to } 10 \text { percent Paleozoic rock } \\
\text { fragments; weakly calcareous. }\end{array}$ & Alluvium \\
\hline $\begin{array}{l}54.9-76.2 \mathrm{~m} \\
(180-250 \mathrm{ft})\end{array}$ & $\begin{array}{l}\text { Tuffaceous Alluvium: Samples are } \\
\text { varicolored; poorly indurated; } \\
\text { subrounded; poorly sorted; } \\
\text { conspicuous increase of pebble-sized } \\
\text { tuffaceous fragments with } 10 \text { percent } \\
\text { Paleozoic rock fragments of which } \\
\text { more than } 5 \text { percent are carbonate; } \\
\text { moderately calcareous; carbonate-rich } \\
\text { pebble bed from } 73.2 \text { - } 76.2 \mathrm{~m} \text { ( } 240 \text { - } \\
250 \mathrm{ft}) \text {. }\end{array}$ & Alluvium \\
\hline $\begin{array}{c}76.2-115.5 \mathrm{~m} \\
(250-379 \mathrm{ft})\end{array}$ & $\begin{array}{l}\text { Tuffaceous Alluvium: Grayish- } \\
\text { orange to moderately-yellowish-brown; } \\
\text { nonindurated; well sorted; subangular } \\
\text { to subrounded; fine to very coarse sand } \\
\text { sizes; tuffaceous alluvium with } \\
5 \text { percent Paleozoic rock fragments; } \\
\text { calcareous; increasing to } 10 \text { percent } \\
\text { Paleozoic rock fragments (<2 percent } \\
\text { are carbonate) below } 106.7 \mathrm{~m} \text { ( } 350 \mathrm{ft}) \text {. }\end{array}$ & Alluvium \\
\hline
\end{tabular}




\begin{tabular}{|c|c|c|}
\hline $\begin{array}{c}\text { Depth } \\
\text { Meters/(feet) }\end{array}$ & Lithologic Description & Stratigraphic Unit \\
\hline $\begin{array}{c}115.5-133.8 \mathrm{~m} \\
(379-439 \mathrm{ft})\end{array}$ & $\begin{array}{l}\text { Nonwelded Ash-Flow Tuff: } \\
\text { Moderate-yellowish-brown to grayish- } \\
\text { orange; vitric; poorly indurated; minor } \\
\text { pinkish-gray to dark-yellowish-gray } \\
\text { pumice; minor felsic phenocrysts of } \\
\text { quartz and feldspar; rare mafic } \\
\text { minerals of biotite and magnetite; } \\
\text { scarce dark-reddish-brown lithic } \\
\text { fragments. }\end{array}$ & Ammonia Tanks Tuff \\
\hline $\begin{array}{c}133.8-139.0 \mathrm{~m} \\
(439-456 \mathrm{ft})\end{array}$ & $\begin{array}{l}\text { Partially Welded Ash-Flow Tuff: } \\
\text { Grayish-orange; partially devitrified; } \\
\text { minor felsic phenocrysts of quartz, } \\
\text { chatoyant sanidine, and other feldspars; } \\
\text { rare mafic minerals of biotite and } \\
\text { magnetite; scarce dark-reddish-brown } \\
\text { lithic fragments up to } 5 \text { mm in } \\
\text { diameter; common black cuspate glass } \\
\text { shards. }\end{array}$ & Ammonia Tanks Tuff \\
\hline $\begin{array}{c}139.0-149.4 \mathrm{~m} \\
(456-490 \mathrm{ft})\end{array}$ & $\begin{array}{l}\text { Nonwelded Ash-Flow Tuff: Grayish- } \\
\text { orange becoming pale-brown below } \\
146.3 \mathrm{~m} \text { ( } 480 \mathrm{ft} \text { ); vitric to partially } \\
\text { devitrified; poorly indurated; minor } \\
\text { light-gray pumice; common felsic } \\
\text { phenocrysts of chatoyant sanidine, } \\
\text { quartz, and lesser amounts of other } \\
\text { feldspars; rare mafic minerals of biotite } \\
\text { and magnetite; scarce grayish-red- } \\
\text { purple lithic fragments up to } 5 \mathrm{~mm} \text { in } \\
\text { diameter; minor black cuspate glass } \\
\text { shards. }\end{array}$ & Ammonia Tanks Tuff \\
\hline
\end{tabular}




\begin{tabular}{|c|c|c|}
\hline $\begin{array}{c}\text { Depth } \\
\text { Meters/(feet) }\end{array}$ & Lithologic Description & Stratigraphic Unit \\
\hline $\begin{array}{c}149.4-169.5 \mathrm{~m} \\
(490-556 \mathrm{ft})\end{array}$ & $\begin{array}{l}\text { Nonwelded Ash-Flow Tuff: Pale- } \\
\text { yellowish-brown; sample is enriched in } \\
\text { felsic phenocrysts; vitric; poorly } \\
\text { indurated; common chatoyant sanidine, } \\
\text { quartz and feldspars; scarce biotite, } \\
\text { magnetite and clinopyroxene; scarce } \\
\text { lithic fragments; trace of grayish- } \\
\text { orange glass shards. }\end{array}$ & Ammonia Tanks Tuff \\
\hline $\begin{array}{c}169.5-175.6 \mathrm{~m} \\
(556-576 \mathrm{ft})\end{array}$ & $\begin{array}{l}\text { Bedded Tuff: Very-pale-orange; } \\
\text { poorly indurated; vitric; minor felsic } \\
\text { phenocrysts of quartz and feldspar; } \\
\text { scarce mafic minerals of biotite and } \\
\text { magnetite; scarce lithic fragments. }\end{array}$ & $\begin{array}{c}\text { bedded } \\
\text { Ammonia Tanks Tuff }\end{array}$ \\
\hline $\begin{array}{c}175.6-203.0 \mathrm{~m} \\
(576-666 \mathrm{ft})\end{array}$ & $\begin{array}{l}\text { Nonwelded Ash-Flow Tuff: Grayish- } \\
\text { orange becoming dark-yellowish- } \\
\text { brown to pale-brown below } 179.8 \mathrm{~m} \\
(590 \mathrm{ft}) \text {; poorly indurated; vitric } \\
\text { becoming partially devitrified below } \\
179.8 \mathrm{~m}(590 \mathrm{ft}) \text {; minor light-brown } \\
\text { pumice; felsic phenocrysts of quartz } \\
\text { and feldspar; scarce mafic minerals of } \\
\text { biotite, hornblende, and magnetite; } \\
\text { scarce lithics; "crystal slurry" from } \\
173.7 \text { - } 192.0 \mathrm{~m} \text { ( } 570 \text { - } 630 \mathrm{ft}) \text {. }\end{array}$ & $\begin{array}{c}\text { mafic-rich } \\
\text { Rainier Mesa Tuff }\end{array}$ \\
\hline $\begin{array}{c}203.0-211.8 \mathrm{~m} \\
(666-695 \mathrm{ft})\end{array}$ & $\begin{array}{l}\text { Partially Welded Ash-Flow Tuff: } \\
\text { Moderate-yellowish-brown; } \\
\text { moderately indurated; devitrified; } \\
\text { minor moderate-reddish-brown } \\
\text { pumice; minor felsic phenocrysts of } \\
\text { dipyramidal quartz and feldspar; rare } \\
\text { mafic minerals of biotite, hornblende, } \\
\text { clinopyroxene and magnetite. }\end{array}$ & $\begin{array}{c}\text { mafic-rich } \\
\text { Rainier Mesa Tuff }\end{array}$ \\
\hline
\end{tabular}


Lithologic Log of Emplacement Hole U-7cd (cont.)

\begin{tabular}{|c|c|c|}
\hline $\begin{array}{c}\text { Depth } \\
\text { Meters/(feet) }\end{array}$ & Lithologic Description & Stratigraphic Unit \\
\hline $\begin{array}{c}211.8-214.3 \mathrm{~m} \\
(695-703 \mathrm{ft})\end{array}$ & $\begin{array}{l}\text { Moderately Welded Ash-Flow Tuff: } \\
\text { Light-brown (5YR5/6) to moderate- } \\
\text { yellowish-brown; moderate induration; } \\
\text { devitrified; minor varicolored pumice } \\
\text { with vapor-phase mineralization } \\
\text { present; minor felsic phenocrysts of } \\
\text { dipyramidal quartz and feldspar; rare } \\
\text { mafic minerals of biotite, } \\
\text { clinopyroxene, and magnetite; trace of } \\
\text { lithic fragments. }\end{array}$ & $\begin{array}{c}\text { mafic-rich } \\
\text { Rainier Mesa Tuff }\end{array}$ \\
\hline $\begin{array}{c}214.3-217.6 \mathrm{~m} \\
(703-714 \mathrm{ft})\end{array}$ & $\begin{array}{l}\text { Densely Welded Ash-Flow Tuff: } \\
\text { Moderate-brown (5YR4/4); well } \\
\text { indurated; partially devitrified with } \\
\text { some silicification; minor felsic } \\
\text { phenocrysts of quartz and feldspar; rare } \\
\text { mafic minerals of biotite, } \\
\text { clinopyroxene, and magnetite; trace of } \\
\text { lithic fragments; minor pieces of black } \\
\text { vitrophyre in cuttings samples. }\end{array}$ & $\begin{array}{c}\text { mafic-rich } \\
\text { Rainier Mesa Tuff }\end{array}$ \\
\hline $\begin{array}{c}217.6-230.7 \mathrm{~m} \\
(714-757 \mathrm{ft})\end{array}$ & $\begin{array}{l}\text { Moderately Welded Ash-Flow Tuff: } \\
\text { Light-brown (5YR6/4) to moderate- } \\
\text { brown (5YR4/4); moderately } \\
\text { indurated; devitrified; minor to rare } \\
\text { felsic phenocrysts of dipyramidal } \\
\text { quartz and feldspar; trace of mafic } \\
\text { minerals of biotite and magnetite; trace } \\
\text { of dark-reddish-brown lithic fragments. }\end{array}$ & $\begin{array}{c}\text { mafic-rich } \\
\text { Rainier Mesa Tuff }\end{array}$ \\
\hline
\end{tabular}




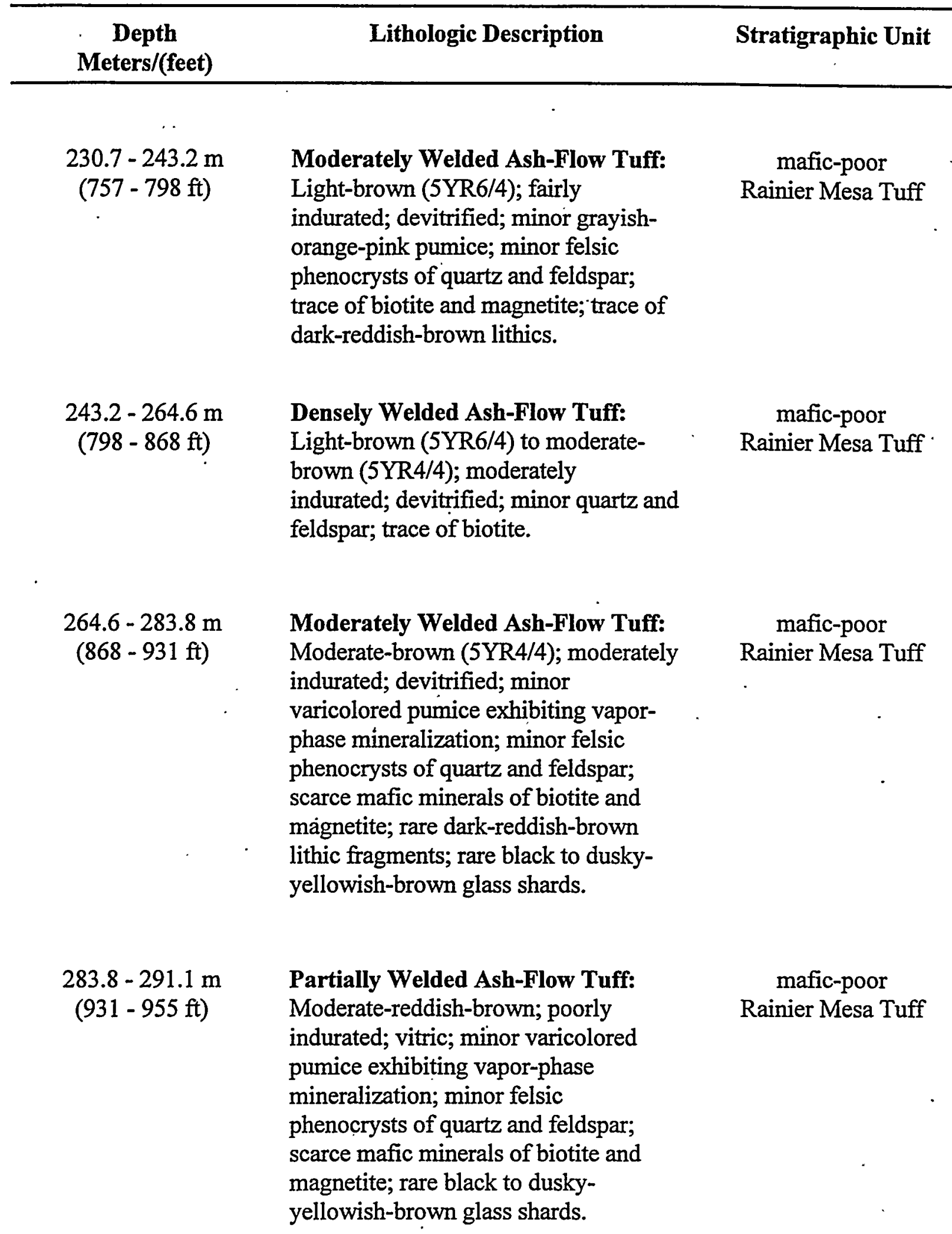


Lithologic Log of Emplacement Hole U-7cd (cont.)

\begin{tabular}{|c|c|c|}
\hline $\begin{array}{c}\text { Depth } \\
\text { Meters/(feet) }\end{array}$ & Lithologic Description & Stratigraphic Unit \\
\hline $\begin{array}{l}291.1-317.0 \mathrm{~m} \\
(955-1,040 \mathrm{ft})\end{array}$ & $\begin{array}{l}\text { Nonwelded Ash-Flow Tuff: Pale-red } \\
\text { (10R6/2); ("crystal slurry") friable; } \\
\text { vitric; very-light-gray pumice; felsic } \\
\text { phenocrysts of quartz and feldspar; } \\
\text { trace of mafic minerals of biotite and } \\
\text { magnetite; minor varicolored lithic } \\
\text { fragments. }\end{array}$ & $\begin{array}{c}\text { mafic-poor } \\
\text { Rainier Mesa Tuff }\end{array}$ \\
\hline $\begin{array}{c}317.0-328.6 \mathrm{~m} \\
(1,040-1,078 \mathrm{ft})\end{array}$ & $\begin{array}{l}\text { Nonwelded Ash-Flow Tuff: Very-. } \\
\text { pale-orange to grayish-orange; fairly } \\
\text { indurated; weakly zeolitized; minor } \\
\text { very small grayish-orange pumice; } \\
\text { minor felsic phenocrysts of quartz and } \\
\text { feldspars; trace mafic minerals of } \\
\text { biötite, hornblende and magnetite; } \\
\text { scarce lithic fragments. }\end{array}$ & $\begin{array}{c}\text { mafic-poor } \\
\text { Rainier Mesa Tuff }\end{array}$ \\
\hline $\begin{array}{c}328.6-341.4 \mathrm{~m} \\
(1,078-1,120 \mathrm{ft})\end{array}$ & $\begin{array}{l}\text { Bedded Tuff: Very-pale-orange to } \\
\text { grayish-orange; fairly indurated; } \\
\text { weakly zeolitized; minor felsic } \\
\text { phenocrysts of quartz and feldspars; } \\
\text { trace of biotite and magnetite; scarce } \\
\text { lithic fragments; minor clear glass } \\
\text { shards; "crystal slurry" from } 335.3 \text { - } \\
341.4 \mathrm{~m}(1,100-1,120 \mathrm{ft}) \text {. }\end{array}$ & $\begin{array}{l}\text { pre-Rainier Mesa, } \\
\text { post-Wahmonie } \\
\text { bedded tuff, } \\
\text { undifferentiated }\end{array}$ \\
\hline $\begin{array}{c}341.4-359.7 \mathrm{~m} \\
(1,120-1,180 \mathrm{ft})\end{array}$ & $\begin{array}{l}\text { Bedded Tuff: Very-pale-orange to } \\
\text { grayish-orange; fairly indurated; } \\
\text { zeolitized; minor felsic phenocrysts of } \\
\text { quartz and feldspars; trace of biotite } \\
\text { and magnetite; scarce lithic fragments. }\end{array}$ & $\begin{array}{l}\text { pre-Rainier Mesa, } \\
\text { post-Wahmonie } \\
\text { bedded tuff, } \\
\text { undifferentiated }\end{array}$ \\
\hline
\end{tabular}


Lithologic Log of Emplacement Hole U-7cd (cont.)

Depth

Meters/(feet)
Lithologic Description

Bedded Tuff: Grayish-orange to moderate-yellowish-brown; moderately indurated; zeolitized; minor very-paleorange pumice; minor felsic phenocrysts of quartz and feldspar; trace mafic minerals of biotite, magnetite and hornblende; minor dusky-brown lithic fragments up to $5 \mathrm{~mm}$ in diameter.

$393.2-402.3 \mathrm{~m}$ $(1,290-1,320 \mathrm{ft})$

$402.3-414.5 \mathrm{~m}$ $(1,320-1,360 \mathrm{ft})$

$414.5-417.6 \mathrm{~m}$ $(1,360-1,370 \mathrm{ft})$ indurated; zeolitized; common feldspars with scarce quartz; sample enriched in felsics and lithics from $396.2-402.3 \mathrm{~m}(1,300-1,320 \mathrm{ft})$; rare mafic minerals of biotite; common grayish-red (10R4/2) lithic fragments up to $3 \mathrm{~mm}$ in diameter.

Bedded Tuff: Grayish-orange; zeolitized; poorly indurated; common very-pale-orange pumice; minor felsic phenocrysts of quartz and feldspar; scarce mafic minerals of biotite; scarce grayish-red (10R4/2) lithic fragments.

Air-Fall Tuff: Grayish-yellow; zeolitized; poorly indurated; minor felsic phenocrysts of quartz and feldspar; scarce mafic minerals of biotite; scarce lithic fragments.
Bedded Tuff: Very-pale-orange; fairly
Stratigraphic Unit

pre-Rainier Mesa, post-Wahmonie bedded tuff, undifferentiated
pre-Rainier Mesa, post-Wahmonie bedded tuff, undifferentiated

Calico Hills Formation

Calico Hills Formation 
Lithologic Log of Emplacement Hole U-7cd (cont.)

\begin{tabular}{|c|c|c|}
\hline $\begin{array}{c}\text { Depth } \\
\text { Meters/(feet) }\end{array}$ & Lithologic Description & Stratigraphic Unit \\
\hline $\begin{array}{c}417.6-438.9 \mathrm{~m} \\
\left(1,370-1,440^{\circ} \mathrm{ft}\right)\end{array}$ & $\begin{array}{l}\text { Bedded Tuff: Very-pale-orange; } \\
\text { zeolitized; moderately indurated; } \\
\text { common very-light-gray pumice; rare } \\
\text { felsic phenocrysts of quartz and } \\
\text { feldspar; scarce mafic minerals of } \\
\text { biotite; rare dark-reddish-brown lithic } \\
\text { fragments up to } 1 \mathrm{~mm} \text { in diameter. }\end{array}$ & Calico Hills Formatio \\
\hline $\begin{array}{c}438.9-460.2 \mathrm{~m} \\
(1,440-1,510 \mathrm{ft})\end{array}$ & $\begin{array}{l}\text { Bedded Tuff: Grayish-orange to dark- } \\
\text { yellowish-brown; zeolitized; poorly to } \\
\text { moderately indurated; white to light- } \\
\text { gray pumice; common felsic } \\
\text { phenocrysts of feldspar; scarce mafic } \\
\text { minerals of biotite, hornblende and } \\
\text { magnetite; scarce light-brown } \\
\text { (5YR5/6) lithics. }\end{array}$ & tuff of Wahmonie Fla \\
\hline $\begin{array}{c}460.2-495.3 \mathrm{~m} \\
(1,510-1,625 \mathrm{ft}) \\
\text { TD }\end{array}$ & $\begin{array}{l}\text { Bedded Tuff: Grayish-orange; } \\
\text { zeolitized; moderately indurated; very- } \\
\text { pale-orange pumice; common felsic } \\
\text { phenocrysts of feldspar and quartz; rare } \\
\text { mafic-minerals of biotite, hornblende } \\
\text { and magnetite; common dusky-brown } \\
\text { lithic fragments; minor mottling with } \\
\text { moderate-reddish-orange color at } \\
478.5 \mathrm{~m}(1,570 \mathrm{ft}) \text {; air-fall with large } \\
\text { felsic phenocrysts from } 484.6 \text { - } \\
490.7 \mathrm{~m}(1,590-1,610 \mathrm{ft}) \text {. }\end{array}$ & $\begin{array}{l}\text { Crater Flat Group } \\
\text { (undifferentiated) }\end{array}$ \\
\hline
\end{tabular}




\section{Emplacement Hole U-7ed Supplemental Data}

1. For additional information regarding Emplacement Hole U-7cd, see McCall (1993). Supplemental data listed below are also extracted from McCall (1993) except as noted.

2. The equilibrated water level as measured in open boreholes in the U-7cd/U-7cd Sat \#1 area is higher than had been predicted from older drill hole data. This is thought to be due to the presence of a pressurized aquifer resulting from nearby nuclear tests. Pre-drill estimate of depth to water level at U-7cd was $515.1 \mathrm{~m}(1,690 \mathrm{ft})$. However, fluid-level ${ }^{\text {. }}$ measurements in the U-7cd borehole on 02/16/1993 recorded a depth to fluid of $474.9 \mathrm{~m}$ $(1,558 \mathrm{ft})$.

3. The latest water-level tag in U-7cd, made on $4 / 9 / 1997$, indicated a depth to fluid of $438.9 \pm 0.9 \mathrm{~m}(1,440 \pm 3 \mathrm{ft})$. The original pump installed in the hole was found to be inoperable at that time, and estimated to be buried by approximately $12.2 \mathrm{~m}(40 \mathrm{ft})$ of fill at the bottom of the hole (BN, 1997). A bottom-hole tag was made at $475 \pm 0.9 \mathrm{~m}$ $(1,559 \pm 3 \mathrm{ft})$ on $4 / 9 / 1997$.

4. U-7cd Sat \#1 is located approximately $9 \mathrm{~m}(29.5 \mathrm{ft})$ north-northeast of U-7cd.

5. See graphic data summary sheet in back pocket. 


\section{Figure 6}

West-East Geologic Cross Section Through U-7cd

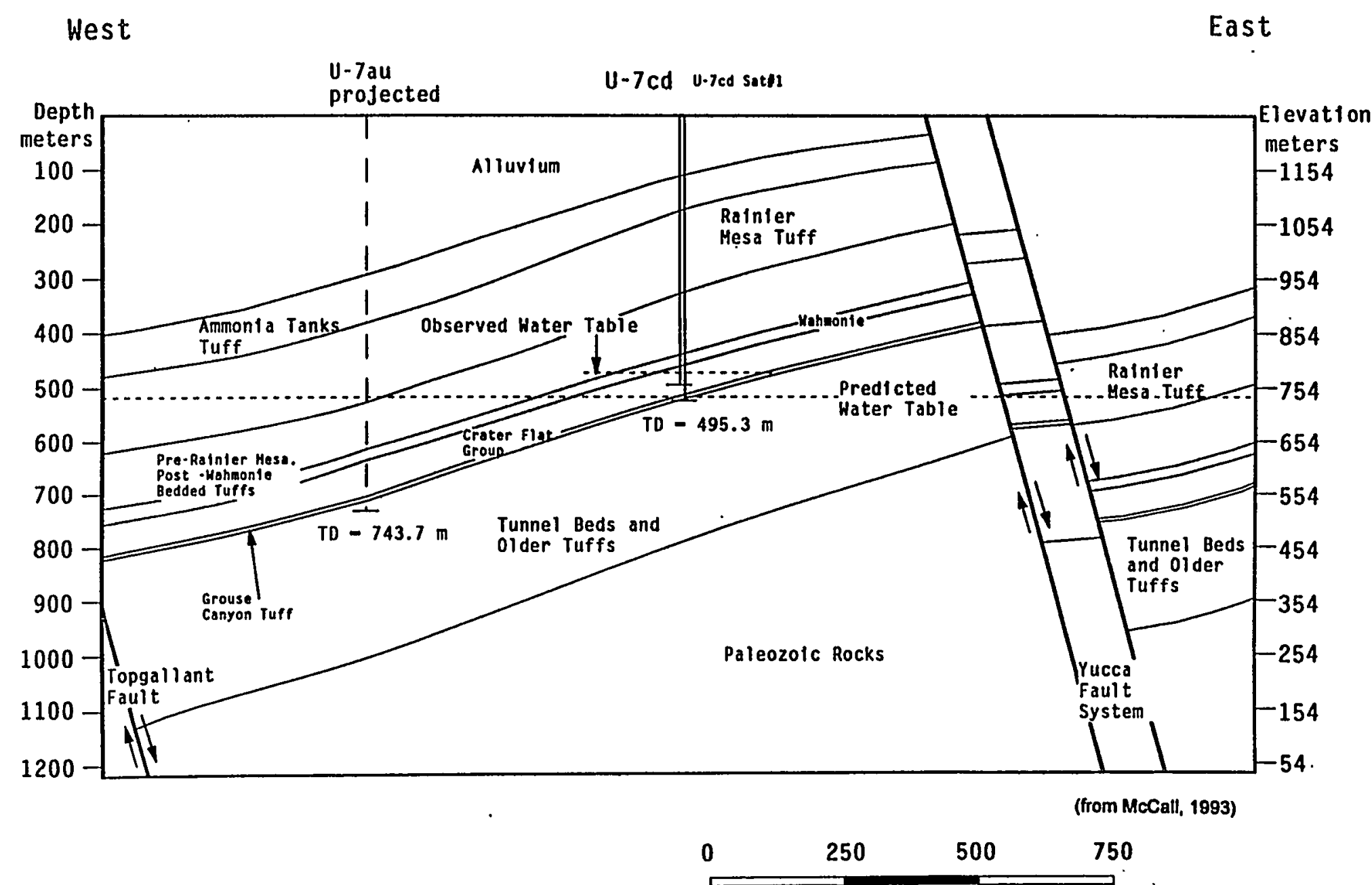

Scale (meters) 
Figure 7

North-South Geologic Cross Section Through U-7cd

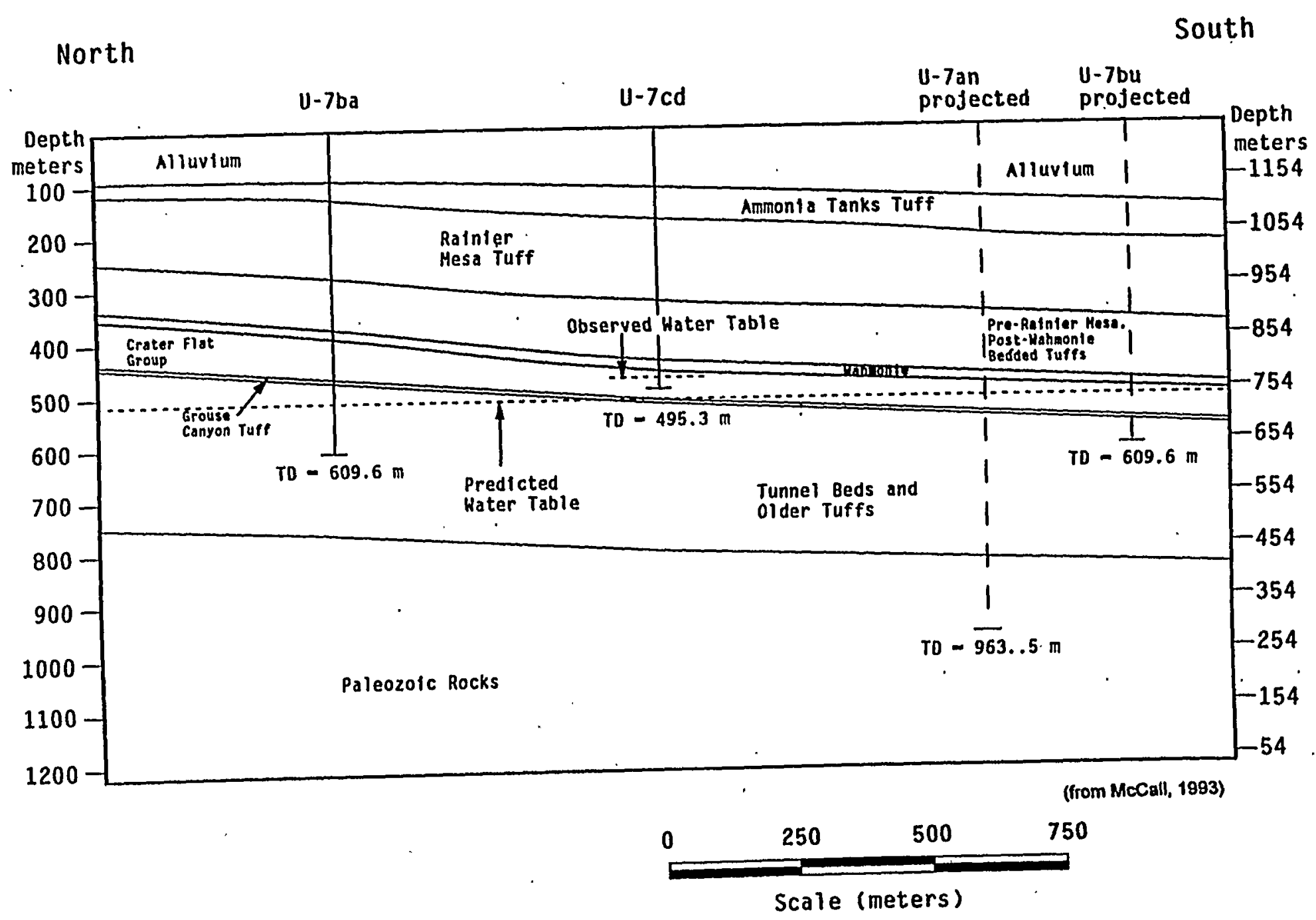


This page intentionally left blank. 
Stratigraphic Log for U-7ed Satellite Hole \#1

Logged by Robert L. McCall, Raytheon Services Nevada, July 1992

\begin{tabular}{|c|c|c|c|c|c|c|}
\hline \multicolumn{2}{|c|}{ Depth } & \multirow[t]{2}{*}{ Lithology } & \multirow[t]{2}{*}{ Stratigraphic Unit } & \multirow[t]{2}{*}{ Symbol } & \multicolumn{2}{|c|}{ Thickness } \\
\hline Meters & Feet & & & & Meters & Feet \\
\hline $0-110.6$ & $0-363$ & Tuffaceous Alluvium. & Alluvium & QTa & 110.6 & 363 \\
\hline $110.6-166.1$ & $363-545$ & $\begin{array}{l}\text { Ash-Flow Tuff: } \\
\text { Nonwelded to moderately } \\
\text { welded, vitric to devitrified. }\end{array}$ & $\begin{array}{l}\text { Ammonia Tanks Tuff, } \\
\text { Timber Mountain Group }\end{array}$ & Tma & 55.5 & 182 \\
\hline $166.1-171.6$ & $545-563$ & Bedded Tuff: Vitric. & $\begin{array}{c}\text { bedded } \\
\text { Ammonia Tanks Tuff, } \\
\text { Timber Mountain Group }\end{array}$ & Tmab & 5.5 & 18 \\
\hline $171.6-327.4$ & $563-1074$ & $\begin{array}{l}\text { Ash-Flow Tuff: } \\
\text { Nonwelded to densely } \\
\text { welded, vitric to devitrified. }\end{array}$ & $\begin{array}{l}\text { Rainier Mesa Tuff, } \\
\text { Timber Mountain Group }\end{array}$ & $\operatorname{Tmr}$ & 155.8 & 511 \\
\hline $327.4-434.9$ & $1,074-1,427$ & Bedded Tuff: Zeolitized. & $\begin{array}{l}\text { pre-Rainier Mesa, } \\
\text { post-Wahmonie } \\
\text { bedded tuff, } \\
\text { undifferentiated }\end{array}$ & $\mathrm{Tmrl} / \mathrm{Th}$ & 107.5 & 353 \\
\hline
\end{tabular}


Stratigraphic Log of U-7cd Satellite Hole \#1 (cont.)

\begin{tabular}{|c|c|c|c|c|c|c|}
\hline \multicolumn{2}{|c|}{ Depth } & \multirow[t]{2}{*}{ Lithology } & \multirow[t]{2}{*}{ Stratigraphic Unit } & \multirow[t]{2}{*}{ Symbol } & \multicolumn{2}{|c|}{ Thickness } \\
\hline Meters & Feet & & & & Meters & Feet \\
\hline $434.9-457.5$ & $1,427-1,501$ & Bedded Tuff: Zeolitized. & tuff of Wahmonie Flat & Twlb & 22.6 & 74 \\
\hline $457.5-505.4$ & $1,501-1,658$ & Bedded Tuff: Zeolitized. & $\begin{array}{l}\text { Crater Flat Group, } \\
\text { undifferentiated }\end{array}$ & $\mathrm{Tc}$ & 47.9 & 157 \\
\hline $505.4-511.5$ & $1,658-1,678$ & Air-Fall Tuff: Zeolitized. & $\begin{array}{l}\text { bedded } \\
\text { Grouse Canyon Tuff, } \\
\text { Belted Range Group }\end{array}$ & Tbgb & 6.1 & 20 \\
\hline
\end{tabular}




\section{U-7cd Satellite Hole \#1 Supplemental Data}

1. For additional information regarding this site, see McCall (1993). The supplemental data listed below are also extracted from McCall (1993).

2. U-7cd Sat \#1 is located approximately $9 \mathrm{~m}(29.5 \mathrm{ft})$ north-northeast of U-7cd. The satellite hole was used to help define the geology and provide drilling-related data useful in evaluation of this site for containment purposes.

3. The equilibrated water level as measured in open boreholes in the U-7cd/U-7cd Sat \#1 area is higher than had been predicted from older drill hole data. The higher water level observed is believed to be the potentiometric level of a pressurized aquifer resulting from nearby nuclear tests.

4. Water level measurements at U-7cd Sat \#1 recorded a depth to fluid level of $433.1 \mathrm{~m}$ $(1,421 \mathrm{ft})$ on $01 / 29 / 1993$. 
This page intentionally left blank. 
Emplacement Hole U-19bf was abandoned in January 1990 due to severe sloughing while at a temporary total depth of $471.2 \mathrm{~m}$ (1,546 $\mathrm{ft}$ ). A bottom hole measurement on July 9 , 1992 indicated a total depth of $155.4 \mathrm{~m}(510 \mathrm{ft})$. The geology provided below is based on the following information: (1) results of examination of unwashed U-19bf drill cuttings samples from 18.3 - $402.3 \mathrm{~m}$ (60 - 1,320 ft) (Rayburn, 1990); (2) geology encountered in emplacement hole U-19ak reported in Ferguson et al. (1994); and (3) surface geology depicted in Noble et al. (1967). Because no geophysical logs were run at U-19bf, the stratigraphic contacts listed below are approximate: Stratigraphic nomenclature is from Ferguson et al. (1994).

\begin{tabular}{lllllll}
\hline \hline Depth & & Lithology & $*$ & Stratigraphic Unit & Symbol & \multicolumn{2}{c}{ Thickness } \\
Meters & Feet & & & Meters & Feet \\
\hline \hline
\end{tabular}

\section{$0-36.6 \quad 0-120 \quad$ Ash-Flow Tuff:}

Nonwelded to partially welded, vitric to devitrified.

Bedded Tuff: Vitric.

\section{Ash-Flow Tuff:} Nonwelded to densely welded.

$149.4-292.6 \quad 490-960$

$292.6-471.2$

TD
$140-490$

$960-1,546$

TD

\section{Bedded Tuff: Very \\ friable, sloughing zone.}

Lava: Vitric to

approximately $341.4 \mathrm{~m}$ $(1,120 \mathrm{ft})$, devitrified below.
Ammonia Tanks Tuff,

Tma

36.6

120

Timber Mountain Group

bedded

Tmab

6.1

Ammonia Tanks Tuff, Timber Mountain Group

\section{Rainier Mesa Tuff,} Timber Mountain Group

$\mathrm{Tmr}$

106.7

Calico Hills Formation,

Dead Horse Flat Formation, Volcanics of Area 20 Tbd $>178.6$ $>586$

Tac

143.2 Belted Range Group 


\section{Emplacement Hole U-19bf Supplemental Data}

1. Emplacement Hole U-19bf was abandoned in January 1990 due to severe sloughing while at a temporary total depth of $471.2 \mathrm{~m}$ (1546 ft). A bottom hole measurement on 9 July 1992 indicated a total depth of $155.4 \mathrm{~m}(510 \mathrm{ft})$ with no fluid.

2. Borehole erosion occurred from 90.5 - $116.1 \mathrm{~m}(297-381 \mathrm{ft})$ with a large diameter (6.1 $9.1 \mathrm{~m}[20-30 \mathrm{ft}])$ sloughed section below $148.7 \mathrm{~m}(488 \mathrm{ft})$. Vertical fractures are present from 70.1 - $76.8 \mathrm{~m}(230$ - $252 \mathrm{ft})$ (RSN, 1991).

3. Sandia National Laboratory color video, used to observe and measure caving intervals, is availàble (Rayburn, 1989).

4. No geophysical logs were run at U-19bf. 
Stratigraphic Log of Emplacement Hole U-19bg

Logged by Lance B. Prothro, Raytheon Services Nevada, August 1991

\begin{tabular}{|c|c|c|c|c|c|c|}
\hline \multirow[b]{2}{*}{ Meters } & Depth & \multirow[t]{2}{*}{ Lithology } & \multirow[t]{2}{*}{ Stratigraphic Unit } & \multirow[t]{2}{*}{ Symbol } & \multicolumn{2}{|c|}{ Thickness } \\
\hline & Feet & & & & Meters & Feet \\
\hline $0-9.1$ & $0-30$ & No samples. & $\begin{array}{c}\text { Thirsty Canyon Group, } \\
\text { undifferentiated }\end{array}$ & $\mathrm{Tt}$ & 9.1 & 30 \\
\hline $9.1-29.0$ & $30-95.3$ & $\begin{array}{l}\text { Ash-Flow Tuff: Partially } \\
\text { welded, devitrified. }\end{array}$ & $\begin{array}{l}\text { Pahute Mesa Tuff, } \\
\text { Thirsty Canyon Group }\end{array}$ & Ttp & 19.9 & 65.3 \\
\hline $29.0-36.0$ & $95.3-118$ & $\begin{array}{l}\text { Bedded Tuff: Vitric, partly } \\
\text { zeolitic, calcareous. }\end{array}$ & $\begin{array}{c}\text { rhyolite of } \\
\text { Chukar Canyon, } \\
\text { Volcanics of Fortymile } \\
\text { Canyon }\end{array}$ & Tffbr & 7.0 & 22.7 \\
\hline $36.0-48.9$ & $118-160.5$ & Bedded Tuff: Vitric. & $\begin{array}{c}\text { rhyolite of } \\
\text { Beatty Wash, } \\
\text { Volcanics of Fortymile } \\
\text { Canyọn }\end{array}$ & Tfbw & 12.9 & 42.5 \\
\hline $48.9-50.9$ & $160.5-167$ & Bedded Tuff: Vitric. & $\begin{array}{l}\text { Ammonia Tanks Tuff } \\
\text { (bedded), } \\
\text { Timber Mountain Group }\end{array}$ & $\operatorname{Tma}(\mathrm{b})$ & 2.0 & 6.5 \\
\hline
\end{tabular}




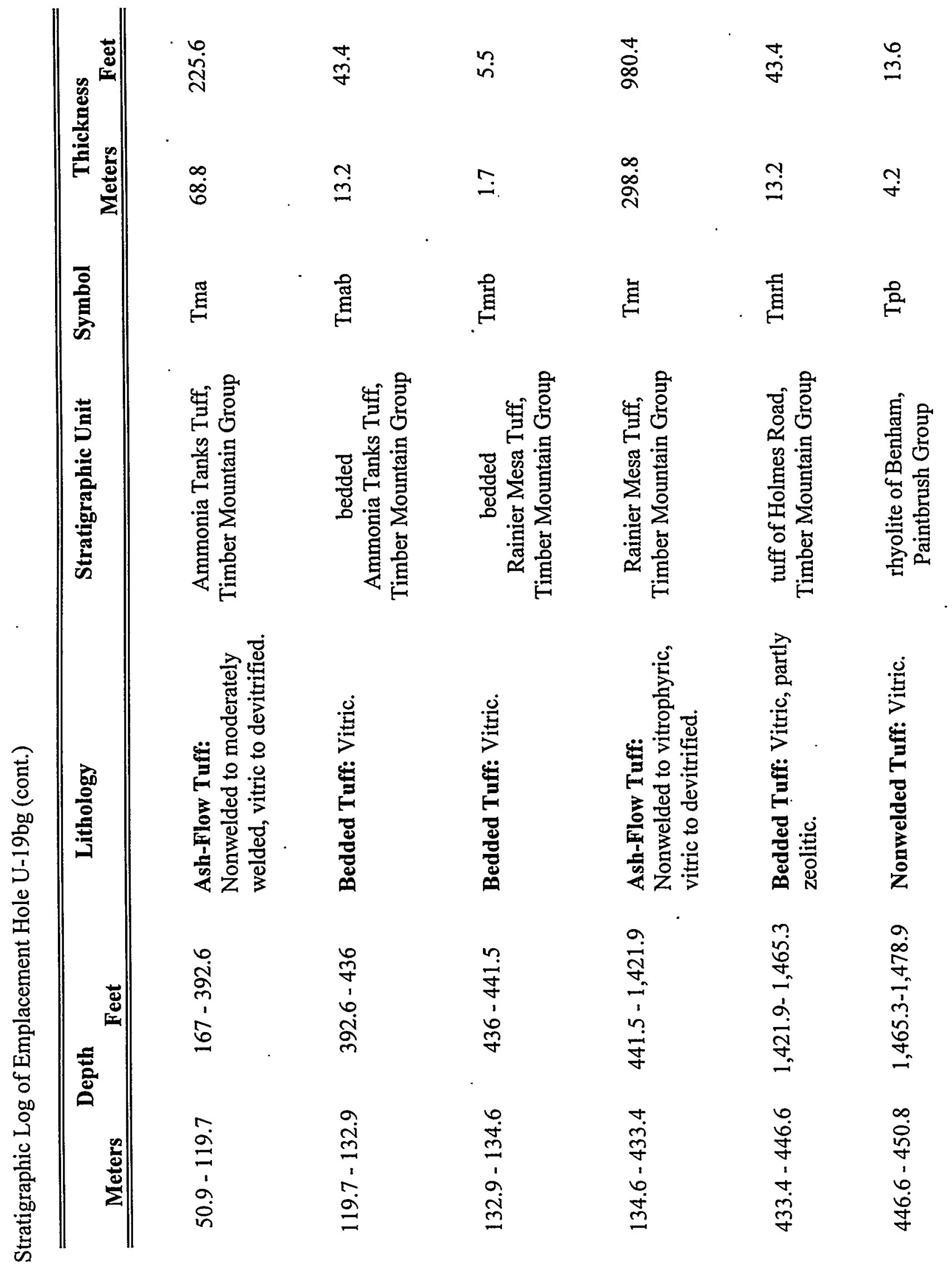




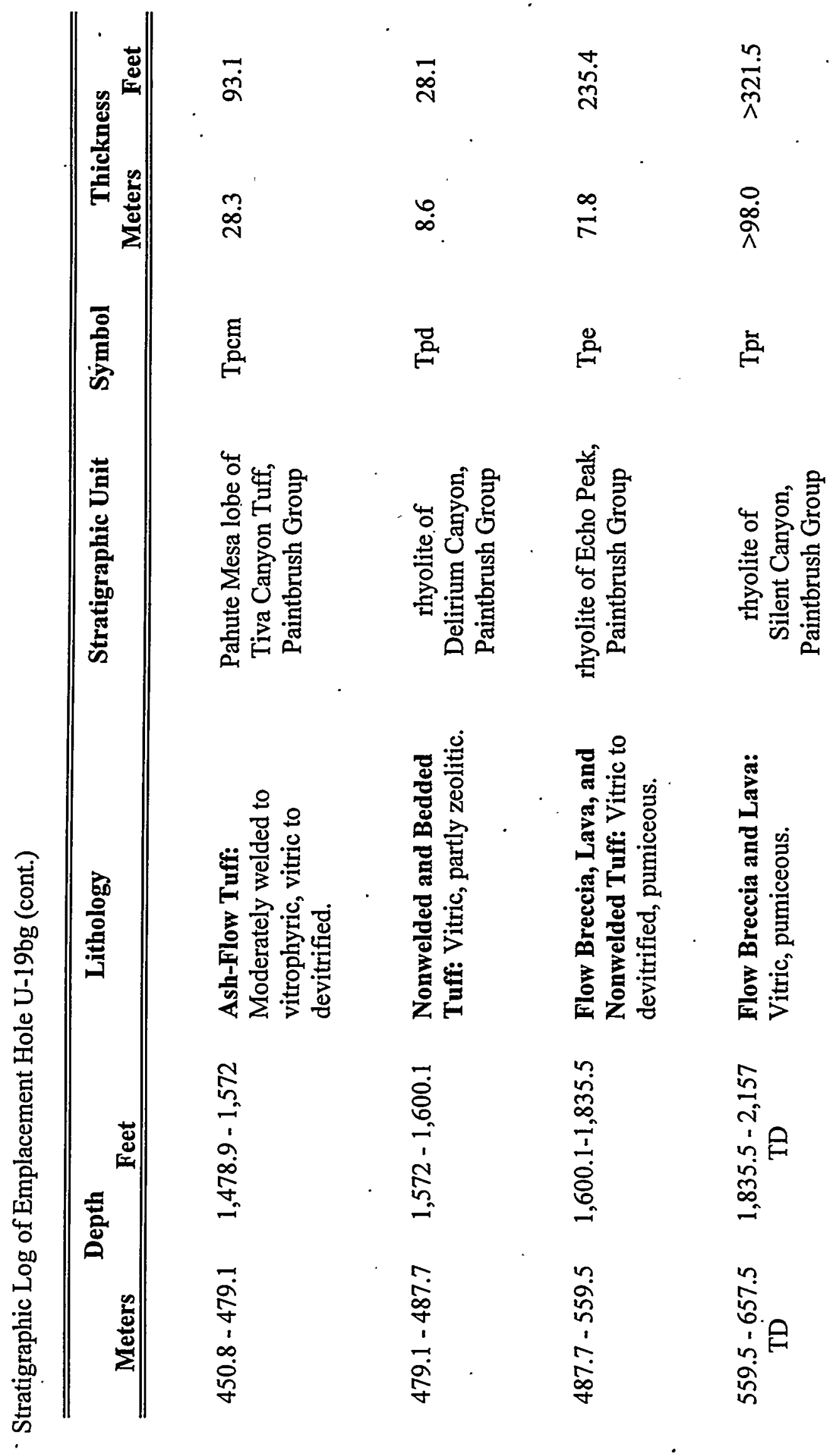




\section{Lithologic Log of Emplacement Hole U-19bg}

Logged by Lance Prothro, Raytheon Services Nevada

August 1991

Unless otherwise noted, the following descriptions refer to washed cuttings samples at 3.05-m (10-ft) intervals. Colors are determined by comparing wet sample color to the Geological Society of America Rock-Color Chart. Stratigraphic contacts and lithologic divisions are tied to geophysical logs whenever possible. Geologic information gathered from the LANL Borehole Inspection System video and LLNL 16-mm film, and notes from R.G. Warren have been incorporated into the descriptions.

\begin{tabular}{ccc}
$\begin{array}{c}\text { Depth } \\
\text { Meters/(feet) }\end{array}$ & Lithologic Description & Stratigraphic Unit \\
\hline
\end{tabular}

$0-9.1 \mathrm{~m}$

$(0-30 \mathrm{ft})$

$9.1-29.0 \mathrm{~m}$ $(30-95.3 \mathrm{ft})$

$29.0-32.9 \mathrm{~m}$

$(95.3-108 \mathrm{ft})$
Ash-Flow tuff: No samples. (Stratigraphy inferred from Noble et al., USGS Geologic Map of the Dead Horse Flat quadrangle, 1967.)

Partially Welded Ash-Flow Tuff: Pale-brown to grayish-red (5R4/2); devitrified with vapor-phase mineralization; common medium-gray pumice; minor felsic phenocrysts of sanidine; rare mafic minerals of clinopyroxene and olivine; minor grayish-brown lithic fragments up to $1 \mathrm{~mm}$ in diameter. Moderate fractures emerging from behind casing to $29.0 \mathrm{~m}$ (95.3 ft).

Bedded Tuff: Light (5YR5/6) to moderate (5YR4/4) brown; poorly indurated; vitric; minor very paleorange pumice; common felsic phenocrysts of feldspar and lesser quartz; minor mafic minerals of magnetite and hornblende with lesser biotite and pyroxene; minor darkreddish-brown and light-bluish-gray lithic fragments up to $5 \mathrm{~mm}$ in diameter. Upper contact strikes approximately $\mathrm{N} 40^{\circ} \mathrm{E}$ and dips $6^{\circ} \mathrm{SE}$.
Thirsty Canyon Group, undifferentiated

Pahute Mesa Tuff

rhyolite of Chukar Canyon 


\begin{tabular}{|c|c|c|}
\hline $\begin{array}{c}\text { Depth } \\
\text { Meters/(feet) }\end{array}$ & Lithologic Description & Stratigraphic Unit \\
\hline $\begin{array}{c}32.9-36.0 \mathrm{~m} \\
(108-118 \mathrm{ft}) \\
\end{array}$ & $\begin{array}{l}\text { Bedded Tuff: Pale-yellowish-brown; } \\
\text { poorly indurated; partly vitric; partly } \\
\text { zeolitic; moderately calcareous; } \\
\text { abundant white pumice; common felsic } \\
\text { phenocrysts of feldspar and lesser } \\
\text { quartz; rare mafic minerals of } \\
\text { magnetite, hornblende and biotite; } \\
\text { common brownish-gray to very-light- } \\
\text { gray lithic fragments up to } 5 \mathrm{~mm} \text { in } \\
\text { diameter. Trace of sphene in } \\
\text { percussion gun sample at } 35.4 \mathrm{~m} \\
\text { (116 ft). }\end{array}$ & $\begin{array}{l}\text { rhyolite of } \\
\text { Chukar Canyon }\end{array}$ \\
\hline $\begin{array}{c}36.0-48.9 \mathrm{~m} \\
(118-160.5 \mathrm{ft})\end{array}$ & $\begin{array}{l}\text { Bedded Tuff: White and pale- } \\
\text { yellowish-brown; vitric; abundant } \\
\text { white pumice; minor felsic phenocrysts } \\
\text { of feldspar; minor mafic minerals of } \\
\text { biotite, hornblende and magnetite; } \\
\text { minor pale-reddish-brown lithic } \\
\text { fragments; trace of sphene. } \\
\text { Description is from percussion gun } \\
\text { samples at } 36.6 \mathrm{~m} \text { (120 ft) and } 47.8 \mathrm{~m} \\
(157 \mathrm{ft}) \text {. }\end{array}$ & $\begin{array}{l}\text { rhyolite of } \\
\text { Beatty Wash }\end{array}$ \\
\hline $\begin{array}{c}48.9-50.9 \mathrm{~m} \\
(160.5-167 \mathrm{ft})\end{array}$ & $\begin{array}{l}\text { Bedded Tuff: Moderate-yellowish- } \\
\text { brown; vitric; minor white pumice; } \\
\text { abundant felsic phenocrysts of } \\
\text { feldspar; minor mafic minerals of } \\
\text { biotite, clinopyroxene and magnetite; } \\
\text { minor moderate-red }(5 \mathrm{R} 4 / 6) \text { and } \\
\text { grayish-red-purple lithic fragments up } \\
\text { to } 10 \mathrm{~mm} \text { in diameter. Upper contact } \\
\text { strikes approximately N55 } 5^{\circ} \mathrm{E} \text { and dips } \\
6^{\circ} \mathrm{SE} \text {. }\end{array}$ & $\begin{array}{c}\text { mafic-rich } \\
\text { Ammonia Tanks Tuff } \\
\text { (bedded) }\end{array}$ \\
\hline
\end{tabular}


Lithologic Log of Emplacement Hole U-19bg (cont.)

\begin{tabular}{|c|c|c|}
\hline $\begin{array}{c}\text { Depth } \\
\text { Meters/(feet) }\end{array}$ & Lithologic Description & Stratigraphic Unit \\
\hline $\begin{array}{c}50.9-60.2 \mathrm{~m} \\
(167-197.6 \mathrm{ft})\end{array}$ & $\begin{array}{l}\text { Nonwelded Ash-Flow Tuff: } \\
\text { Moderate-reddish-brown; vitric; minor } \\
\text { white pumice; minor felsic phenocrysts } \\
\text { of feldspar and much less quartz; } \\
\text { common mafic minerals of biotite with } \\
\text { lesser clinopyroxene and magnetite; } \\
\text { minor light-brownish-gray and } \\
\text { moderate-red ( } 5 \mathrm{R} 4 / 6) \text { lithic fragments } \\
\text { up to } 3 \mathrm{~mm} \text { in diameter. Percussion } \\
\text { gun sample at } 51.8 \mathrm{~m}(170 \mathrm{ft}) \\
\text { contained very abundant white pumice } \\
\text { and a trace of sphene. }\end{array}$ & $\begin{array}{c}\text { mafic-rich } \\
\text { Ammonia Tanks Tuff }\end{array}$ \\
\hline $\begin{array}{c}60.2-69.8 \mathrm{~m} \\
(197.6-229 \mathrm{ft})\end{array}$ & 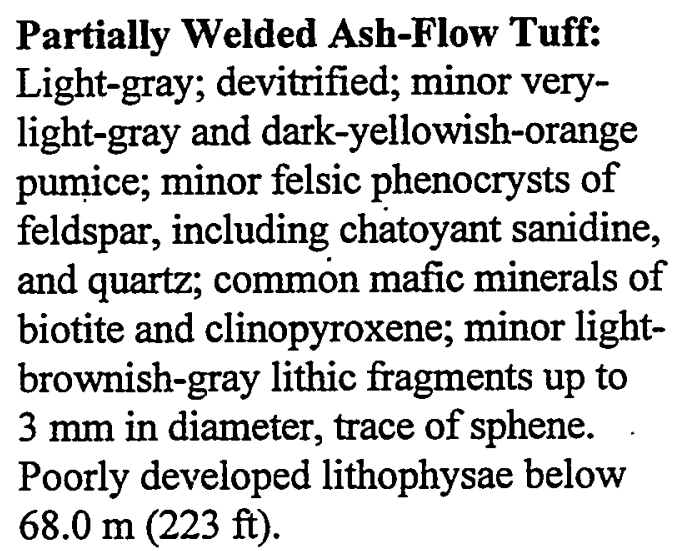 & $\begin{array}{c}\text { mafic-rich } \\
\text { Ammonia Tanks Tuff }\end{array}$ \\
\hline
\end{tabular}


Lithologic Log of Emplacement Hole U-19bg (cont.)

\begin{tabular}{|c|c|c|}
\hline $\begin{array}{c}\text { Depth } \\
\text { Meters/(feet) }\end{array}$ & Lithologic Description & Stratigraphic Unit \\
\hline $\begin{array}{l}69.8-102.4 \mathrm{~m} \\
(229-336 \mathrm{ft})\end{array}$ & $\begin{array}{l}\text { Partially Welded Ash-Flow Tuff: } \\
\text { Light-gray; devitrified; minor very- } \\
\text { light-gray and dark-yellowish-orange } \\
\text { pumice; common felsic phenocrysts of } \\
\text { feldspar, including chatoyant sanidine, } \\
\text { and quartz; rare to minor mafic } \\
\text { minerals of biotite, clinopyroxene, and } \\
\text { magnetite; minor light-brownish-gray } \\
\text { lithic fragments up to } 3 \mathrm{~mm} \text { in } \\
\text { diameter; trace of sphene. Moderate } \\
\text { fractures below } 90.0 \mathrm{~m} \text { ( } 295.3 \mathrm{ft} \text { ). } \\
\text { Poorly developed lithophysae from } \\
\text { approximately } 69.8-85.3 \mathrm{~m} \text { ( } 229 \text { - } \\
280 \mathrm{ft})\end{array}$ & $\begin{array}{c}\text { mafic-poor } \\
\text { Ammonia Tanks Tuff }\end{array}$ \\
\hline $\begin{array}{l}102.4-119.7 \mathrm{~m} \\
(336-392.6 \mathrm{ft})\end{array}$ & $\begin{array}{l}\text { Moderately Welded Ash-Flow Tuff: } \\
\text { Medium-light-gray to pale-brown; } \\
\text { devitrified with vapor-phase } \\
\text { mineralization; approaching densely } \\
\text { welded from } 113.7 \text { - } 115.8 \mathrm{~m}(373- \\
380 \mathrm{ft}) \text {; minor yellowish-gray ( } 5 \mathrm{Y} 7 / 2) \\
\text { pumice; common felsic phenocrysts of } \\
\text { feldspar, including chatoyant sanidine, } \\
\text { and quartz; rare mafic minerals of } \\
\text { biotite and magnetite; rare grayish- } \\
\text { brown lithic fragments up to } 3 \mathrm{~mm} \text { in } \\
\text { diameter. Moderate fractures from } \\
102.4 \text { - } 116.9 \mathrm{~m}(336 \text { - } 383.6 \mathrm{ft}) .\end{array}$ & $\begin{array}{c}\text { mafic-poor } \\
\text { Ammonia Tanks Tuff }\end{array}$ \\
\hline
\end{tabular}


Lithologic Log of Emplacement Hole U-19bg (cont.)

\begin{tabular}{|c|c|c|}
\hline $\begin{array}{c}\text { Depth } \\
\text { Meters/(feet) }\end{array}$ & Lithologic Description & Stratigraphic Unit \\
\hline $\begin{array}{l}119.7-132.9 \mathrm{~m} \\
(392.6-436 \mathrm{ft})\end{array}$ & $\begin{array}{l}\text { Bedded Tuff: White, grayish-orange, } \\
\text { and moderate-brown (5YR4/4); vitric; } \\
\text { minor white to light-brown ( } 5 \text { YR } 5 / 6) \\
\text { pumice becoming abundant and mostly } \\
\text { white near base of interval; common } \\
\text { felsic phenocrysts of feldspar and } \\
\text { lesser quartz; minor mafic minerals of } \\
\text { biotite and magnetite; rare pale-red- } \\
\text { purple and light-bluish-gray lithic } \\
\text { fragments up to } 3 \mathrm{~mm} \text { in diameter; } \\
\text { trace of sphene. Description is from } \\
\text { percussion gun samples at } 130.1 \mathrm{~m} \\
(427 \mathrm{ft}), 131.7 \mathrm{~m}(432 \mathrm{ft}), \text { and } 132.3 \mathrm{~m} \\
\text { ( } 434 \mathrm{ft}) \text {. Hornblende was observed in } \\
\text { the cuttings samples from this interval. }\end{array}$ & $\begin{array}{c}\text { bedded } \\
\text { Ammonia Tanks tuff }\end{array}$ \\
\hline $\begin{array}{l}132.9-134.6 \mathrm{~m} \\
(436-441.5 \mathrm{ft})\end{array}$ & $\begin{array}{l}\text { Bedded Tuff: Not represented in } \\
\text { cuttings. Stratigraphic contacts from } \\
\text { geophysical logs. Percussion gun } \\
\text { sample at } 133.5 \mathrm{~m} \text { ( } 438 \mathrm{ft}) \text { is } \\
\text { moderately to well sorted, medium- } \\
\text { grained ash: Pale-reddish-brown; } \\
\text { partly vitric, partly zeolitic; minor } \\
\text { felsic phenocrysts; minor biotite; minor } \\
\text { lithic fragments less than } 1 \mathrm{~mm} \text { in } \\
\text { diameter. }\end{array}$ & $\begin{array}{c}\text { bedded } \\
\text { Rainier Mesa Tuff }\end{array}$ \\
\hline $\begin{array}{c}134.6-143.2 \mathrm{~m} \\
(441.5-469.8 \mathrm{ft})\end{array}$ & $\begin{array}{l}\text { Nonwelded Tuff: Moderate-brown } \\
\text { ( } 5 \text { YR 4/4); poorly indurated; vitric; } \\
\text { common white to grayish-orange } \\
\text { pumice; common felsic phenocrysts of } \\
\text { feldspar and quartz; common mafic } \\
\text { minerals of biotite, clinopyroxene and } \\
\text { lesser orthopyroxene, rare pale-reddish- } \\
\text { brown lithic fragments up to } 2 \mathrm{~mm} \text { in } \\
\text { diameter. }\end{array}$ & $\begin{array}{c}\text { mafic-rich } \\
\text { Rainier Mesa Tuff }\end{array}$ \\
\hline
\end{tabular}




\begin{tabular}{|c|c|c|}
\hline $\begin{array}{c}\text { Depth } \\
\text { Meters/(feet) }\end{array}$ & Lithologic Description & Stratigraphic Unit \\
\hline $\begin{array}{l}143.2-154.2 \mathrm{~m} \\
(469.8-506 \mathrm{ft})\end{array}$ & $\begin{array}{l}\text { Partially Welded Ash-Flow Tuff: } \\
\text { Grayish-red (10R4/2); devitrified with } \\
\text { vapor-phase mineralization; minor } \\
\text { medium-light-gray pumice; common } \\
\text { felsic phenocrysts of feldspar and } \\
\text { quartz; abundant mafic minerals of } \\
\text { biotite, clinopyroxene and } \\
\text { orthopyroxene; rare lithic fragments up } \\
\text { to } 1 \text { mm in diameter. Poorly developed } \\
\text { fractures throughout interval. }\end{array}$ & $\begin{array}{c}\text { mafic-rich } \\
\text { Rainier Mesa Tuff }\end{array}$ \\
\hline $\begin{array}{c}154.2-159.1 \mathrm{~m} \\
(506-522 \mathrm{ft})\end{array}$ & $\begin{array}{l}\text { Moderately Welded Ash-Flow Tuff: } \\
\text { Pale-red (5R6/2); devitrified with } \\
\text { vapor-phase mineralization; minor } \\
\text { light-gray pumice; common felsic } \\
\text { phenocrysts of feldspar and quartz; } \\
\text { abundant mafic minerals of biotite, } \\
\text { clinopyroxene and orthopyroxene; rare } \\
\text { lithic fragments up to } 1 \mathrm{~mm} \text { in } \\
\text { diameter. Poorly developed fractures } \\
\text { to } 156.9 \mathrm{~m} \text { ( } 514.6 \mathrm{ft} \text { ) becoming } \\
\text { moderately developed throughout } \\
\text { remaining interval. }\end{array}$ & $\begin{array}{c}\text { mafic-rich } \\
\text { Rainier Mesa Tuff }\end{array}$ \\
\hline $\begin{array}{c}159.1-164.6 \mathrm{~m} \\
(522-540 \mathrm{ft})\end{array}$ & $\begin{array}{l}\text { Densely Welded Ash-Flow Tuff: } \\
\text { Moderate-brown; mostly devitrified; } \\
\text { minor white to pale-pink pumice; } \\
\text { common felsic phenocrysts of feldspar } \\
\text { and quartz; abundant mafic minerals of } \\
\text { biotite and clinopyroxene; rare lithic } \\
\text { fragments; black to medium-dark-gray } \\
\text { glass sphericles. Moderate fractures } \\
\text { throughout. }\end{array}$ & $\begin{array}{c}\text { mafic-rich } \\
\text { Rainier Mesa Tuff }\end{array}$ \\
\hline
\end{tabular}


Lithologic Log of Emplacement Hole U-19bg (cont.)

\begin{tabular}{|c|c|c|}
\hline $\begin{array}{c}\text { Depth } \\
\text { Meters/(feet) }\end{array}$ & Lithologic Description & Stratigraphic Unit \\
\hline $\begin{array}{c}164.6-167.3 \mathrm{~m} \\
(540-549 \mathrm{ft})\end{array}$ & $\begin{array}{l}\text { Moderately Welded Ash-Flow Tuff: } \\
\text { Pale-brown; devitrified; minor white } \\
\text { pumice; common felsic phenocrysts of } \\
\text { feldspar and quartz; abundant mafic } \\
\text { minerals of biotite and clinopyroxene; } \\
\text { rare lithic fragments. Moderate } \\
\text { fractures throughout. }\end{array}$ & $\begin{array}{c}\text { mafic-rich } \\
\text { Rainier Mesa Tuff }\end{array}$ \\
\hline $\begin{array}{c}167.3-182.9 \mathrm{~m} \\
(549-600 \mathrm{ft})\end{array}$ & $\begin{array}{l}\text { Moderately Welded Ash-Flow Tuff: } \\
\text { Pale-brown; devitrified; minor white } \\
\text { pumice; minor felsic phenocrysts of } \\
\text { feldspar and quartz; minor biotite; rare } \\
\text { lithic fragments. Poorly developed } \\
\text { fractures throughout. }\end{array}$ & $\begin{array}{c}\text { mafic-poor } \\
\text { Rainier Mesa Tuff }\end{array}$ \\
\hline $\begin{array}{c}182.9-194.5 \mathrm{~m} \\
(600-638 \mathrm{ft})\end{array}$ & $\begin{array}{l}\text { Densely Welded Ash-Flow Tuff: } \\
\text { Grayish-red (5R } 4 / 2) \text {; devitrified; minor } \\
\text { white to medium-gray pumice; minor } \\
\text { felsic phenocrysts of feldspar and } \\
\text { quartz; minor mafic minerals of biotite; } \\
\text { rare lithic fragments. Poorly developed } \\
\text { fractures to } 190.0 \mathrm{~m}(623.2 \mathrm{ft}) \text {. }\end{array}$ & $\begin{array}{c}\text { mafic-poor } \\
\text { Rainier Mesa Tuff }\end{array}$ \\
\hline
\end{tabular}


Lithologic Log of Emplacement Hole U-19bg (cont.)

\begin{tabular}{|c|c|c|}
\hline $\begin{array}{c}\text { Depth } \\
\text { Meters/(feet) }\end{array}$ & Lithologic Description & Stratigraphic Unit \\
\hline $\begin{array}{l}194.5-373.1 \mathrm{~m} \\
(638-1,224 \mathrm{ft})\end{array}$ & $\begin{array}{l}\text { Moderately Welded Ash-Flow Tuff: } \\
\text { Grayish-red (10R4/2); devitrified; } \\
\text { common white and light-bluish-gray } \\
\text { pumice; minor to common felsic } \\
\text { phenocrysts of feldspar and quartz; rare } \\
\text { to minor biotite; rare lithic fragments. } \\
\text { Moderate fractures from } 229.1 \text { - } \\
245.2 \mathrm{~m} \text { ( } 751.6 \text { - } 804.6 \mathrm{ft}) \text {. Poorly } \\
\text { developed fractures from } 194.7- \\
201.2 \mathrm{~m} \text { ( } 638.9-660.2 \mathrm{ft}), 245.2-\text {. } \\
255.8 \mathrm{~m} \text { ( } 804.6-839.2 \mathrm{ft}) \text {, and below } \\
366.1 \mathrm{~m} \text { (1,201 ft). Moderately } \\
\text { developed lithophysae from } 201.2 \text { - } \\
221.7 \mathrm{~m} \text { ( } 660.2-727.2 \mathrm{ft}) \text { and below } \\
303.9 \mathrm{~m} \text { ( } 997 \mathrm{ft}) \text {. Poorly developed } \\
\text { lithophysae from } 280.2-297.0 \mathrm{~m}(919- \\
974 \mathrm{ft}) \text {. }\end{array}$ & $\begin{array}{l}\text { mafic-poor } \\
\text { Rainier Mesa Tuff }\end{array}$ \\
\hline $\begin{array}{c}373.1-388.6 \mathrm{~m} \\
(1,224-1,275.1 \mathrm{ft})\end{array}$ & $\begin{array}{l}\text { Densely Welded Ash-Flow Tuff: } \\
\text { Grayish-red (5R4/2); devitrified; minor } \\
\text { medium-light-gray to medium-bluish- } \\
\text { gray pumice; minor felsic phenocrysts } \\
\text { of feldspar and quartz; rare biotite; } \\
\text { trace of lithic fragments. Poorly } \\
\text { developed fractures to } 387.4 \mathrm{~m} \\
\text { (1,271 ft). Moderately developed } \\
\text { lithophysae throughout interval. }\end{array}$ & $\begin{array}{c}\text { mafic-poor } \\
\text { Rainier Mesa Tuff }\end{array}$ \\
\hline $\begin{array}{c}388.6-395.0 \mathrm{~m} \\
(1,275.1-1,296 \mathrm{ft})\end{array}$ & $\begin{array}{l}\text { Vitrophyre: Grayish-black; vitric; } \\
\text { minor felsic phenocrysts of feldspar } \\
\text { and quartz; minor biotite; trace of lithic } \\
\text { fragments. Directional break-out } \\
\text { oriented } \mathrm{N} 80^{\circ} \mathrm{W} \text { throughout interval. }\end{array}$ & $\begin{array}{c}\text { mafic-poor } \\
\text { Rainier Mesa Tuff }\end{array}$ \\
\hline
\end{tabular}


Lithologic Log of Emplacement Hole U-19bg (cont.)

\begin{tabular}{|c|c|c|}
\hline $\begin{array}{c}\text { Depth } \\
\text { Meters/(feet) }\end{array}$ & Lithologic Description & Stratigraphic Unit \\
\hline $\begin{array}{c}395.0-405.3 \mathrm{~m} \\
(1,296-1,329.6 \mathrm{ft})\end{array}$ & $\begin{array}{l}\text { Moderately Welded Ash-Flow Tuff: } \\
\text { Moderate-reddish-brown; vitric; } \\
\text { becoming moderately welded at base; } \\
\text { minor dark-gray to medium-light-gray } \\
\text { pumice; minor felsic phenocrysts of } \\
\text { feldspar and quartz; minor biotite; rare } \\
\text { lithic fragments up to } 2 \mathrm{~mm} \text { in } \\
\text { diameter; common black to medium- } \\
\text { gray glass sphericles. Directional } \\
\text { break-out oriented } \mathrm{N} 80^{\circ} \mathrm{W} \text { from } 395.0 \text { - } \\
402.7 \mathrm{~m}(1,296-1,321.2 \mathrm{ft}) \text {. }\end{array}$ & $\begin{array}{c}\text { mafic-poor } \\
\text { Rainier Mesa Tuff }\end{array}$ \\
\hline $\begin{array}{c}405.3-408.1 \mathrm{~m} \\
(1,329.6-1,339 \mathrm{ft})\end{array}$ & $\begin{array}{l}\text { Partially Welded Ash-Flow Tuff: } \\
\text { Pale-reddish-brown; vitric; minor } \\
\text { medium-light-gray pumice; minor } \\
\text { felsic phenocrysts of feldspar and } \\
\text { quartz; rare biotite; minor pale-yellow } \\
\text { to moderate-yellow, vesiculated glass } \\
\text { shards. }\end{array}$ & $\begin{array}{c}\text { mafic-poor } \\
\text { Rainier Mesa Tuff }\end{array}$ \\
\hline $\begin{array}{c}408.1-433.4 \mathrm{~m} \\
(1,339-1,421.9 \mathrm{ft})\end{array}$ & $\begin{array}{l}\text { Nonwelded Ash-Flow Tuff: Samples } \\
\text { are a concentrate of predominately } \\
\text { felsic minerals and pumice; moderate- } \\
\text { reddish-orange (color obtained from } \\
\text { thin matrix coatings on pumice and } \\
\text { glass shards); vitric; white pumice; } \\
\text { felsic minerals of feldspar and quartz; } \\
\text { mafic minerals of biotite and lesser } \\
\text { orthopyroxene; moderate to dark- } \\
\text { reddish-brown lithic fragments up to } \\
2 \text { mm in diameter; grayish-yellow to } \\
\text { very-pale-orange vesiculated glass } \\
\text { shards which markedly decrease in } \\
\text { abundance below approximately } \\
414.5 \mathrm{~m} \text { (1,360 ft). }\end{array}$ & $\begin{array}{c}\text { mafic-poor } \\
\text { Rainier Mesa tuff }\end{array}$ \\
\hline
\end{tabular}


Lithologic Log of Emplacement Hole U-19bg (cont.)

\begin{tabular}{|c|c|c|}
\hline $\begin{array}{c}\text { Depth } \\
\text { Meters/(feet) }\end{array}$ & Lithologic Description & Stratigraphic Unit \\
\hline $\begin{array}{c}433.4-438.2 \mathrm{~m} \\
(1,421.9-1,437.5 \mathrm{ft})\end{array}$ & $\begin{array}{l}\text { Bedded Tuff: Grayish-orange-pink; } \\
\text { vitric; abundant white to moderate- } \\
\text { orange-pink pumice at } 434.0 \mathrm{~m} \\
(1,424 \mathrm{ft}) \text {, absent at } 436.2 \mathrm{~m}(1,431 \mathrm{ft}) \text {; } \\
\text { minor felsic phenocrysts of feldspar } \\
\text { and quartz; rare biotite; minor } \\
\text { moderate-red lithic fragments up to } \\
4 \mathrm{~mm} \text { in diameter. Description is from } \\
\text { percussion gun samples at } 434.0 \mathrm{~m} \\
(1,424 \mathrm{ft}) \text { and } 436.2 \mathrm{~m}(1,431 \mathrm{ft}) \text {. }\end{array}$ & tuff of Holmes Road \\
\hline $\begin{array}{c}438.2-446.6 \mathrm{~m} \\
(1,437.5-1,465.3 \mathrm{ft})\end{array}$ & $\begin{array}{l}\text { Bedded Tuff: Moderate-reddish- } \\
\text { brown; poorly indurated; partly vitric; } \\
\text { partly zeolitic; slightly calcareous; } \\
\text { minor white, mostly vitric pumice; } \\
\text { minor felsic phenocrysts of feldspar } \\
\text { and quartz; rare to minor mafic } \\
\text { minerals of biotite, hornblende, } \\
\text { clinopyroxene and magnetite; minor } \\
\text { pale to dark-reddish-brown and } \\
\text { medium-light-gray lithic fragments up } \\
\text { to } 2 \text { mm in diameter; minor very-pale- } \\
\text { orange hydroclastic glass shards. } \\
\text { Bedding is approximately horizontal } \\
\text { throughout interval. }\end{array}$ & tuff of Holmes Road \\
\hline
\end{tabular}


Lithologic Log of Emplacement Hole U-19bg (cont.)

\begin{tabular}{ccc}
\hline $\begin{array}{c}\text { Depth } \\
\text { Meters/(feet) }\end{array}$ & Lithologic Description & Stratigraphic Unit \\
\hline
\end{tabular}

$446.6-450.8 \mathrm{~m}$ $(1,465.3-1,478.9 \mathrm{ft})$

$450.8-452.4 \mathrm{~m}$ $(1,478.9-1,484.4 \mathrm{ft})$

$452.4-455.5 \mathrm{~m}$ $(1,484.4-1,494.5 \mathrm{ft})$

$455.5-460.9 \mathrm{~m}$ $(1,494.5-1,512 \mathrm{ft})$
Nonwelded Tuff: Very-pale-orange; vitric; common felsic phenocrysts of feldspar (grain mount by Warren indicated 12 percent total felsic phenocrysts of which 60 percent were potassium feldspar and 40 percent plagioclase; quartz absent); minor biotite and a trace of hornblende; minor moderate-brown (5YR3/4) lithic fragments up to $4 \mathrm{~mm}$ in diameter; conspicuous sphene. Description is from percussion gun sample at $449.3 \mathrm{~m}$ $(1,474 \mathrm{ft})$.

Moderately Welded Ash-Flow Tuff: Moderate-brown (5YR3/4); vitric; rare very-light-gray pumice; rare felsic phenocrysts of feldspar; rare mafic minerals of biotite, clinopyroxene and magnetite.

Vitrophyre: Dusky-yellowish-brown; vitric; minor felsic phenocrysts of feldspar; rare biotite; trace of mediumgray lithic fragments; common darkreddish-brown glass shards.

Densely Welded Ash-Flow Tuff: Dark-yellowish-brown; vitric; rare felsic phenocrysts of feldspar; rare biotite; trace of chalcedony. rhyolite of Benham

Pahute Mesa lobe of Tiva Canyon Tuff

Pahute Mesa lobe of Tiva Canyon Tuff

Pahute Mesa lobe of Tiva Canyon Tuff 


\begin{tabular}{|c|c|c|}
\hline $\begin{array}{c}\text { Depth } \\
\text { Meters/(feet) }\end{array}$ & Lithologic Description & Stratigraphic Unit \\
\hline $\begin{array}{c}460.9-479.1 \mathrm{~m} \\
(1,512-1,572 \mathrm{ft})\end{array}$ & $\begin{array}{l}\text { Moderately Welded Ash-Flow Tuff: } \\
\text { Dark-yellowish-brown to pale-brown; } \\
\text { devitrified with vapor-phase } \\
\text { mineralization below } 472.4 \mathrm{~m} \\
\text { (1,550 ft); rare yellowish-gray ( } 5 Y 8 / 1) \\
\text { pumice; rare felsic phenocrysts of } \\
\text { feldspar; rare mafic minerals of biotite, } \\
\text { clinopyroxene and lesser hornblende; } \\
\text { trace of sphene below } 472.4 \mathrm{~m} \\
(1,550 \mathrm{ft}) .\end{array}$ & $\begin{array}{c}\text { Pahute Mesa lobe of } \\
\text { Tiva Canyon Tuff }\end{array}$ \\
\hline $\begin{array}{c}479.1-484.0 \mathrm{~m} \\
(1,572-1,587.9 \mathrm{ft})\end{array}$ & $\begin{array}{l}\text { Nonwelded Tuff: ,Sample is a } \\
\text { concentrate of mainly felsic crystals, } \\
\text { pumice, and lithic fragments; white to } \\
\text { yellowish-gray (color obtained from } \\
\text { matrix coatings on lithic fragments); } \\
\text { vitric; white pumice; felsic crystals of } \\
\text { feldspar and dipyramidal quartz; mafic } \\
\text { minerals of biotite, hornblende, } \\
\text { magnetite and a trace of clinopyroxene; } \\
\text { moderate-reddish-brown to grayish- } \\
\text { brown lithic fragments up to } 4 \text { mm in } \\
\text { diameter. Percussion gun sample at } \\
483.1 \text { m ( } 1,585 \mathrm{ft} \text { ): Light-gray-orange- } \\
\text { pink; vitric; minor felsic phenocrysts of } \\
\text { feldspar; rare mafic minerals of } \\
\text { hornblende and lesser biotite; trace of } \\
\text { sphene. }\end{array}$ & $\begin{array}{c}\text { rhyolite of } \\
\text { Delirium Canyon }\end{array}$ \\
\hline
\end{tabular}


Lithologic Log of Emplacement Hole U-19bg (cont.)

Depth

Lithologic Description

Stratigraphic Unit

Meters/(feet)

$484.0-487.7 \mathrm{~m} \quad$ Bedded Tuff: Light-brown (5YR5/6);

$(1,587.9-1,600.1 \mathrm{ft})$

poorly indurated; partly vitric; partly

zeolitic; rare felsic phenocrysts of

feldspar; rare biotite, hornblende and

magnetite; trace of lithic fragments;

clear vesiculated glass shards showing

varying degrees of devitrification

and/or zeolitization. Washout

preferentially oriented $\mathrm{N} 10^{\circ} \mathrm{E}$ from

485.1 - $487.7 \mathrm{~m}(1,591.4-1,600.1 \mathrm{ft})$.

$487.7-500.0 \mathrm{~m}$

$(1,600.1-1,640.3 \mathrm{ft})$

Flow Breccia: Light-gray; poorly to

rhyolite of

moderately indurated; vitric; minor

Echo Peak

felsic phenocrysts of feldspar; rare

mafic minerals of biotite and magnetite with a trace of clinopyroxene; trace of sphene. Upper contact is irregular and strikes approximately $\mathrm{N} 60^{\circ} \mathrm{W}$ and dips $31^{\circ} \mathrm{SW}$.

$500.0-526.1 \mathrm{~m}$

Lava: Medium-gray to brownish-gray;

rhyolite of $(1,640.3-1,726 \mathrm{ft})$ well indurated; devitrified; minor felsic Echo Peak phenocrysts of feldspar; rare mafic minerals of biotite and magnetite with a trace of clinopyroxene; trace of sphene. 
Lithologic Log of Emplacement Hole U-19bg (cont.)

Depth

Meters/(feet)
Lithologic Description

Flow Breccia: Very-light-gray to

grayish-black; poorly to moderately

indurated; vitric; becomes pumiceous

below $542.5 \mathrm{~m}$ (1,779.7 ft); minor

felsic phenocrysts of feldspar; rare

mafic minerals of biotite and magnetite

with a trace of clinopyroxene;

conspicuous sphene; common black

glass sphericles. Note: Sample 545.6 -

$548.6 \mathrm{~m}(1,790-1,800 \mathrm{ft})$ is

contaminated with rhyolite of Delirium

Canyon (Tpd) due to sloughing from

approximately $484.0-487.7 \mathrm{~m}$

$(1,587.9-1,600.1 \mathrm{ft})$.

Stratigraphic Unit

rhyolite of

Echo Peak

$552.9-559.5 \mathrm{~m}$

$(1,814-1,835.5 \mathrm{ft})$
Nonwelded Tuff: White; poorly

indurated; vitric; minor felsic phenocrysts of feldspar; rare mafic minerals of biotite and magnetite with a trace of free clinopyroxene; minor grayish-red-purple and moderatebrown (5YR3/4) lithic fragments up to $4 \mathrm{~mm}$ in diameter; conspicuous sphene.
$559.5-581.2 \mathrm{~m}$ $(1,835.5-1,906.8 \mathrm{ft})$
Flow Breccia: Light-gray to darkgray; poorly to moderately indurated; vitric; minor felsic phenocrysts of feldspar; common to abundant mafic minerals of biotite and magnetite. rhyolite of

Echo Peak rhyolite of Silent Canyon 
Lithologic Log of Emplacement Hole U-19bg (cont.)

\begin{tabular}{|c|c|c|}
\hline $\begin{array}{c}\text { Depth } \\
\text { Meters/(feet) }\end{array}$ & Lithologic Description & Stratigraphic Unit \\
\hline $\begin{array}{c}581.2-600.8 \mathrm{~m} \\
(1,906.8-1,971 \mathrm{ft})\end{array}$ & $\begin{array}{l}\text { Lava: Light-gray to dark-gray; poorly } \\
\text { to moderately indurated; vitric; minor } \\
\text { felsic phenocrysts of feldspar; common } \\
\text { to abundant mafic minerals of biotite } \\
\text { and magnetite. Poorly developed } \\
\text { fractures throughout. Flow foliation } \\
\text { from } 591.3 \text { - } 597.9 \mathrm{~m} \text { (1,940 - } \\
1,961.7 \mathrm{ft}) \text {. }\end{array}$ & $\begin{array}{l}\text { rhyolite of } \\
\text { Silent Canyon }\end{array}$ \\
\hline $\begin{array}{c}600.8-657.5 \mathrm{~m} \\
(1,971-2,157 \mathrm{ft}) \\
\mathrm{TD}\end{array}$ & $\begin{array}{l}\text { Flow Breccia: Light-gray to dark- } \\
\text { gray; poorly to moderately indurated; } \\
\text { vitric; pumiceous in part; minor felsic } \\
\text { phenocrysts of feldspar; common to } \\
\text { abundant mafic minerals of biotite and } \\
\text { magnetite. Directional breakout } \\
\text { oriented approximately } \mathrm{N} 80^{\circ} \mathrm{W} \text { from } \\
600.8 \text { - } 640.1 \mathrm{~m}(1,971-2,100 \mathrm{ft}) \text {. } \\
\text { Note: Sample } 652.3-655.3 \mathrm{~m}(2,140 \text { - } \\
2,150 \mathrm{ft}) \text { is contaminated with rhyolite } \\
\text { of Echo Peak (Tpe) due to sloughing } \\
\text { from approximately } 522.9-559.5 \mathrm{~m} \\
(1,814-1,835.5 \mathrm{ft}) \text {. }\end{array}$ & $\begin{array}{l}\text { rhyolite of } \\
\text { Silent Canyon }\end{array}$ \\
\hline
\end{tabular}




\section{Emplacement Hole U-19bg Supplemental Data}

1. Downhole photography available: LANL Borehole Inspection System video (Prothro, 1991b); LLNL 16-mm film (Prothro, 1991c); and Sandia color video (Prothro, 1991a).

2. Atlas Wireline Service wireline rotary sidewall cores available for the interval 492.6 $637.6 \mathrm{~m}(1,616-2,092 \mathrm{ft})$ (Prothro, 1991e).

3. Percussion gun sidewall samples available for the interval $35.4-611.1 \mathrm{~m}(116-2,005 \mathrm{ft})$ (Prothro, 1991d).

4. Physical property data (from wireline rotary sidewall samples) (Prothro, 1991h):

\begin{tabular}{lcccc}
\multicolumn{2}{c}{ Depth } & Bulk Density \\
Meters & Feet & $\begin{array}{c}\text { Grain Density } \\
(\mathrm{g} / \mathrm{cc})\end{array}$ & $\begin{array}{c}\text { Moisture Content } \\
(\%)\end{array}$ \\
\hline & & & & \\
492.6 & 1,616 & 142.8 & 2.39 & 1.40 \\
524.3 & 1,720 & 131.8 & 2.39 & 1.72 \\
527.3 & 1,730 & 124.5 & 2.40 & 3.66 \\
543.8 & 1,784 & 131.8 & 2.38 & 7.19 \\
543.8 & $1,794$. & 108.9 & 2.38 & 7.46 \\
557.8 & 1,830 & 91.0 & 2.33 & 17.79 \\
568.4 & 1,865 & 127.5 & 2.41 & 3.90 \\
568.4 & 1,865 & 120.4 & 2.41 & 5.52 \\
576.1 & 1,890 & 119.9 & 2.38 & 5.24 \\
583.4 & 1,914 & 106.7 & 2.41 & 8.72 \\
590.1 & 1,936 & 147.5 & 2.40 & 0.31 \\
590.1 & 1,936 & 148.1 & 2.41 & 0.35 \\
614.5 & 2,016 & 141.0 & 2.40 & 1.35 \\
614.5 & 2,016 & 138.9 & 2.41 & 1.28
\end{tabular}

5. In accordance with Protocols of the Treaty between the U.S. and the former Soviet Union on the Limitation of Underground Nuclear Weapons Tests, geotechnical activities related to verification of the JUNCTION test were conducted by Soviet Designated Personnel at this site (Drellack, 1991).

6. U-19bg Satellite Hole \#1 is located $11.0 \mathrm{~m}(36.1 \mathrm{ft})$ due south of Emplacement Hole U-19bg (Prothro, 1991f).

7. A bottom-hole measurement at U-19bg on 01/30/1992 indicated a total depth of $641.9 \mathrm{~m}$ $(2,106 \mathrm{ft})$, and no fluid present.

8. For additional information on Emplacement Hole U-19bg see Prothro (1991g). 


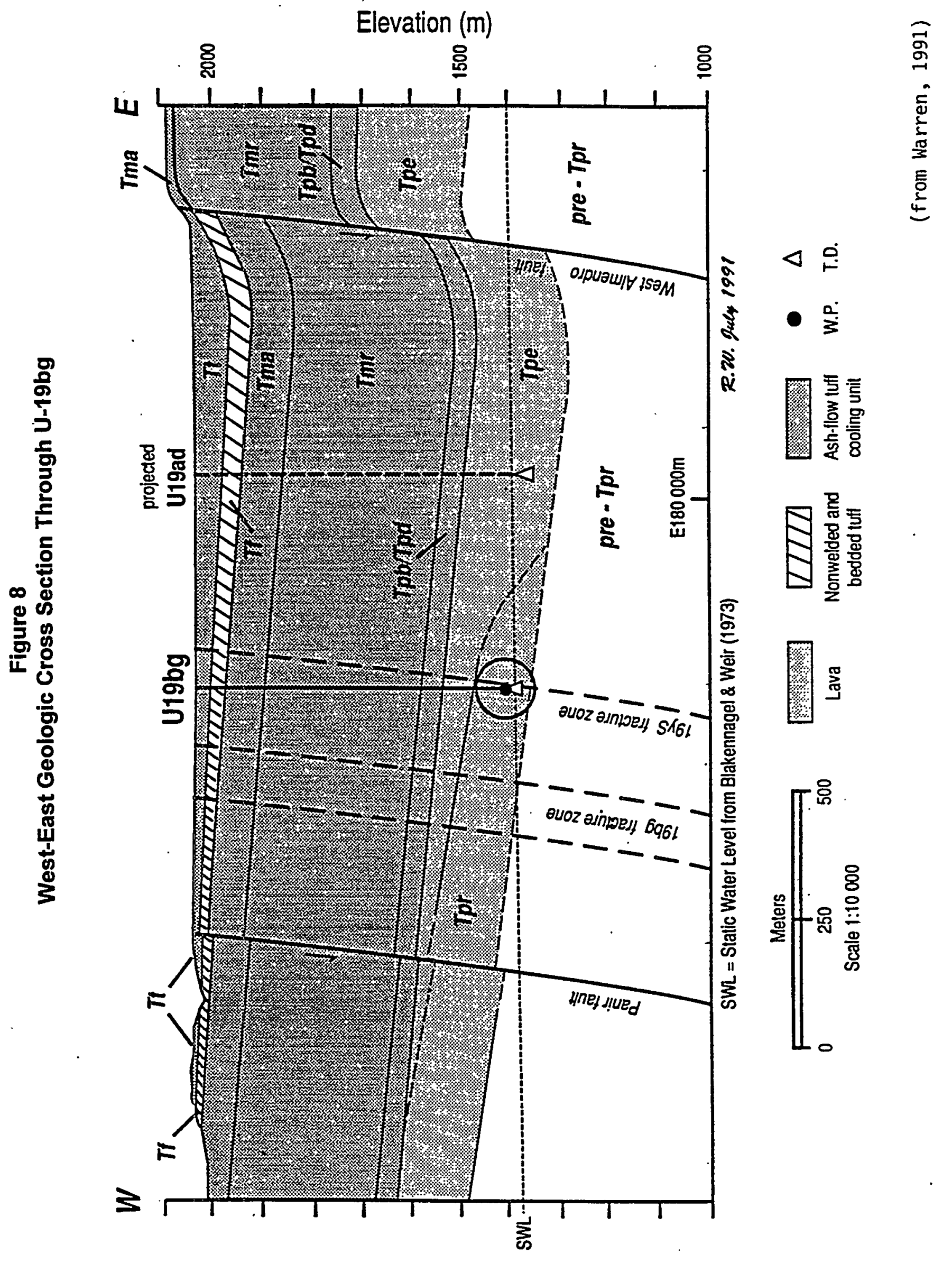




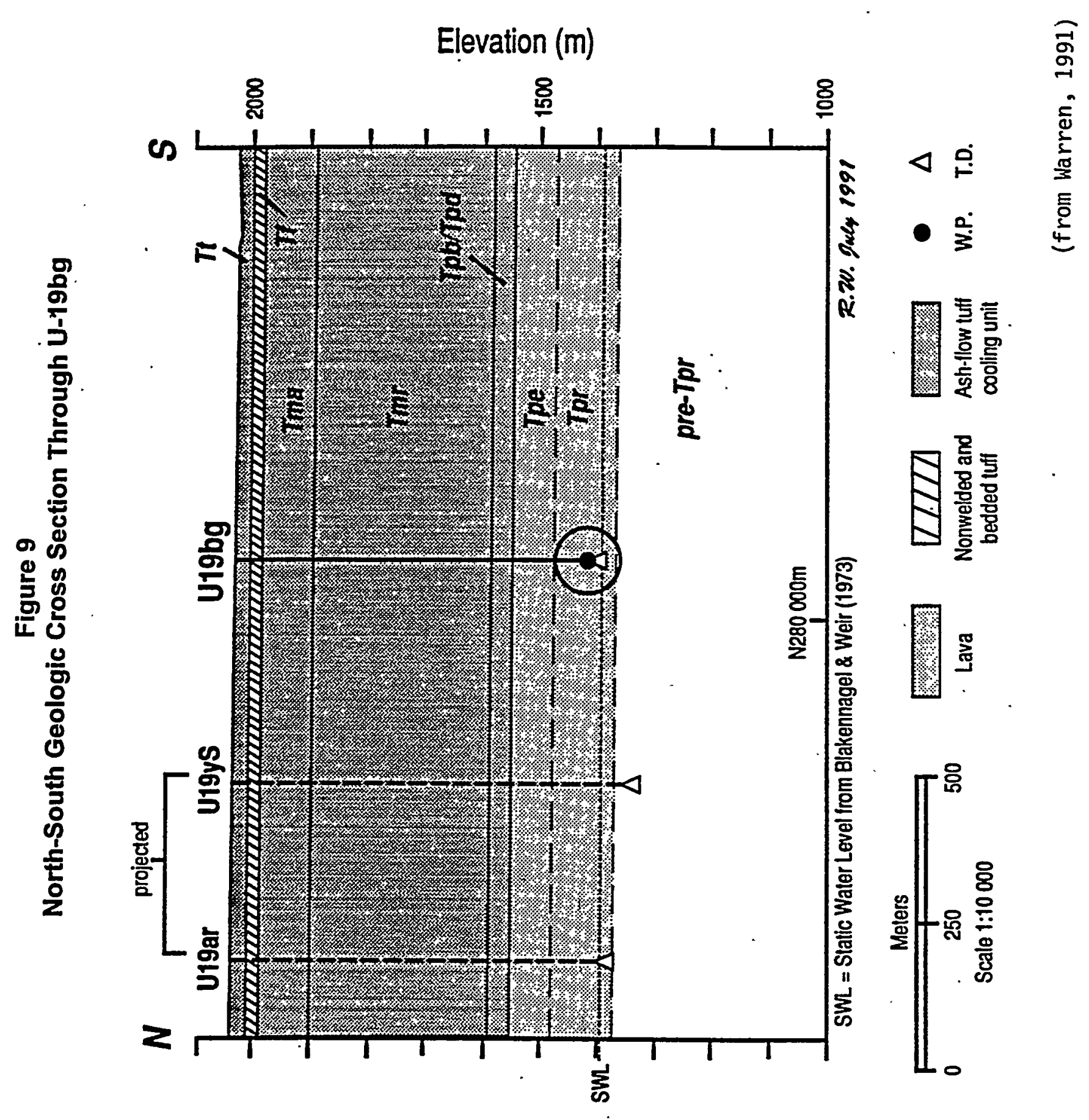


This page intentionally left blank. 
Stratigraphic Log of U-19bg Satatellite Hole \#1

Logged by Lance Prothro, Raytheon Services Nevada, August 1991

\begin{tabular}{|c|c|c|c|c|c|c|}
\hline \multicolumn{2}{|c|}{ Depth } & \multirow[t]{2}{*}{ Lithology } & \multirow[t]{2}{*}{ Stratigraphic Unit } & \multirow[t]{2}{*}{ Symbol } & \multicolumn{2}{|c|}{ Thickness } \\
\hline Meters & Feet & & & & Meters & Feet \\
\hline $0-9.1$ & $0-30$ & No samples. & $\begin{array}{l}\text { Thirsty Canyon Group, } \\
\text { undifferentiated }\end{array}$ & $\mathrm{Tt}$ & 9.1 & 30 \\
\hline $9.1-29.3$ & $30-96$ & $\begin{array}{l}\text { Ash-Flow Tuff: Partially } \\
\text { welded, devitrified. }\end{array}$ & $\begin{array}{l}\text { Pahute Mesa Tuff, } \\
\text { Thirsty Canyon Group }\end{array}$ & Ttp & 20.2 & 66 \\
\hline $29.3-35.5$ & $96-116.5$ & $\begin{array}{l}\text { Bedded Tuff: Vitric, partly } \\
\text { zeolitic, calcareous. }\end{array}$ & $\begin{array}{c}\text { rhyolite of } \\
\text { Chukar Canyon, } \\
\text { Volcanics of Fortymile } \\
\text { Canyon }\end{array}$ & Tfbr & 6.2 & 20.5 \\
\hline $35.5-49.4$ & $116.5-162$ & Bedded Tuff: Vitric. & $\begin{array}{c}\text { rhyolite of } \\
\text { Beatty Wash, } \\
\text { Volcanics of Fortymile } \\
\text { Canyon }\end{array}$ & Tfbw & 13.9 & 45.5 \\
\hline $49.4-51.2$ & $162-168$ & Bedded Tuff: Vitric. & $\begin{array}{l}\text { Ammonia Tanks Tuff } \\
\text { (bedded), } \\
\text { Timber Mountain Group }\end{array}$ & $\operatorname{Tma}(b)$ & 1.8 & 6 \\
\hline
\end{tabular}


Stratigraphic Log of U-19bg Satellite Hole \#1 (cont.)

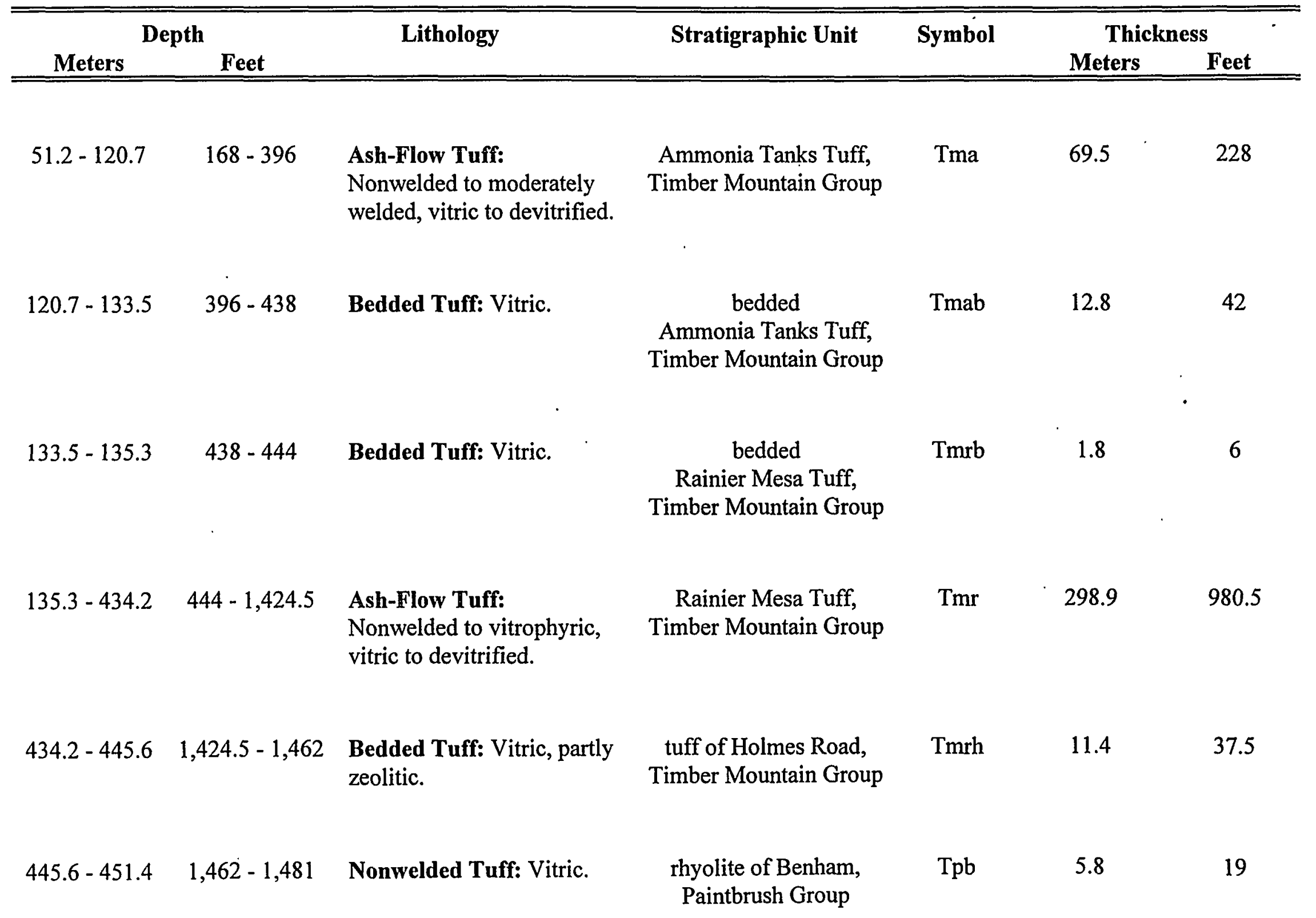




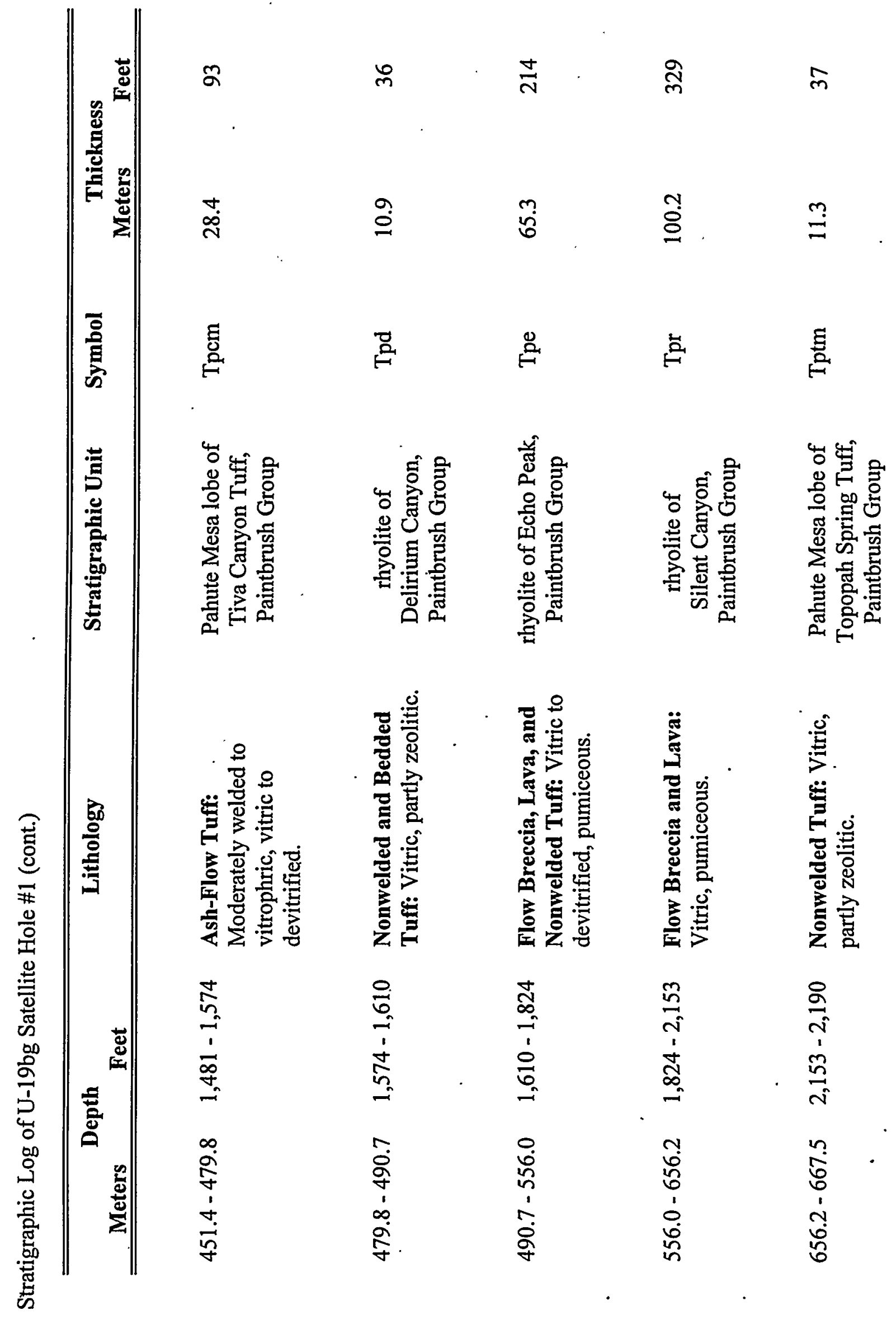


Stratigraphic Log of U-19bg Satellite Hole \#1 (cont.)

\begin{tabular}{|c|c|c|c|c|c|c|}
\hline \multicolumn{2}{|c|}{ Depth } & \multirow[t]{2}{*}{ Lithology } & \multirow[t]{2}{*}{ Stratigraphic Unit } & \multirow[t]{2}{*}{ Symból } & \multicolumn{2}{|c|}{ Thickness } \\
\hline Meters & Feet & & & & Meters & Feet \\
\hline $\begin{array}{c}667.5-685.8 \\
\text { TD }\end{array}$ & $\begin{array}{c}2,190-2,250 \\
\text { TD }\end{array}$ & Nonwelded Tuff: Vitric. & Calico Hills Formation & Th & $>18.3$ & $>60$ \\
\hline
\end{tabular}

NOTE: Due to the close proximity of this satellite hole to the U-19bg emplacement hole, only a cursory examination was made

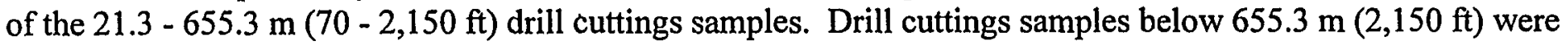
examined in detail. A detailed correlation of wireline geophysical logs for the entire borehole was conducted and the resultant formation tops are presented here. 


\section{Lithologic Log of U-19bg Satellite Hole \#1 \\ Logged by Lance Prothro, Raytheon Services Nevada \\ August 1991}

Unless otherwise noted, the following descriptions refer to washed cuttings samples at $3.05-\mathrm{m}$ (10-ft) intervals. Colors are determined by comparing wet sample color to the Geological Society of America Rock-Color Chart. Stratigraphic contacts and lithologic divisions are tied to geophysical logs whenever possible.

\section{Depth Meters/(feet) \\ Lithologic Description \\ Stratigraphic Unit}

NOTE: For detailed lithologic descriptions for the $0-656.2 \mathrm{~m}(0-2,153 \mathrm{ft})$ depth interval, see Emplacement Hole U-19bg.
$656.2-659.1 \mathrm{~m}$
$(2,153-2,162.5 \mathrm{ft})$
Nonwelded Tuff: Light-brown (5YR5/6); vitric; minor very-pale- orange and white pumice; minor felsic Pahute Mesa lobe of phenocrysts of feldspar; rare biotite; trace of clinopyroxene.
$659.1-667.5 \mathrm{~m}$
$(2,162.5-2,190 \mathrm{ft})$
Nonwelded Tuff: White to yellowish-gray; mostly vitric; partly zeolitic; silicified in part; minor white pumice; minor felsic phenocrysts of feldspar; rare biotite; trace of clinopyroxene; rare to minor moderate-reddish-brown silicified lithic fragments up to $10 \mathrm{~mm}$ in diameter and brownish-black subangular vitrophyre fragments up to $4 \mathrm{~mm}$ in diameter.
Nonwelded Tuff: Very-light-gray; vitric; minor white pumice; rare felsic phenocrysts of feldspar and quartz; trace of biotite. Topopah Spring Tuff
Pahute Mesa lobe of Topopah Spring Tuff
$682.8(?)->685.8 \mathrm{~m}$ $(2,240(?)->2,250 \mathrm{ft})$ TD
Nonwelded Tuff: Grayish-orange- pink; vitric; minor moderate-orange- pink pumice; minor felsic phenocrysts of feldspar and quartz; trace of biotite.
mafic poor Calico Hills Formation 


\section{U-19bg Satellite Hole \#1 Supplemental Data}

1. U-19bg Satellite Hole \#1 is located $11.0 \mathrm{~m}(36.1 \mathrm{ft})$ due south of Emplacement Hole U-19bg (Prothro, 1991f).

2. Bottom-hole measurements at U-19bg Sat \#1 on 01/31/1992 indicated a total depth of $672.4 \mathrm{~m}(2,206 \mathrm{ft})$ and a depth to fluid level at $645.6 \mathrm{~m}(2,118 \mathrm{ft})$. 
Stratigraphic Log of Emplacement Hole U-19bh

Logged by Lance Prothro, Raytheon Services Nevada, August 1995

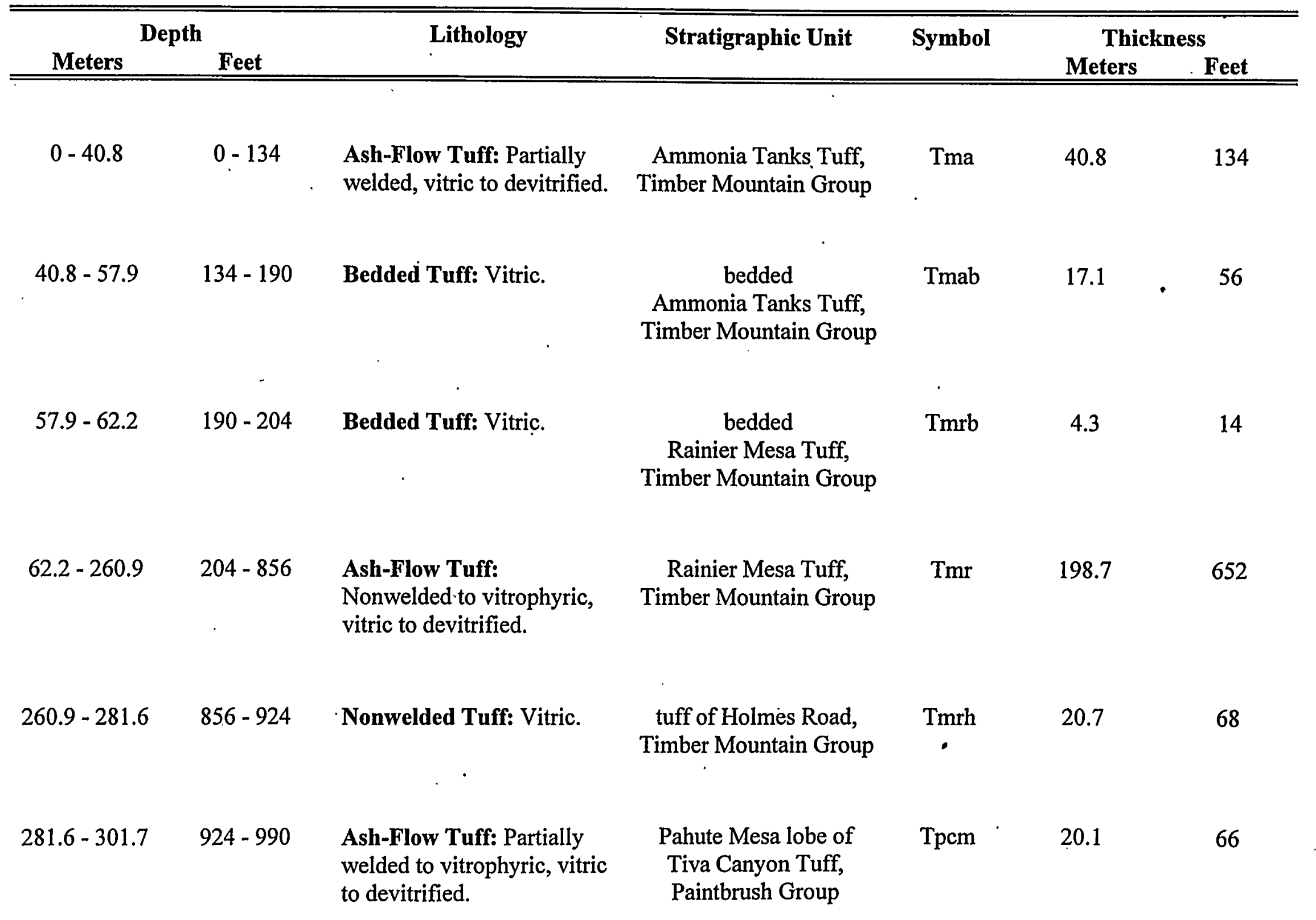


. Stratigraphic Log of Emplacement Hole U-19bh (cont.)

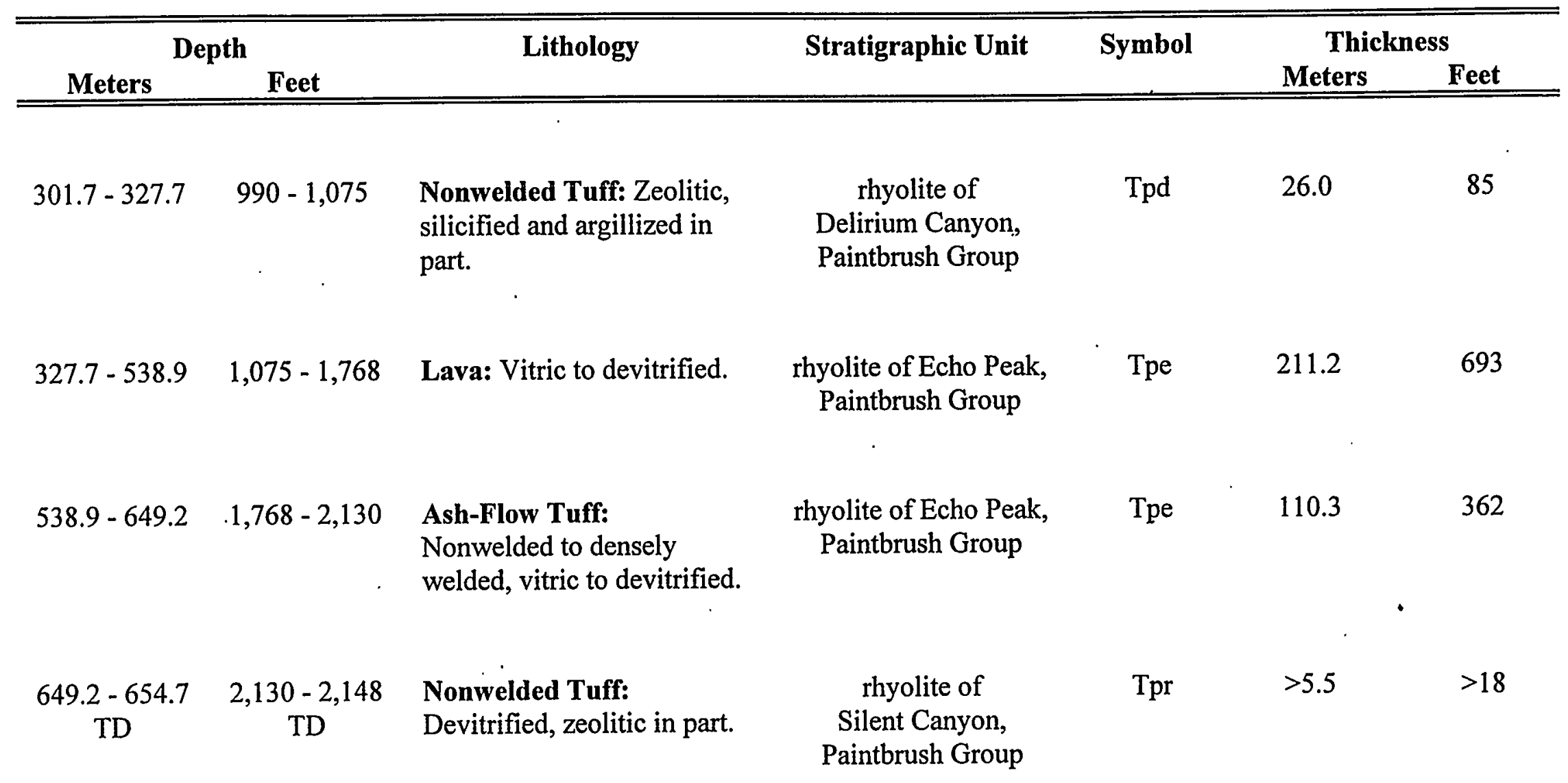




\section{Lithologic Log of Emplacement Hole U-19bh}

Logged by Lance Prothro, Raytheon Services Nevada

\section{August 1993}

Unless otherwise noted, the following descriptions refer to washed cuttings samples at $3.05-\mathrm{m}(10-\mathrm{ft})$ intervals. Colors are determined by comparing wet sample color to the Geological Society of America Rock-Color Chart. Stratigraphic contacts and lithologic divisions are tied to geophysical logs whenever possible. Geologic information gathered from the LLNL $16 \mathrm{~mm}$ film has been incorporated into the descriptions.

\begin{tabular}{|c|c|c|}
\hline $\begin{array}{c}\text { Depth } \\
\text { Meters/(feet) }\end{array}$ & Lithologic Description & Stratigraphic Unit \\
\hline $\begin{array}{l}0-21.3 \mathrm{~m} \\
(0-70 \mathrm{ft})\end{array}$ & Ash-Flow Tuff: No samples. & Ammonia Tanks Tuff \\
\hline $\begin{array}{c}21.3-32.3 \mathrm{~m} \\
(70-106 \mathrm{ft})\end{array}$ & $\begin{array}{l}\text { Partially Welded Ash-Flow Tuff: } \\
\text { Light-brown (5YR5/6); devitrified with } \\
\text { vapor-phase mineralization; common } \\
\text { pale-yellowish-orange pumice; } \\
\text { common felsic phenocrysts of feldspar, } \\
\text { including chatoyant sanidine, and } \\
\text { dipyramidal quartz; rare mafic minerals } \\
\text { of biotite with a trace of clinopyroxene } \\
\text { and hornblende; rare moderate-brown } \\
\text { lithic fragments less than } 3 \mathrm{~mm} \text { in } \\
\text { diameter; trace of sphene. }\end{array}$ & $\begin{array}{c}\text { mafic-poor } \\
\text { Ammonia Tanks Tuff }\end{array}$ \\
\hline $\begin{array}{l}32.3-40.8 \mathrm{~m} \\
(106-134 \mathrm{ft})\end{array}$ & $\begin{array}{l}\text { Partially Welded Ash-Flow Tuff: } \\
\text { Dark-yellowish-brown; vitric; minor } \\
\text { white and dusky-yellowish-brown } \\
\text { pumice; minor felsic phenocrysts of } \\
\text { feldspar, including chatoyant sanidine, } \\
\text { and quartz; rare mafic minerals of } \\
\text { biotite and clinopyroxene; rare } \\
\text { moderate-reddish-brown lithic } \\
\text { fragments less than } 2 \text { mm in diameter; } \\
\text { conspicuous black cuspate glass } \\
\text { shards; trace of sphene. }\end{array}$ & $\begin{array}{c}\text { mafic-poor } \\
\text { Ammonia Tanks Tuff }\end{array}$ \\
\hline
\end{tabular}


Lithologic Log of Emplacement Hole U-19bh (cont.)

Depth

Lithologic Description

Stratigraphic Unit

Meters/(feet)

$40.8-57.9 \mathrm{~m}$

$(134-190 \mathrm{ft})$
Bedded Tuff: Light-brownish-gray; vitric; common white to yellowish-gray pumice at $57.3 \mathrm{~m}(188 \mathrm{ft})$; common felsic phenocrysts of feldspar and quartz; rare to minor mafic minerals of biotite, clinopyroxene, and magnetite; minor grayish-brown lithic fragments less than $2 \mathrm{~mm}$ in diameter at $41.5 \mathrm{~m}$ (136 ft). Description is from percussion gun samples at $41.5 \mathrm{~m}$ (136ft) and $57.3 \mathrm{~m}(188 \mathrm{ft})$. Drill cuttings samples are unconsolidated mixtures consisting mainly of felsic crystals and pumice. Bedding contact at $44.8 \mathrm{~m}(147 \mathrm{ft})$ is approximately horizontal.

$57.9-62.2 \mathrm{~m}$ $(190-204 \mathrm{ft})$
Bedded Tuff: Grayish-orange to darkyellowish-orange; vitric; common white pumice; minor felsic phenocrysts of feldspar and quartz; minor mafic minerals of biotite and clinopyroxene. Percussion gun sample at $58.5 \mathrm{~m}$ $(192 \mathrm{ft}$ ) is partially zeolitic with abundant grayish-orange and grayishorange-pink pumice and minor bronze colored biotite.

bedded Ammonia Tanks Tuff 


\begin{tabular}{|c|c|c|}
\hline $\begin{array}{c}\text { Depth } \\
\text { Meters/(feet) }\end{array}$ & Lithologic Description & Stratigraphic Unit \\
\hline $\begin{array}{l}62.2-64.6 \mathrm{~m} \\
(204-212 \mathrm{ft})\end{array}$ & $\begin{array}{l}\text { Nonwelded Ash-Flow Tuff: Pale-red } \\
\text { (5R6/2) and light-brown ( } 5 \mathrm{YR} 5 / 6) ; \\
\text { partially devitrified with the pale-red } \\
\text { fragments showing a higher degree of } \\
\text { devitrification and some vapor-phase } \\
\text { mineralization; minor white and pale- } \\
\text { yellowish-orange vitric pumice; } \\
\text { common felsic phenocrysts of feldspar } \\
\text { and quartz; abundant biotite, trace of } \\
\text { orthopyroxene, trace of clinopyroxene } \\
\text { in percussion gun sample at } 63.1 \mathrm{~m} \\
\text { (207 ft); rare moderate-red ( } 5 \mathrm{R} 5 / 4) \\
\text { lithic fragments less than } 2 \text { mm in } \\
\text { diameter; rare pale-yellowish-orange } \\
\text { cuspate glass shards. }\end{array}$ & $\begin{array}{c}\text { mafic-rich } \\
\text { Rainier Mesa Tuff }\end{array}$ \\
\hline $\begin{array}{l}64.6-85.9 \mathrm{~m} \\
(212-282 \mathrm{ft})\end{array}$ & $\begin{array}{l}\text { Partially Welded Ash-Flow Tuff: } \\
\text { Dark-reddish-brown to grayish-red; } \\
\text { devitrified; minor light-gray pumice; } \\
\text { minor felsic phenocrysts of feldspar } \\
\text { and quartz; common mafic minerals of } \\
\text { biotite and clinopyroxene; rare } \\
\text { moderate-red (5R5/4) lithic fragments } \\
\text { less than } 3 \mathrm{~mm} \text { in diameter; numerous } \\
\text { pieces of botryoidal opal occur in } \\
\text { samples near base of interval. Poorly } \\
\text { developed fractures throughout } \\
\text { interval. }\end{array}$ & $\begin{array}{c}\text { mafic-rich } \\
\text { Rainier Mesa Tuff }\end{array}$ \\
\hline $\begin{array}{l}85.9-90.2 \mathrm{~m} \\
(282-296 \mathrm{ft})\end{array}$ & $\begin{array}{l}\text { Moderately Welded Ash-Flow Tuff: } \\
\text { Grayish-red; devitrified; common } \\
\text { white to pale-greenish-yellow pumice; } \\
\text { minor felsic phenocrysts of feldspar } \\
\text { and quartz; minor mafic minerals of } \\
\text { biotite and clinopyroxene. Prominent } \\
\text { fractures throughout interval. }\end{array}$ & $\begin{array}{c}\text { mafic-rich } \\
\text { Rainier Mesa Tuff }\end{array}$ \\
\hline
\end{tabular}




\begin{tabular}{|c|c|c|}
\hline $\begin{array}{c}\text { Depth } \\
\text { Meters/(feet) }\end{array}$ & Lithologic Description & Stratigraphic Unit \\
\hline $\begin{array}{l}90.2-245.1 \mathrm{~m} \\
(296-804 \mathrm{ft})\end{array}$ & $\begin{array}{l}\text { Moderately Welded Ash-Flow Tuff: } \\
\text { Grayish-red; devitrified; common } \\
\text { white to pale-greenish-yellow pumice; } \\
\text { minor felsic phenocrysts of feldspar } \\
\text { and quartz; rare biotite. Poorly } \\
\text { developed lithophysae from } 194.0 \text { - } \\
240.9 \mathrm{~m} \text { ( } 637 \text { - } 790 \mathrm{ft}) \text {. Moderately } \\
\text { developed lithophysae from } 240.9 \text { - } \\
245.1 \mathrm{~m} \text { ( } 790 \text { - } 804 \mathrm{ft}) \text {. Poorly } \\
\text { developed fractures from } 125.3 \text { - } \\
188.4 \mathrm{~m} \text { ( } 411 \text { - } 618 \mathrm{ft}) . \text { Moderately } \\
\text { developed fractures from } 117.0 \text { - } \\
125.3 \mathrm{~m} \text { ( } 384-411 \mathrm{ft}) \text {. Prominent } \\
\text { fractures from } 86.6 \text { - } 117.0 \mathrm{~m}(284 \text { - } \\
384 \mathrm{ft}) \text {. }\end{array}$ & $\begin{array}{c}\text { mafic-poor } \\
\text { Rainier Mesa Tuff }\end{array}$ \\
\hline $\begin{array}{c}245.1-249.0 \mathrm{~m} \\
(804-817 \mathrm{ft})\end{array}$ & $\begin{array}{l}\text { Vitrophyre: Brownish-black; vitric; } \\
\text { minor felsic phenocrysts of feldspar } \\
\text { and quartz; rare biotite; abundant clear } \\
\text { to black glass spherules. Poorly } \\
\text { developed fractures throughout } \\
\text { interval. }\end{array}$ & $\begin{array}{c}\text { mafic-poor } \\
\text { Rainier Mesa Tuff }\end{array}$ \\
\hline $\begin{array}{c}249.0-250.8 \mathrm{~m} \\
(817-823 \mathrm{ft})\end{array}$ & $\begin{array}{l}\text { Moderately Welded Ash-Flow Tuff: } \\
\text { Not represented in cuttings; lithology } \\
\text { inferred from geophysical logs. }\end{array}$ & $\begin{array}{c}\text { mafic-poor } \\
\text { Rainier Mesa Tuff }\end{array}$ \\
\hline $\begin{array}{c}250.8-252.1 \mathrm{~m} \\
(823-827 \mathrm{ft})\end{array}$ & $\begin{array}{l}\text { Partially Welded Ash-Flow Tuff: } \\
\text { Not represented in cuttings; lithology } \\
\text { inferred from geophysical logs. }\end{array}$ & $\begin{array}{c}\text { mafic-poor } \\
\text { Rainier Mesa Tuff }\end{array}$ \\
\hline
\end{tabular}


Lithologic Log of Emplacement Hole U-19bh (cont.)

\begin{tabular}{|c|c|c|}
\hline $\begin{array}{c}\text { Depth } \\
\text { Meters/(feet) }\end{array}$ & Lithologic Description & Stratigraphic Unit \\
\hline $\begin{array}{c}252.1-260.9 \mathrm{~m} \\
(827-856 \mathrm{ft})\end{array}$ & $\begin{array}{l}\text { Nonwelded Ash-Flow Tuff: Pale-red } \\
\text { (10R6/2); mostly vitric, devitrified in } \\
\text { part; minor felsic phenocrysts of } \\
\text { feldspar and lesser quartz; trace of } \\
\text { biotite; rare dusky-yellowish-brown } \\
\text { lithic fragments less than } 3 \mathrm{~mm} \text { in } \\
\text { diameter. Description is from } \\
\text { percussion gun sample at } 260.6 \mathrm{~m} \\
\text { ( } 855 \mathrm{ft} \text { ). Drill cuttings samples are } \\
\text { unconsolidated mixtures consisting } \\
\text { mainly of felsic crystals with lesser } \\
\text { amounts of pumice and lithic } \\
\text { fragments. Contact with underlying } \\
\text { Tmrh is approximately horizontal. }\end{array}$ & $\begin{array}{c}\text { mafic-poor } \\
\text { Rainier Mesa Tuff }\end{array}$ \\
\hline $\begin{array}{c}260.9-269.4 \mathrm{~m} \\
(856-884 \mathrm{ft})\end{array}$ & $\begin{array}{l}\text { Nonwelded Tuff: Light-brown } \\
\text { (5YR5/6); vitric; common white to } \\
\text { very-pale-orange pumice; minor felsic } \\
\text { phenocrysts of feldspar and quartz; } \\
\text { minor very small mafic minerals of } \\
\text { biotite and hornblende; common light- } \\
\text { brownish-gray to brownish-gray lithic } \\
\text { fragments less than } 5 \mathrm{~mm} \text { in diameter. }\end{array}$ & tuff of Holmes Road \\
\hline $\begin{array}{c}269.4-281.6 \mathrm{~m} \\
(884-924 \mathrm{ft})\end{array}$ & $\begin{array}{l}\text { Nonwelded Tuff: Grayish-orange- } \\
\text { pink ( } 5 \text { YR } 7 / 2) \text {; vitric; minor felsic } \\
\text { phenocrysts of quartz and lesser } \\
\text { feldspar; rare very small mafic } \\
\text { minerals of biotite and magnetite. } \\
\text { Description is from percussion gun } \\
\text { sample at } 281.3 \mathrm{~m} \text { ( } 923 \mathrm{ft} \text { ). Drill } \\
\text { cuttings samples are poor in quality } \\
\text { and consist mainly of pumice and lithic } \\
\text { fragments with a trace of sphene. }\end{array}$ & tuff of Holmes Road \\
\hline
\end{tabular}


Lithologic Log of Emplacement Hole U-19bh (cont.)

\begin{tabular}{|c|c|c|}
\hline $\begin{array}{c}\text { Depth } \\
\text { Meters/(feet) }\end{array}$ & Lithologic Description & Stratigraphic Unit \\
\hline $\begin{array}{c}281.6-290.8 \mathrm{~m} \\
(924-954 \mathrm{ft})\end{array}$ & $\begin{array}{l}\text { Vitrophyre: Brownish-black; vitric; } \\
\text { minor feldspar; rare mafic minerals of } \\
\text { biotite and clinopyroxene. Poorly } \\
\text { developed fractures throughout } \\
\text { interval. }\end{array}$ & $\begin{array}{l}\text { Pahute Mesa lobe of } \\
\text { Tiva Canyon Tuff }\end{array}$ \\
\hline $\begin{array}{c}290.8-296.9 \mathrm{~m} \\
(954-974 \mathrm{ft})\end{array}$ & $\begin{array}{l}\text { Densely Welded Ash-Flow Tuff: } \\
\text { Moderate-brown ( } 5 \text { YR } 4 / 4) \text {; devitrified; } \\
\text { rare feldspar; minor mafic minerals of } \\
\text { biotite and clinopyroxene. Poorly } \\
\text { developed fractures throughout } \\
\text { interval. }\end{array}$ & $\begin{array}{l}\text { Pahute Mesa lobe of } \\
\text { Tiva Canyon Tuff }\end{array}$ \\
\hline $\begin{array}{c}296.9-301.7 \mathrm{~m} \\
(974-990 \mathrm{ft})\end{array}$ & $\begin{array}{l}\text { Partially Welded Ash-Flow Tuff: } \\
\text { Dark-yellowish-brown; partially } \\
\text { devitrified with minor vapor-phase } \\
\text { mineralization; rare feldspar; minor } \\
\text { mafic minerals of biotite and } \\
\text { clinopyroxene; trace of dark-reddish- } \\
\text { brown lithic fragments less than } 2 \mathrm{~mm} \\
\text { in.diameter; common dusky-yellowish- } \\
\text { brown cuspate glass shards; trace of } \\
\text { sphene. }\end{array}$ & $\begin{array}{c}\text { Pahute Mesa lobe of } \\
\text { Tiva Canyon Tuff }\end{array}$ \\
\hline $\begin{array}{l}301.7-305.7 \mathrm{~m} \\
(990-1,003 \mathrm{ft})\end{array}$ & $\begin{array}{l}\text { Nonwelded Tuff: Sample quality is } \\
\text { poor. Pinkish-gray; partly vitric, partly } \\
\text { zeolitic; minor feldspar; rare mafic } \\
\text { minerals of biotite and hornblende; rare } \\
\text { pale-brown lithic fragments less than } \\
1 \mathrm{~mm} \text { in diameter; minor clear platy } \\
\text { glass shards; trace of sphene. }\end{array}$ & $\begin{array}{c}\text { rhyolite of } \\
\text { Delirium Canyon }\end{array}$ \\
\hline
\end{tabular}




\begin{tabular}{|c|c|c|}
\hline $\begin{array}{c}\text { Depth } \\
\text { Meters/(feet) }\end{array}$ & Lithologic Description & Stratigraphic Unit \\
\hline $\begin{array}{c}305.7-323.1 \mathrm{~m} \\
(1,003-1,060 \mathrm{ft})\end{array}$ & $\begin{array}{l}\text { Nonwelded Tuff: Samples consist } \\
\text { mainly of white and translucent pumice } \\
\text { and moderate-red ( } 5 \mathrm{R} 4 / 6) \text { and light- } \\
\text { brownish-gray to medium-light-gray } \\
\text { devitrified lava fragments. A thin, } \\
\text { white, vitric matrix coats some of the } \\
\text { pumice and lava fragments. Samples } \\
\text { also contain feldspar; trace of biotite } \\
\text { and clinopyroxene, clear glass } \\
\text { spherules and fragments, and a trace of } \\
\text { sphene. }\end{array}$ & $\begin{array}{c}\text { rhyolite of } \\
\text { Delirium Canyon }\end{array}$ \\
\hline $\begin{array}{c}323.1-327.7 \mathrm{~m} \\
(1,060-1,075 \mathrm{ft})\end{array}$ & $\begin{array}{l}\text { Nonwelded Tuff: ,Sample quality is } \\
\text { poor. Grayish-pink and pale-brown; } \\
\text { mostly zeolitic, partially vitric, } \\
\text { silicified and argillized in part; minor } \\
\text { very-pale-orange zeolitized pumice and } \\
\text { translucent vitric pumice; minor } \\
\text { feldspar; rare biotite, minor medium- } \\
\text { dark-gray lithic fragments less than } \\
3 \mathrm{~mm} \text { in diameter; trace of sphene. }\end{array}$ & $\begin{array}{c}\text { rhyolite of } \\
\text { Delirium Canyon }\end{array}$ \\
\hline $\begin{array}{c}327.7-332.2 \mathrm{~m} \\
(1,075-1,090 \mathrm{ft})\end{array}$ & $\begin{array}{l}\text { Flow Breccia: Medium-dark-gray; } \\
\text { poorly indurated; vitric; minor } \\
\text { feldspar; minor biotite; trace of sphene. } \\
\text { Poorly developed fractures throughout } \\
\text { interval. }\end{array}$ & rhyolite of Echo Peak \\
\hline $\begin{array}{c}332.2-339.8 \mathrm{~m} \\
(1,090-1,115 \mathrm{ft})\end{array}$ & $\begin{array}{l}\text { Lava: Medium-dark-gray; poorly } \\
\text { indurated; vitric; minor feldspar; minor } \\
\text { biotite; trace of sphene. Poorly } \\
\text { developed fractures throughout } \\
\text { interval. }\end{array}$ & rhyolite of Echo Peak \\
\hline
\end{tabular}


Lithologic Log of Emplacement Hole U-19bh (cont.)

\begin{tabular}{ccc}
\hline $\begin{array}{c}\text { Depth } \\
\text { Meters/(feet) }\end{array}$ & Lithologic Description & Stratigraphic Unit \\
\hline
\end{tabular}

$339.8-356.0 \mathrm{~m}$ $(1,115-1,168 \mathrm{ft})$

$356.0-420.0 \mathrm{~m}$ $(1168-1378 \mathrm{ft})$

$420.0-531.0 \mathrm{~m}$ $(1,378-1,742 \mathrm{ft})$

$531.0-538.9 \mathrm{~m}$ $(1,742-1,768 \mathrm{ft})$
Lava: Pale-yellowish-brown to darkyellowish-brown, mottled; moderately indurated; devitrified with vapor-phase mineralization producing concentric layers and conspicuous opaline botryoidal masses; minor feldspar; minor biotite, trace of clinopyroxene; numerous pale-red silicified fragments. Poorly developed fractures throughout interval.

Lava: Light-olive-gray and mediumlight gray; moderately indurated; vitric becoming partially devitrified below $398.1 \mathrm{~m}(1,306 \mathrm{ft})$; minor feldspar; minor biotite. Poorly developed fractures throughout interval.

Lava: Light-brownish-gray; moderately indurated; devitrified; minor feldspar, minor biotite, trace of clinopyroxene; trace of sphene. Flow banded from 444.8 - $449.6 \mathrm{~m}(1,459$ $1,475 \mathrm{ft})$. Poorly developed lithophysae from $414.5-444.7 \mathrm{~m}$ $(1,360-1,459 \mathrm{ft})$. Poorly developed fractures throughout interval.

Lava (basal vitrophyre): Brownishblack; vitric; minor feldspar; minor biotite; abundant black glass spherules. Poorly developed fractures throughout interval. rhyolite of Echo Peak

rhyolite of Echo Peak

rhyolite of Echo Peak

rhyolite of Echo Peak 
Lithologic Log of Emplacement Hole U-19bh (cont.)

\begin{tabular}{|c|c|c|}
\hline $\begin{array}{c}\text { Depth } \\
\text { Meters/(feet) }\end{array}$ & Lithologic Description & Stratigraphic Unit \\
\hline & . & . \\
\hline $\begin{array}{c}538.9-608.4 \mathrm{~m} \\
(1,768-1,996 \mathrm{ft})\end{array}$ & $\begin{array}{l}\text { Densely Welded Ash-Flow Tuff: } \\
\text { Pale-brown to moderate-brown } \\
\text { (5YR4/4); mostly devitrified with vitric } \\
\text { intervals from } 538.9-542.5 \mathrm{~m}(1,768 \text { - } \\
1,780 \mathrm{ft}), 581.2 \text { - } 581.6 \mathrm{~m}(1,907- \\
1,908 \mathrm{ft}) \text {, and } 583.0 \text { - } 585.6 \mathrm{~m}(1,913 \text { - } \\
1,921 \mathrm{ft}) \text {; minor moderate-reddish- } \\
\text { orange pumice below } 600.5 \mathrm{~m} \\
\text { (1,970 ft); minor feldspar; minor } \\
\text { biotite, trace of clinopyroxene; } \\
\text { common moderate-brown (5YR4/4) } \\
\text { lithic fragments less than } 5 \mathrm{~mm} \text { in } \\
\text { diameter; trace of sphene. Poorly } \\
\text { developed fractures to } 600.1 \mathrm{~m} \\
(1,969 \mathrm{ft}) . \text { Moderately developed } \\
\text { fractures below } 600.1 \mathrm{~m}(1,969 \mathrm{ft}) .\end{array}$ & rhyolite of Echo Peak \\
\hline $\begin{array}{c}608.4-626.1 \mathrm{~m} \\
(1,996-2,054 \mathrm{ft})\end{array}$ & $\begin{array}{l}\text { Densely Welded Ash-Flow Tuff: } \\
\text { Dark-yellowish-brown; vitric, } \\
\text { devitrified in part below } 621.8 \mathrm{~m} \\
(2,040 \mathrm{ft}) \text {; common black flattened } \\
\text { pumice; minor feldspar; rare biotite; } \\
\text { trace of sphene. Moderately developed } \\
\text { fractures throughout interval. }\end{array}$ & rhyolite of Echo Peak \\
\hline
\end{tabular}


Lithologic Log of Emplacement Hole U-19bh (cont.)

Depth

Lithologic Description

Stratigraphic Unit

Meters/(feet)

$626.1-635.8 \mathrm{~m}$

$(2,054-2,086 \mathrm{ft})$
Partially Welded Ash-Flow Tuff:

Pale-reddish-brown; devitrified, zeolitic in part; minor pale-yellowishorange to dark-yellowish-orange pumice; minor feldspar; minor biotite. Sample at $637.0 \mathrm{~m}(2,090 \mathrm{ft})$ contains abundant grayish-red and moderatereddish brown lithic fragments. These fragments are probably related to the interval of reversely graded, cobble- to boulder-size fragments observed on the $16 \mathrm{~mm}$ film from $632.0-635.0 \mathrm{~m}$ $(2,073.4-2,083.5 \mathrm{ft})$. Moderately developed fractures to $631.8 \mathrm{~m}$ $(2,073 \mathrm{ft})$.

$635.8-649.2 \mathrm{~m}$ $(2,086-2,130 \mathrm{ft})$
Nonwelded Ash-Flow Tuff: Lightbrown (5YR6/4); devitrified, zeolitic in part; common very-pale-orange pumice; minor feldspar; minor biotite, trace of clinopyroxene near base; minor brownish-gray to grayish-red (10R4/2) lithic fragments less than $8 \mathrm{~mm}$ in diameter; conspicuous sphene. Sample at $649.2 \mathrm{~m}(2,130 \mathrm{ft})$ is dominated by grayish-red (5R4/2) lithic(?) fragments. rhyolite of Echo Peak

rhyolite of Echo Peak 
Lithologic Log of Emplacement Hole U-19bh (cont.)

\begin{tabular}{clc}
\hline $\begin{array}{c}\text { Depth } \\
\text { Meters/(feet) }\end{array}$ & \multicolumn{1}{c}{ Lithologic Description } & Stratigraphic Unit \\
\hline $649.2-654.7 \mathrm{~m}$ & $\begin{array}{l}\text { Nonwelded Tuff: Moderate-reddish- } \\
\text { orange to moderate-reddish-brown; } \\
\text { devitrified, zeolitic in part; rare } \\
\text { grayish-orange pumice; common } \\
\text { feldspar; minor biotite (abundant } \\
\text { biotite in percussion gun sample at } \\
\text { TD }\end{array}$ & $\begin{array}{c}\text { rhyolite of } \\
\text { Silent Canyon }\end{array}$ \\
& $\begin{array}{l}\text { lithic fragments less than 1 mm in } \\
\text { diameter. Reddish color of sample is }\end{array}$ \\
& $\begin{array}{l}\text { the result of prevalent hematitic } \\
\text { staining. (Assignment of this interval } \\
\text { to Tpr is partly based on a cursory } \\
\text { examination of a grain mount of the } \\
\text { drill cuttings by R.G. Warren.) }\end{array}$
\end{tabular}




\section{Emplacement Hole U-19bh Supplemental Data}

1. For additional information regarding U-19bh, see Prothro (1995). Supplemental data listed below are also extracted from Prothro (1995).

2. LLNL 16-mm film and Sandia color video are available through BN Geology/Hydrology, Mercury, NV.

3. Percussion-gun samples are available from the interval $41.5-650.7 \mathrm{~m}(136-2,135 \mathrm{ft})$.

4. The borehole is significantly enlarged at approximately $315.8 \mathrm{~m}(1,036 \mathrm{ft})$. Prominent vertical fractures are present from $86.6-117.1 \mathrm{~m}(284-384 \mathrm{ft})$.

5. Fluid level measurements on $11 / 15 / 1994$ indicated a depth to fluid level of $638.6 \mathrm{~m}$ $(2,095.2 \mathrm{ft})$.

6. See graphic data summary sheet in back pocket. 

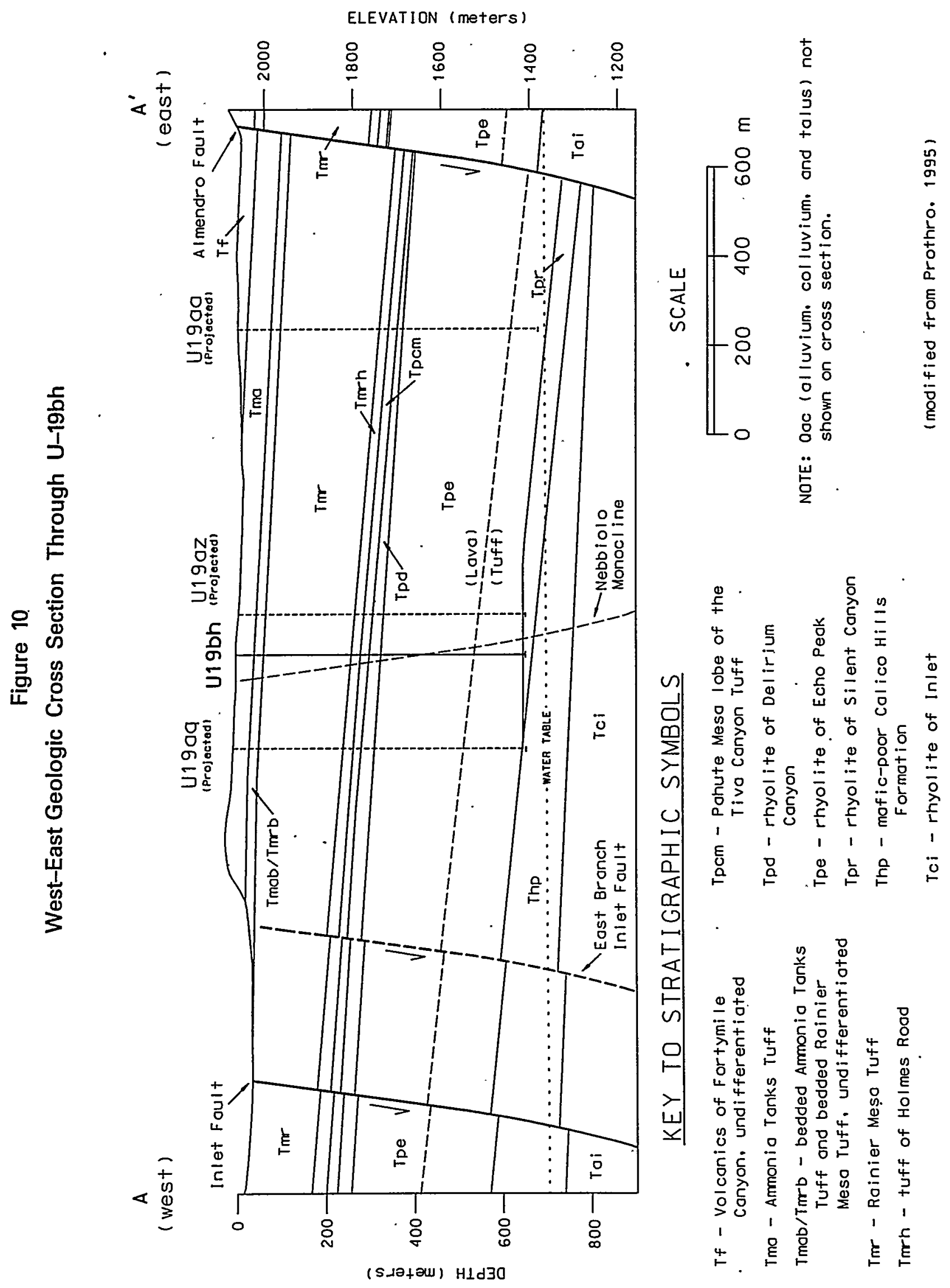

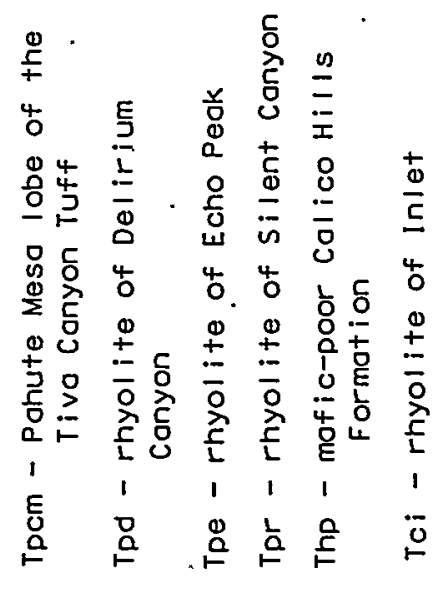

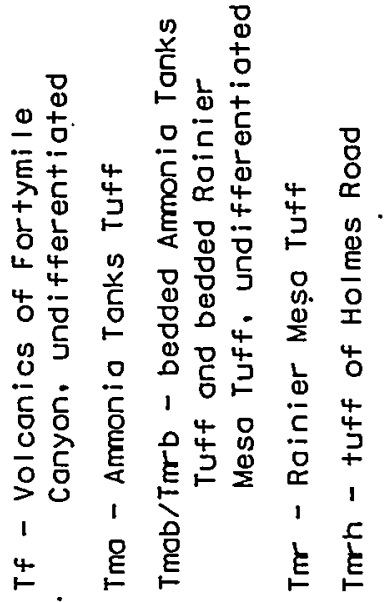



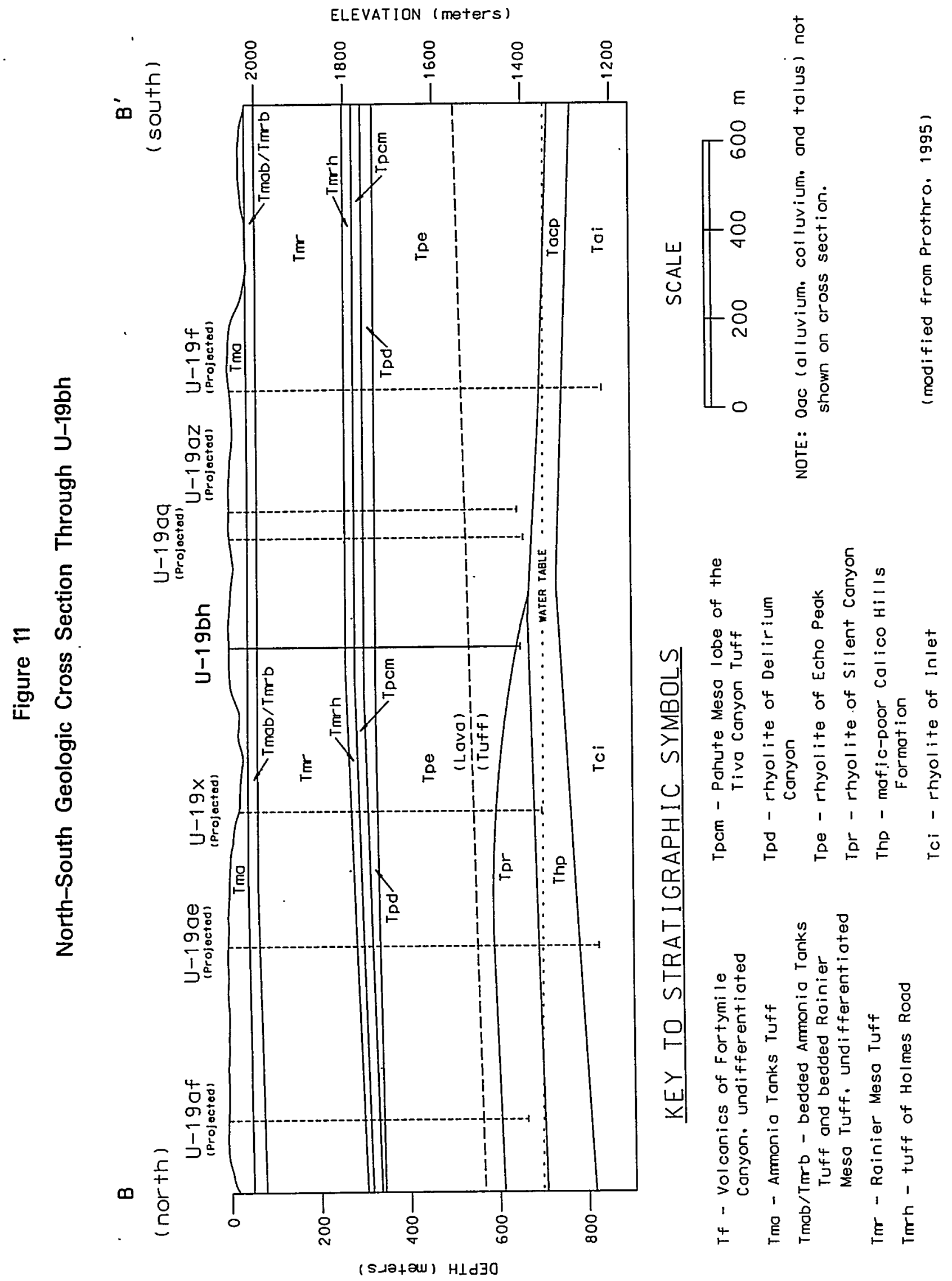
Stratigraphic Log of Emplacement Hole U-19bj

Logged by Lance Prothro, Bechtel Nevada, 10 July 1996

\begin{tabular}{|c|c|c|c|c|c|c|}
\hline \multirow[b]{2}{*}{ Meters } & Depth & \multirow[t]{2}{*}{ Lithology } & \multirow[t]{2}{*}{ Stratigraphic Unit } & \multirow[t]{2}{*}{ Symbol } & \multicolumn{2}{|c|}{ Thickness } \\
\hline & Feet & & & & Meters & Feet \\
\hline $0-64.6$ & $0-212$ & $\begin{array}{l}\text { Ash-Flow Tuff: } \\
\text { Nonwelded to densely } \\
\text { welded; mostly } \\
\text { devitrified. }\end{array}$ & $\begin{array}{c}\text { mafic-poor } \\
\text { Ammonia Tanks Tuff, } \\
\text { Timber Mountain Group }\end{array}$ & Tmap & 64.6 & 212 \\
\hline $64.6-73.5$ & $212-241$ & Bedded Tuff: Vitric. & $\begin{array}{c}\text { bedded } \\
\text { Ammonia Tanks Tuff, } \\
\text { Timber Mountain Group }\end{array}$ & Tmab & 8.9 & 29 \\
\hline $73.5-76.8$ & $241-252$ & Bedded Tuff: Vitric. & $\begin{array}{c}\text { bedded } \\
\text { Rainier Mesa Tuff, } \\
\text { Timber Mountain Group }\end{array}$ & Tmrb & 3.3 & 11 \\
\hline $76.8-108.2$ & $252-355$ & $\begin{array}{l}\text { Ash-Flow Tuff: } \\
\text { Nonwelded to } \\
\text { moderately welded; } \\
\text { vitric to devitrified. }\end{array}$ & $\begin{array}{c}\text { mafic-rich } \\
\text { Rainier Mesa Tuff, } \\
\text { Timber Mountain Group }\end{array}$ & Tmrr & 31.4 & 103 \\
\hline $108.2-367.9$ & $355-1,207$ & $\begin{array}{l}\text { Ash-Flow Tuff: } \\
\text { Nonwelded to } \\
\text { vitrophyric; vitric to } \\
\text { devitrified. }\end{array}$ & $\begin{array}{c}\text { mafic-poor } \\
\text { Rainier Mesa Tuff, } \\
\text { Timber Mountain Group }\end{array}$ & Tmrp & 259.7 & 852 \\
\hline
\end{tabular}


Stratigraphic Log of Emplacement Hole U-19bj (cont.)

\begin{tabular}{|c|c|c|c|c|c|c|}
\hline \multirow[b]{2}{*}{ Meters } & Depth & \multirow[t]{2}{*}{ Lithology } & \multirow[t]{2}{*}{ Stratigraphic Unit } & \multirow[t]{2}{*}{ Symbol } & \multicolumn{2}{|c|}{ Thickness } \\
\hline & Feet & & & & Meters & Feet \\
\hline $367.9-381.0$ & $1,207-1,250$ & Bedded Tuff: Vitric. & $\begin{array}{l}\text { tuff of Holmes Road, } \\
\text { Timber Mountain Group }\end{array}$ & Tmrh & 13.1 & 43 \\
\hline $381.0-509.0$ & $1,250-1,670$ & $\begin{array}{l}\text { Bedded Tuff, } \\
\text { Nonwelded Tuff, and } \\
\text { Pumiceous Lava: Vitric } \\
\text { to zeolitic. }\end{array}$ & $\begin{array}{c}\text { mafic-poor } \\
\text { Calico Hills Formation }\end{array}$ & Thp & 128.0 & 420 \\
\hline $\begin{array}{c}509.0-656.2 \\
\text { TD }\end{array}$ & $\begin{array}{c}1,670-2,153 \\
T D\end{array}$ & $\begin{array}{l}\text { Lava and Flow Breccia: } \\
\text { Vitric, devitrified, and } \\
\text { zeolitic; perlitic, } \\
\text { pumiceous, and } \\
\text { vitrophyric in part. }\end{array}$ & rhyolite of Kearsarge & Tcpk & $>147.2$ & $>483$ \\
\hline
\end{tabular}




\section{Lithologic Log of Emplacement Hole U-19bj \\ Logged by Lance Prothro, Bechtel Nevada \\ July 1996}

The lithologic descriptions follow Bechtel Nevada Department Procedure NTS-GEO-003, "Rock Sample Descriptions" (RSN, 1993). Stratigraphic and lithologic divisions are tied to geophysical logs whenever possible. Information utilized in the construction of the log included unpublished petrographic, lithologic, and stratigraphic data from R.G. Warren (LANL) and lithologic and stratigraphic data from Drellack (1990). Stratigraphic nomenclature is from Ferguson et al. (1994).

\begin{tabular}{|c|c|c|}
\hline $\begin{array}{c}\text { Depth } \\
\text { Meters/(feet) }\end{array}$ & Lithologic Description & Stratigraphic Unit \\
\hline $\begin{array}{l}0-21.3 \mathrm{~m} \\
(0-70 \mathrm{ft})\end{array}$ & $\begin{array}{l}\text { Partially Welded Ash-Flow Tuff: No } \\
\text { samples or geophysical logs available } \\
\text { for this interval. Geology inferred } \\
\text { from surface geology. }\end{array}$ & $\begin{array}{c}\text { mafic-poor } \\
\text { Ammonia Tanks Tuff }\end{array}$ \\
\hline $\begin{array}{l}21.3-41.1 \mathrm{~m} \\
(70-135 \mathrm{ft})\end{array}$ & $\begin{array}{l}\text { Partially Welded Ash-Flow Tuff: } \\
\text { Light-brownish-gray; partially } \\
\text { devitrified; minor white and moderate- } \\
\text { yellowish-brown pumice; minor felsic } \\
\text { phenocrysts of quartz and feldspar } \\
\text { including conspicuous chatoyant } \\
\text { sanidine; rare mafic minerals of biotite } \\
\text { and lesser clinopyroxene; trace of } \\
\text { sphene. }\end{array}$ & $\begin{array}{c}\text { mafic-poor } \\
\text { Ammonia Tanks Tuff }\end{array}$ \\
\hline $\begin{array}{l}41.1-50.9 \mathrm{~m} \\
(135-167 \mathrm{ft})\end{array}$ & $\begin{array}{l}\text { Moderately Welded Ash-Flow Tuff: } \\
\text { Brownish-gray; devitrified; minor } \\
\text { felsic phenocrysts of quartz and } \\
\text { feldspar including chatoyant sanidine; } \\
\text { rare mafic minerals of biotite and lesser } \\
\text { clinopyroxene; trace of sphene. A few } \\
\text { fragments of white chalcedony are } \\
\text { present in samples. }\end{array}$ & $\begin{array}{c}\text { mafic-poor } \\
\text { Ammonia Tanks Tuff }\end{array}$ \\
\hline
\end{tabular}


Lithologic Log of Emplacement Hole U-19bj (cont.)

Depth

Meters/(feet)

$50.9-59.1 \mathrm{~m}$

$(167-194 \mathrm{ft})$

$59.1-63.1 \mathrm{~m}$

$(194-207 \mathrm{ft})$

$63.1-64.6 \mathrm{~m}$

$(207-212 \mathrm{ft})$
Lithologic Description

Densely Welded Ash-Flow Tuff:

Brownish-gray; devitrified; minor

felsic phenocrysts of quartz and

feldspar; rare mafic minerals of biotite

and lesser clinopyroxene; trace of

sphene. A few fragments of white

chalcedony are present in samples.

Moderately Welded Ash-Flow Tuff:

Brownish-gray; devitrified; minor

felsic phenocrysts of quartz and

feldspar; rare mafic minerals of biotite

and lesser clinopyroxene; trace of

sphene. A few fragments of white

chalcedony are present in samples.

Partially Welded to Nonwelded AshFlow Tuff: Sample consists of loose felsic crystals of quartz and feldspar, white and moderate-orange-pink (5YR8/4) vitric pumice fragments, tuffaceous rock fragments, and mafic minerals of biotite and clinopyroxene. Lithology is partly based on geophysical logs.
Stratigraphic Unit

mafic-poor

Ammonia Tanks Tuff mafic-poor
Ammonia Tanks Tuff

mafic-poor

Ammonia Tanks Tuff 


\begin{tabular}{|c|c|}
\hline $\begin{array}{c}\text { Depth } \\
\text { Meters/(feet) }\end{array}$ & Lithologic Description \\
\hline $\begin{array}{l}64.6-73.5 \mathrm{~m} \\
(212-241 \mathrm{ft})\end{array}$ & $\begin{array}{l}\text { Bedded Tuff: Samples consist of } \\
\text { loose felsic crystals of quartz and } \\
\text { feldspar; mafic minerals of biotite, } \\
\text { clinopyroxene and lesser } \\
\text { orthopyroxene; white to light-brown } \\
\text { (5YR5/6) vitric pumice containing rare } \\
\text { felsic phenocrysts of feldspar and } \\
\text { quartz, and a trace of biotite, } \\
\text { clinopyroxene and sphene; and } \\
\text { tuffaceous rock fragments. }\end{array}$ \\
\hline
\end{tabular}

$73.5-76.8 \mathrm{~m}$ (241 - $252 \mathrm{ft})$

$76.8-81.7 \mathrm{~m}$ $(252-268 \mathrm{ft})$

$81.7-95.4 \mathrm{~m}$ $(268-313 \mathrm{ft})$
Bedded Tuff: Interval not recognized in samples. Lithology inferred from geophysical logs.

Nonwelded Tuff: Moderateyellowish-brown; vitric; common white pumice; minor felsic phenocrysts of quartz and feldspar; minor mafic minerals of biotite and lesser clinopyroxene and orthopyroxene; rare grayish-black lithic fragments less than $3 \mathrm{~mm}$ in diameter.

\section{Partially Welded Ash-Flow Tuff:}

Pale-reddish-brown to grayish-red (10R4/2); devitrified with minor vaporphase mineralization; rare very-paleorange pumice; rare felsic phenocrysts of quartz and lesser feldspar; minor mafic minerals of biotite and much less clinopyroxene; rare moderate-red lithic fragments less than $2 \mathrm{~mm}$ in diameter.
Stratigraphic Unit bedded .

Rainier Mesa Tuff

mafic-rich

Rainier Mesa tuff

mafic-rich Rainier Mesa Tuff 
Lithologic Log of Emplacement Hole U-19bj (cont.)

\begin{tabular}{|c|c|c|}
\hline $\begin{array}{c}\text { Depth } \\
\text { Meters/(feet) }\end{array}$ & Lithologic Description & Stratigraphic Unit \\
\hline $\begin{array}{c}95.4-108.2 \mathrm{~m} \\
(313-355 \mathrm{ft})\end{array}$ & $\begin{array}{l}\text { Moderately Welded Ash-Flow Tuff: } \\
\text { Pale-reddish-brown to grayish-red } \\
\text { (10R4/2); devitrified; rare white to } \\
\text { very-light-brownish-gray pumice; } \\
\text { minor felsic phenocrysts of feldspar } \\
\text { and quartz; minor mafic minerals of } \\
\text { biotite and much less clinopyroxene. } \\
\text { Interval approaches densely welded } \\
\text { and becomes partially devitrified from } \\
98.8 \text { - } 100.3 \mathrm{~m} \text { ( } 324 \text { - } 329 \mathrm{ft}) \text {. }\end{array}$ & $\begin{array}{c}\text { mafic-rich } \\
\text { Rainier Mesa Tuff }\end{array}$ \\
\hline $\begin{array}{l}108.2-332.8 \mathrm{~m} \\
(355-1,092 \mathrm{ft})\end{array}$ & $\begin{array}{l}\text { Moderately Welded Ash-Flow Tuff: } \\
\text { Grayish-red (10R4/2) to pinkish-gray, } \\
\text { mottled; devitrified; minor pinkish- } \\
\text { gray pumice; minor felsic phenocrysts } \\
\text { of feldspar and quartz; rare biotite; rare } \\
\text { grayish-red lithic fragments less than } \\
2 \mathrm{~mm} \text { in diameter. Interval becomes } \\
\text { densely welded and partially devitrified } \\
\text { from } 120.7 \text { to } 132.0 \mathrm{~m} \text { ( } 396 \text { - } 433 \mathrm{ft}) \\
\text { and at base below approximately } \\
329.2 \mathrm{~m}(1,080 \mathrm{ft}) \text {. }\end{array}$ & $\begin{array}{c}\text { mafic-poor } \\
\text { Rainier Mesa Tuff }\end{array}$ \\
\hline $\begin{array}{c}332.8-340.2 \mathrm{~m} \\
(1,092-1,116 \mathrm{ft})\end{array}$ & $\begin{array}{l}\text { Vitrophyre: Brownish-black to } \\
\text { grayish-black; vitric; minor felsic } \\
\text { phenocrysts of quartz and feldspar; rare } \\
\text { biotite. }\end{array}$ & $\begin{array}{c}\text { mafic-poor } \\
\text { Rainier Mesa Tuff }\end{array}$ \\
\hline $\begin{array}{c}340.2-343.2 \mathrm{~m} \\
(1,116-1,126 \mathrm{ft})\end{array}$ & $\begin{array}{l}\text { Densely Welded Ash-Flow Tuff: } \\
\text { Light-brownish-gray; vitric; minor } \\
\text { felsic phenocrysts of feldspar and } \\
\text { quartz; rare biotite. }\end{array}$ & $\begin{array}{c}\text { mafic-poor } \\
\text { Rainier Mesa Tuff }\end{array}$ \\
\hline
\end{tabular}


Lithologic Log of Emplacement Hole U-19bj (cont.)

\begin{tabular}{|c|c|c|}
\hline $\begin{array}{c}\text { Depth } \\
\text { Meters/(feet) }\end{array}$ & Lithologic Description & Stratigraphic Unit \\
\hline $\begin{array}{c}343.2-346.3 \mathrm{~m} \\
(1,126-1,136 \mathrm{ft})\end{array}$ & $\begin{array}{l}\text { Moderately Welded Ash-Flow Tuff: } \\
\text { Moderate-reddish-brown; vitric; minor } \\
\text { felsic phenocrysts of feldspar and } \\
\text { quartz; rare biotite; common pale- } \\
\text { yellowish-brown glass shards. }\end{array}$ & $\begin{array}{c}\text { mafic-poor } \\
\text { Rainier Mesa Tuff }\end{array}$ \\
\hline $\begin{array}{c}346.3-349.0 \mathrm{~m} \\
(1,136-1,145 \mathrm{ft})\end{array}$ & $\begin{array}{l}\text { Partially Welded Ash-Flow Tuff: } \\
\text { Moderate-reddish-orange; vitric; minor } \\
\text { white pumice; minor felsic phenocrysts } \\
\text { of quartz and feldspar; rare biotite; } \\
\text { common dusky-yellowish-brown glass } \\
\text { shards. }\end{array}$ & $\begin{array}{c}\text { mafic-poor } \\
\text { Rainier Mesa Tuff }\end{array}$ \\
\hline $\begin{array}{c}349.0-367.9 \mathrm{~m} \\
(1,145-1,207 \mathrm{ft})\end{array}$ & $\begin{array}{l}\text { Nonwelded Ash-Flow Tuff: Samples } \\
\text { consist of loose felsic crystals of quartz } \\
\text { and feldspar, fragments of white vitric } \\
\text { pumice, and moderate-yellowish- } \\
\text { brown glass shards. A moderate- } \\
\text { reddish-orange vitric matrix coats } \\
\text { many of the constituents of the } \\
\text { samples. Lithic fragments and loose } \\
\text { biotite flakes are present, but in much } \\
\text { less quantity than the above } \\
\text { constituents. }\end{array}$ & $\begin{array}{c}\text { mafic-poor } \\
\text { Rainier Mesa Tuff }\end{array}$ \\
\hline $\begin{array}{c}367.9-381.0 \mathrm{~m} \\
(1,207-1,250 \mathrm{ft})\end{array}$ & $\begin{array}{l}\text { Bedded Tuff: Light-brown ( } 5 \text { YR } 5 / 6) \text {; } \\
\text { vitric; rare to common pumice; minor } \\
\text { felsic phenocrysts of quartz and } \\
\text { feldspar; rare very small mafic } \\
\text { minerals of biotite, clinopyroxene and } \\
\text { hornblende; minor various colored } \\
\text { lithic fragments up to } 3 \mathrm{~mm} \text { in } \\
\text { diameter; trace of sphene; rare to } \\
\text { common glass shards. }\end{array}$ & tuff of Holmes Road \\
\hline
\end{tabular}


Lithologic Log of Emplacement Hole U-19bj (cont.)

\begin{tabular}{|c|c|c|}
\hline $\begin{array}{c}\text { Depth } \\
\text { Meters/(feet) }\end{array}$ & Lithologic Description & Stratigraphic Unit \\
\hline \multirow[t]{2}{*}{$\begin{array}{c}381.0-410.0 \mathrm{~m} \\
(1,250-1,345 \mathrm{ft})\end{array}$} & $\begin{array}{l}\text { Pumiceous Lava: White to very-light- } \\
\text { brownish-gray; vitric; rare felsic } \\
\text { phenocrysts of quartz and feldspar; rare } \\
\text { biotite. Samples from upper portion of } \\
\text { interval from approximately } 381.0 \text { - } \\
393.2 \mathrm{~m} \text { ( } 1,250 \text { - } 1,290 \mathrm{ft}) \text { consist of } \\
\text { loose felsic crystals of feldspar and } \\
\text { quartz, various lithic fragments } \\
\text { including pumiceous and devitrified } \\
\text { lava fragments, and loose mafic } \\
\text { minerals of biotite and clinopyroxene. } \\
\text { Vitric and devitrified Rainier Mesa } \\
\text { ash-flow tuff and vitrophyre fragments } \\
\text { from uphole are also present in } \\
\text { samples. }\end{array}$ & $\begin{array}{c}\text { mafic-poor } \\
\text { Calico Hills Formation }\end{array}$ \\
\hline & $\begin{array}{l}\text { The upper portion of this interval may } \\
\text { be a bedded tuff (tephra envelope) } \\
\text { overlying pumiceous lava. }\end{array}$ & \\
\hline $\begin{array}{c}410.0-430.4 \mathrm{~m} \\
(1,345-1,412 \mathrm{ft})\end{array}$ & $\begin{array}{l}\text { Bedded Tuff: Grayish-orange, } \\
\text { becoming grayish-yellow below } \\
\text { approximately } 426.7 \mathrm{~m}(1,400 \mathrm{ft}) \text {; } \\
\text { vitric to partially zeolitic, becoming } \\
\text { zeolitic and partially silicified below } \\
\text { approximately } 426.7 \mathrm{~m}(1,400 \mathrm{ft}) \text {; } \\
\text { abundant grayish-orange pumice; rare } \\
\text { to minor felsic phenocrysts of quartz } \\
\text { and feldspar; rare biotite; trace of } \\
\text { hornblende; minor to common grayish- } \\
\text { red ( } 5 \mathrm{R} 4 / 2) \text { lithic fragments (rhyolite } \\
\text { lava) up to } 10 \text { mm in diameter; } \\
\text { common botryoidal chalcedony } \\
\text { present, particularly as coatings on } \\
\text { lithic fragments, below approximately } \\
426.7 \mathrm{~m} \text { ( } 1,400 \mathrm{ft} \text { ). }\end{array}$ & $\begin{array}{l}\text { mafic-poor } \\
\text { Calico Hills Formation }\end{array}$ \\
\hline
\end{tabular}




\begin{tabular}{|c|c|c|}
\hline $\begin{array}{c}\text { Depth } \\
\text { Meters/(feet) }\end{array}$ & Lithologic Description & Stratigraphic Unit \\
\hline $\begin{array}{c}430.4-470.9 \mathrm{~m} \\
(1,412-1,545 \mathrm{ft})\end{array}$ & $\begin{array}{l}\text { Nonwelded Tuff: Dark-yellowish- } \\
\text { orange, becoming grayish-orange } \\
\text { towards base of interval; zeolitic; rare } \\
\text { felsic phenocrysts of quartz and } \\
\text { feldspar; rare biotite; rare moderate- } \\
\text { brown ( } 5 \text { YR4/4) lithic fragments up to } \\
3 \text { mm in diameter, becoming minor in } \\
\text { abundance and up to } 7 \mathrm{~mm} \text { in diameter } \\
\text { below approximately } 457.2 \mathrm{~m} \\
\text { (1,500 ft). }\end{array}$ & $\begin{array}{l}\text { mafic-poor } \\
\text { Calico Hills Formation }\end{array}$ \\
\hline $\begin{array}{c}470.9-509.0 \mathrm{~m} \\
(1,545-1,670 \mathrm{ft})\end{array}$ & $\begin{array}{l}\text { Bedded Tuff: 'Very-pale-orange, } \\
\text { grayish-yellow, and yellowish-gray; } \\
\text { mostly zeolitic, vitric in part; rare felsic } \\
\text { phenocrysts of feldspar and quartz; rare } \\
\text { biotite; rare to common mostly dark- } \\
\text { reddish-brown lithic fragments up to } \\
5 \mathrm{~mm} \text { in diameter. }\end{array}$ & $\begin{array}{c}\text { mafic-poor } \\
\text { Calico Hills Formation }\end{array}$ \\
\hline $\begin{array}{c}509.0-533.4 \mathrm{~m} \\
(1,670-1,750 \mathrm{ft})\end{array}$ & $\begin{array}{l}\text { Lava: Grayish-yellow to moderate- } \\
\text { yellow; zeolitic, vitric and perlitic in } \\
\text { part, becoming pumiceous towards } \\
\text { base of interval; minor feldspar } \\
\text { phenocrysts; abundant mafic minerals } \\
\text { of biotite and lesser clinopyroxene. }\end{array}$ & rhyolite of Kearsarge \\
\hline
\end{tabular}


Lithologic Log of Emplacement Hole U-19bj (cont.)

\begin{tabular}{ccc}
\hline $\begin{array}{c}\text { Depth } \\
\text { Meters/(feet) }\end{array}$ & Lithologic Description & Stratigraphic Unit \\
\hline
\end{tabular}

533.4-553.5 m Flow Breccia: Dusky-yellow and

$(1,750-1,816 \mathrm{ft}) \quad$ grayish-red (10R4/2); zeolitic and devitrified, pumiceous in part; minor feldspar phenocrysts; abundant mafic minerals of biotite and lesser clinopyroxene; common botryoidal chalcedony. Interval consists of grayish-red (10R4/2) devitrified lava fragments in a dusky-yellow, zeolitic, pumiceous matrix.

$553.5-653.8 \mathrm{~m}$

Lava: Pale-red (10R6/2) to grayish$(1,816-2,145 \mathrm{ft})$ red.(10R4/2); devitrified to partially devitrified to $609.6 \mathrm{~m}(2,000 \mathrm{ft})$, becoming partially zeolitic below; common feldspar phenocrysts; abundant mafic minerals of biotite and lesser clinopyroxene.

$653.8-656.2 \mathrm{~m}$

Vitrophyric Lava: Dusky-yellowish-

rhyolite of Kearsarge $(2,145-2,153 \mathrm{ft})$ TD brown and grayish-black; vitric; minor feldspar phenocrysts; common biotite.

rhyolite of Kearsarge

rhyolite of Kearsarge

ryolite of Kearsarge 


\section{Emplacement Hole U-19bj Supplemental Data}

1. Sandia color video available (Prothro, 1992).

2. Bottom-hole measurements on $07 / 18 / 1994$ indicated a depth to fluid level of $650.7 \mathrm{~m}$ $(2,135 \mathrm{ft})$ and a total depth of $655.3 \mathrm{~m}(2,150 \mathrm{ft})$. 


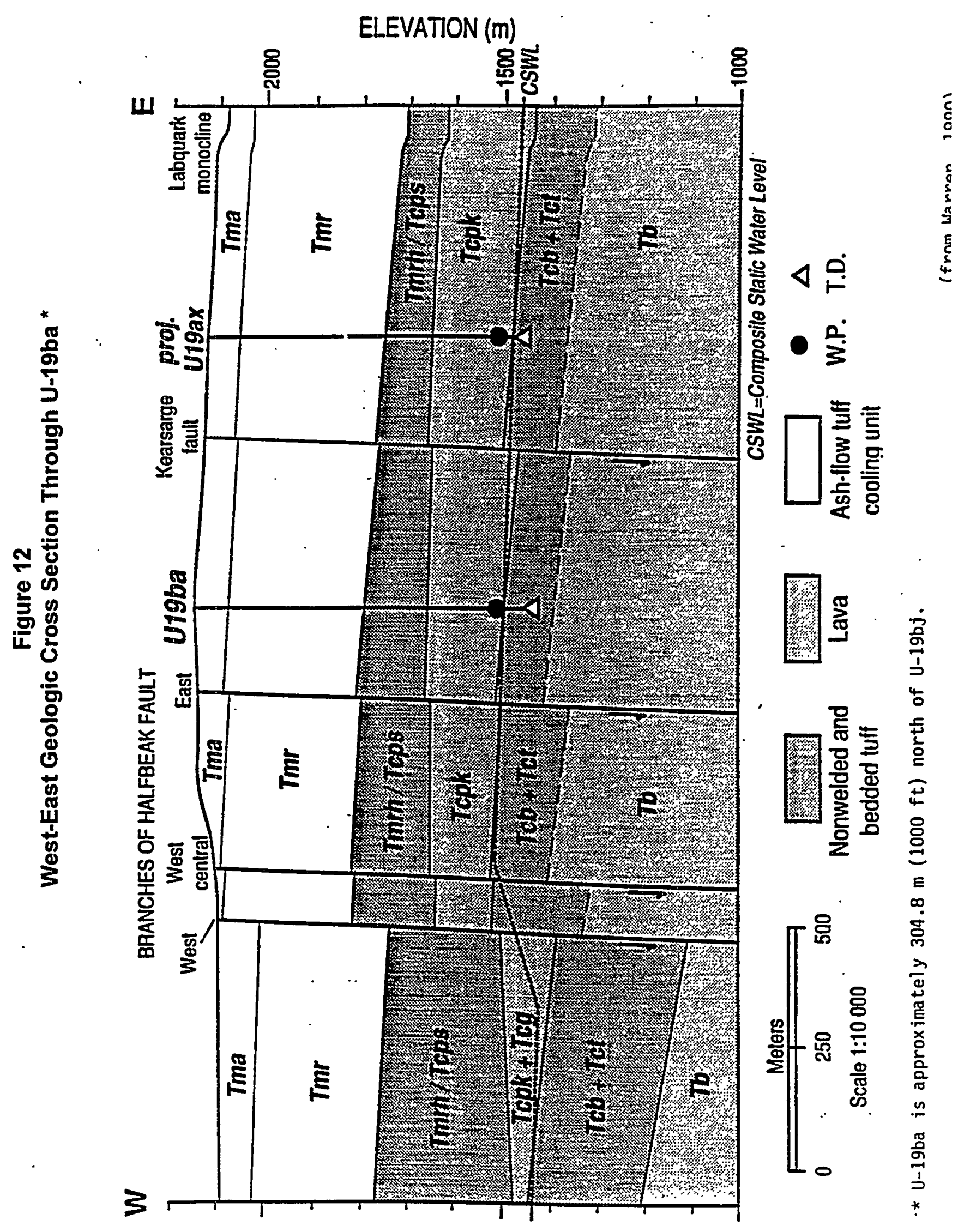



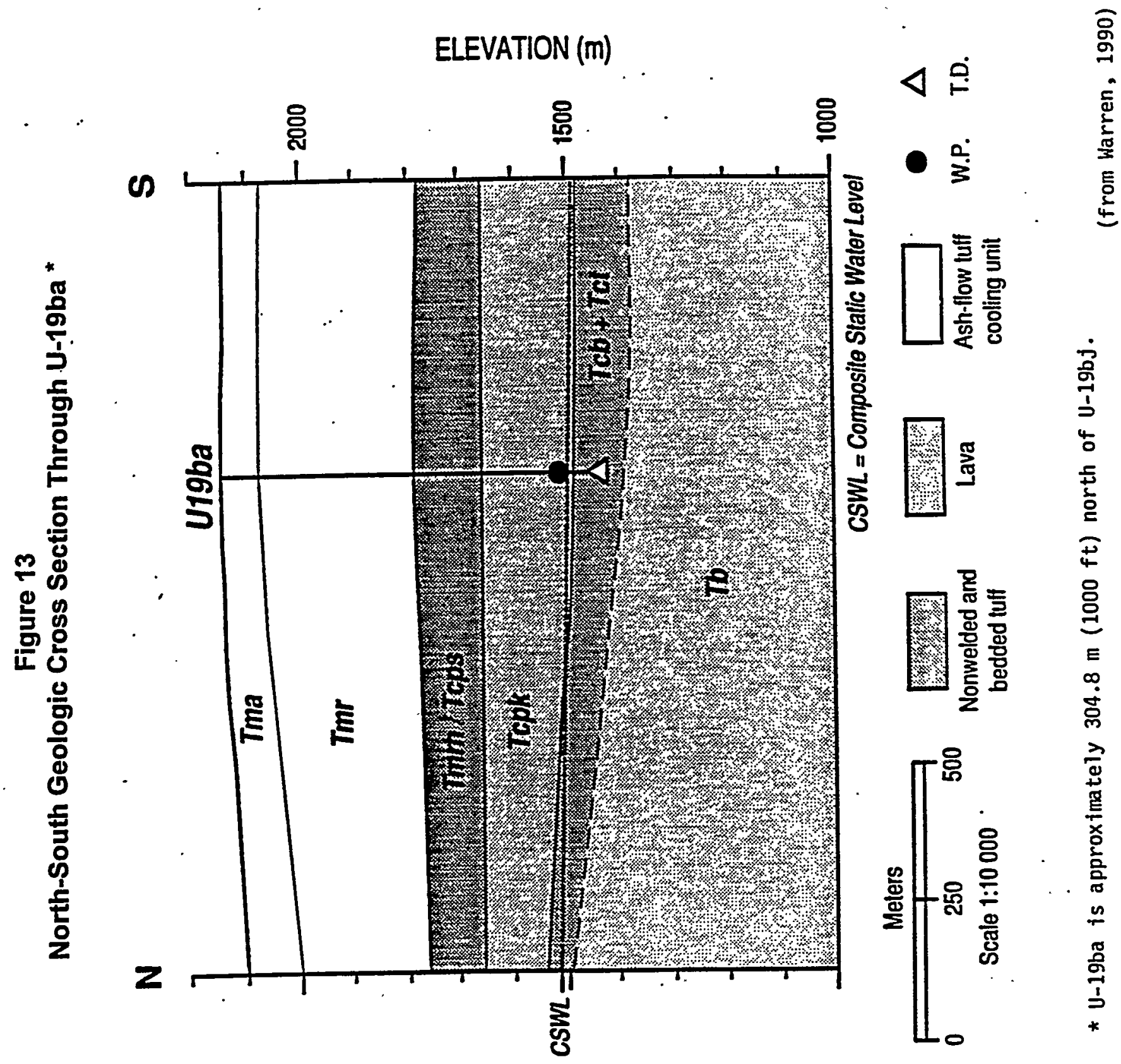
This page intentionally left blank. 


\section{REFERENCES CITED}

Allen, B. M., 1994, Preliminary Geologic Site Characterization for Drill Hole U-1g, Yucca Flat, Nevada Test Site, Raytheon Services Nevada Geologic Report TSP:DGP:112:94, 34 p.

Allen, B. M., 1995, Preliminary Geologic Site Characterization for the Lyner Horizontal Drift Complex, Yucca Flat, Nevada Test Site, Raytheon Services Nevada Geologic Report TSP:DGP:080:95, $71 \mathrm{p}$.

Allen, B. M., 1996, Preliminary Geologic Site Characterization of the Lyner U1A.100 and U1A.101 drifts, Yucca Flat, Nevada Test Site, Bechtel Nevada, 33 p.

Bechtel Nevada, 1997, Bechtel Nevada Support of the 208th Containment Evaluation Panel Meeting, a DOE/NV Stockpile Stewardship Test Readiness Exercise. Bechtel Nevada Project Report, June 25, 1997.

Blankennagel, R. K.; and J. E. Weir, Jr., 1973, Geohydrology of the Eastern Part of Pahute Mesa, Nevada Test Site, Nye County, Nevada, U.S. Geological Survey Professional Paper 712-B.

BN, see Bechtel Nevada.

Cole, J. C. and A. G. Harris, 1996, Stratigraphic and Structural Interpretations of Paleontologic Studies and Core Logging ER-6-1 and ER-6-2 Wells, Nevada Test Site, USGS-GD Assessment Task WB 1.4.1.2.1.02.01.06, 9 p.'

DOE, see U. S. Department of Energy.

Drellack, S. L., Jr., 1990, to R. G. Warren, Los Alamos National Laboratory, Subject: "Geology of Emplacement Hole U-19ba," Fenix \& Scisson of Nevada memorandum number GEO-1060, 19 September 1990.

Drellack, S. L., Jr., 1991, to W. Hawkins, Los Alamos National Laboratory, Subject: "Wireline Sidewall Coring Operations at U-19bg," Raytheon Services Nevada memorandum number GEO-1286, 26 November 1991.

Drellack, S. L., L. B. Prothro, R. L. McCall, and P. H. Thompson, 1992, Preliminary Geology and Drillhole Data Report for Groundwater Characterization Well ER-6-1, Nevada Test Site, Nye County, Nevada, Raytheon Services Nevada, Las Vegas, Nevada.

Ferguson, J. F., A. H. Cogbill and R. G. Warren, 1994, "A Geophysical-Geological Transect of the Silent Canyon Caldera Complex, Pahute Mesa, Nevada." In Journal of Geophysical Research, v. 99, no. 33, p 4323-4339.

IT, see IT Corporation: 


\section{REFERENCED CITED (cont.)}

IT Corporation, 1995, Wells ER-6-1 and ER-6-2 Core Fracture Analyses and Geophysical Log Comparisons, IT Corporation report, Las Vevas, NV, $45 \mathrm{p}$.

McCall, R. L., 1993, Preliminary Geologic Site Characterization for Emplacement Hole U-7cd and U-7cd Sat \#I, Yucca Flat, Nevada Test Site, Raytheon Services Nevada, Las Vegas, NV, $60 \mathrm{p}$.

Noble, D. C., R. D. Krushensky, E. J. McKay, and J. R. Ege, 1967, Geologic Map of the Dead Horse Flat Quadrangle, Nye County, Nevada, U.S. Geological Survey Quadrangle Map GQ614, Scale 1:24,000.

Prothro, L. B., 1991a, to D. Krier, Los Alamos National Laboratory, Subject: "Sandia Camera Run at U-19bg," Raytheon Services Nevada memorandum number GEO-1145, 27 February 1991.

Prothro, L. B., 1991b, to R. G. Warren, Los Alamos National Laboratory, Subject: "BIS Run at U-19bg," Raytheon Services Nevada memorandum number GEO-1172, 30 April 1991.

Prothro, L. B., 1991c, to R. G. Warren, Los Alamos National Laboratory, Subject: "U-19bg LLNL 16 mm Film," Raytheon Services Nevada memorandum number GEO-1184, 15 May 1991.

Prothro, L. B., 1991d, to R. G. Warren, Los Alamos National Laboratory, Subject: "U-19bg Percussion Gun Sampling Operation," Raytheon Services Nevada memorandum number GEO-1213, 8 July 1991.

Prothro, L. B., 1991e, to R. G. Warren, Los Alamos National Laboratory, Subject: "AWS Wireline Sidewall Coring Operation at U-19bg," Raytheon Services Nevada memorandum number GEO-1236, 5 August 1991.

Prothro, L. B., 1991f, to R. G. Warren, Los Alamos National Laboratory, Subject: "Geology of U-19bg Satellite Hole \#1," Raytheon Services Nevada memorandum number GEO-1239, 12 August 1991.

Prothro, L. B., 1991g, to R. G. Warren, Los Alamos National Laboratory, Subject: "Geology of Emplacement Hole U-19bg," Raytheon Services Nevada memorandum GEO-1240, 14 August 1991.

Prothro, L. B., 1991h, to R. G. Warren, Los Alamos National Laboratory, Subject: "U-19bg Samples for Water Content, Bulk Density, and Grain Density Measurements," Raytheon Services Nevada memorandum number GEO-1254, 4 September 1991. 


\section{REFERENCED CITED (cont)}

Prothro, L. B., 1992, to N. Marusak, Los Alamos National Laboratory, Subject: "Sandia Camera Run at U-19bj," Raytheon Services Nevada memorandum number GEO-1427, 9 November 1992.

Prothro, L. B., 1995, Preliminary Geologic Site Characterization for Emplacement Hole U-19bh, Pahute Mesa, Nevada Test Site: Raytheon Services Nevada, 55 p.

Rayburn, C., 1989, to D. Krier, Los Alamos National Laboratory, Subject: "U-19bf Hole Caving," Fenix \& Scisson of Nevada memorandum number GEO-0842, 6 November 1989.

Rayburn, C., 1990, to K. Hahn, Los Alamos National Laboratory, Subject: "Update on U-19bf Geology," Fenix \& Scisson of Nevada memorandum number GEO-0891, 8 January 1990.

Raytheon Services Nevada, 1991, U-19bf Hole History, Las Vegas, NV.

Raytheon Services Nevada, 1993, Department Procedure NTS-GEO-003, Revision 1, "Desription of Rock Samples," effective date, 5 November 1993. Approved as a Bechtel Nevada department procedure with the same number and title on 15 December 1995.

RSN, see Raytheon Services Nevada.

Schmeltzer, J. S., L. E. Barker and D. O. Blout, 1996, Site Characterization Data from the U3ax/bl Exploratory Boreholes at the Nevada Test Site, Bechtel Nevada Report DOE/NV/11718-003.

U. S. Department of Energy, 1995, Completion Report for Well ER-3-2, DOE/NV-408

U. S. Department of Energy, 1997, Completion Report for Wells ER-6-1 and ER-6-1 Satellite Hole \#1. Interim data report available from BN Geology/Hydrology, Mercury, NV.

Warren, R. G., to W. L. Hawkins, Los Alamos National Laboratory, Subject: "Preliminary Geologic Report for U-19ba," Los Alamos National Laboratory memorandum, 5 November 1990.

Warren, R. G., 1991, to distribution, Subject: "Site Characterization Report for U-19bg," Los Alamos National Laboratory memorandum, 8 July 1991. 


\section{Distribution List}

Copies

DOE Office of Scientific and Technical Information, Oak Ridge, TN 2 DOE/NV Technical Information Resource Center, Las Vegas, NV 1 DOE/NV Public Reading Facility, Las Vegas, NV 1

R. M. Bangerter, DOE/ERD, Las Vegas, NV 1

D. W. Duncan, DOE/ESHD, Las Vegas, NV 1

J. E. Magner, USGS Geologic Data Center, Mercury, NV 1

D. A. Trudeau, USGS/WRD, Las Vegas, NV 1

G. A. Pawloski, LLNL, Livermore, CA 1

J. Craig, LANL, Los Alamos, NM 1

W. M. Brunish, LANL, Los Alamos, NM 1

W. L. Hawkins, LANL, Los Alamos, NM 1

R. G. Warren, LANL, Los Alamos, NM 1

Library, IT Corp., Las Vegas, NV 1

R. L. McCall, IT, Las Vegas, NV 1

B. M. Allen, BN, Mercury, NV 1

H. S. Bensinger, BN, Las Vegas, NV 1

B. L. Dozier, BN, Las Vegas, NV 1

S. L. Drellack, BN, Las Vegas, NV 1

H. M. Noto, BN, Mercury, NV 1

J. L. Gonzales, BN, Mercury, NV · 1

H. E. Huckins-Gang, BN, Mercury, NV ' 1

J. P. Manning, BN, Los Alamos, NM 1

J. G. Metcalf, BN, Mercury, NV 1

P. K. Ortego, BN, Las Vegas, NV 1

L. B. Prothro, BN, Mercury, NV 1

M. J. Townsend, BN, Mercury, NV 1 


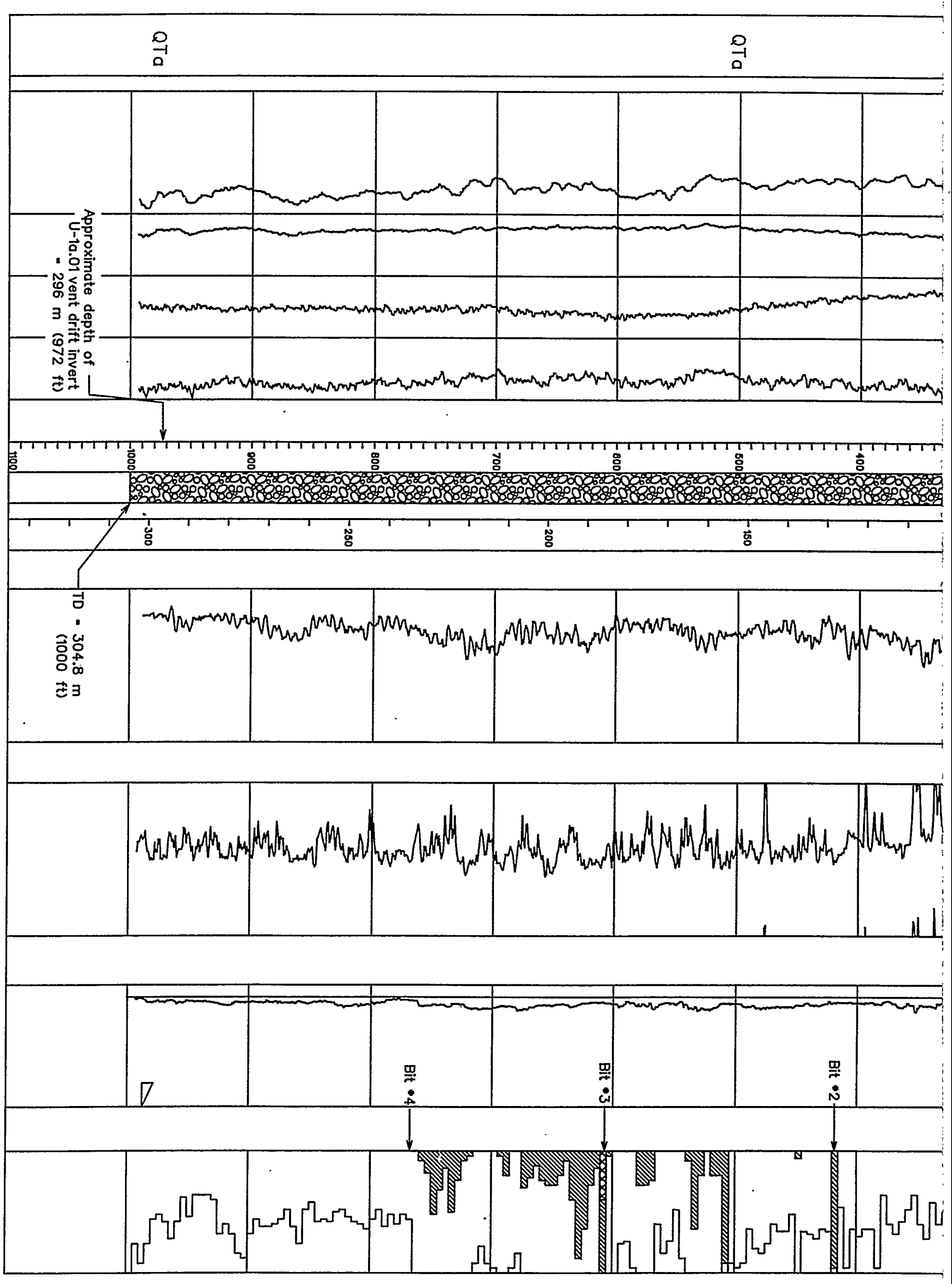

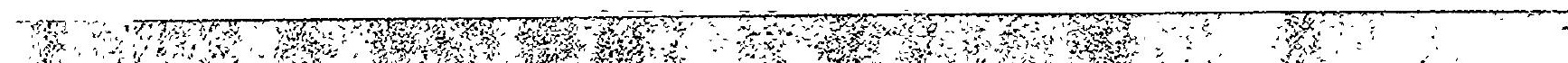




\section{Abridged drill hole statistics for emplacement hole U-7cd.}

Location

Coordinates: N 258,762.6 E 207,680.6 (m)

$N 849,000.24$ E $681.399 .94(\mathrm{ft})$

Elevation: $1254.1(\mathrm{~m}) 4114.7(\mathrm{ft})$

Drilling Data

Spud Date: 2-13-92 (Auger 非); 3-5-92 (Ideco 2500)

Hole Diameter: $3.05 \mathrm{~m}(120 \mathrm{in})$ to $36.0 \mathrm{~m}(118.0 \mathrm{ft})$ : $2.44 \mathrm{~m}(96 \mathrm{in}) 495.3 \mathrm{~m}(1625 \mathrm{ft})$

Total Depth (TO): $495.3 \mathrm{~m}(1625 \mathrm{ft})$

Date Reached TD: $4-14-92$

Drilling Technique: Rotary using air/water and dual reverse circulation

Drilling Problems: None

Casing Record

Surface: From surface to $35.7 \mathrm{~m}(117 \mathrm{ft}), 2.49 \mathrm{~m}$ (98 in) ID

Purpose of Hole: Emplacement

\section{Explanation}

$$
v \quad \text { vitric }
$$

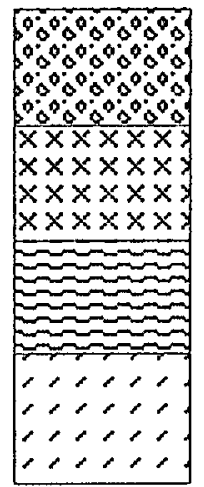

A T luvium

c Calcareous

Ash Flow

Tuff

Bedded

Tuff

Air FaTT

Tuff

s Silicified

vp Vapor-phase mineralization

dv Devitrified

z Zeolitized

m Mafic-rich

Geophysical Logs by Atlas Wireline Services

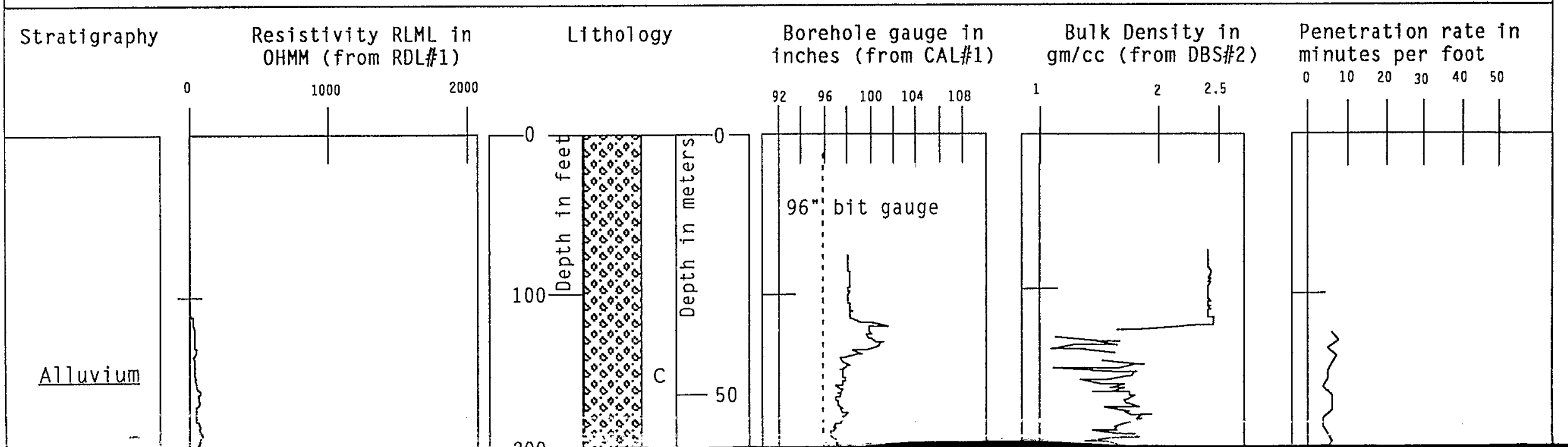




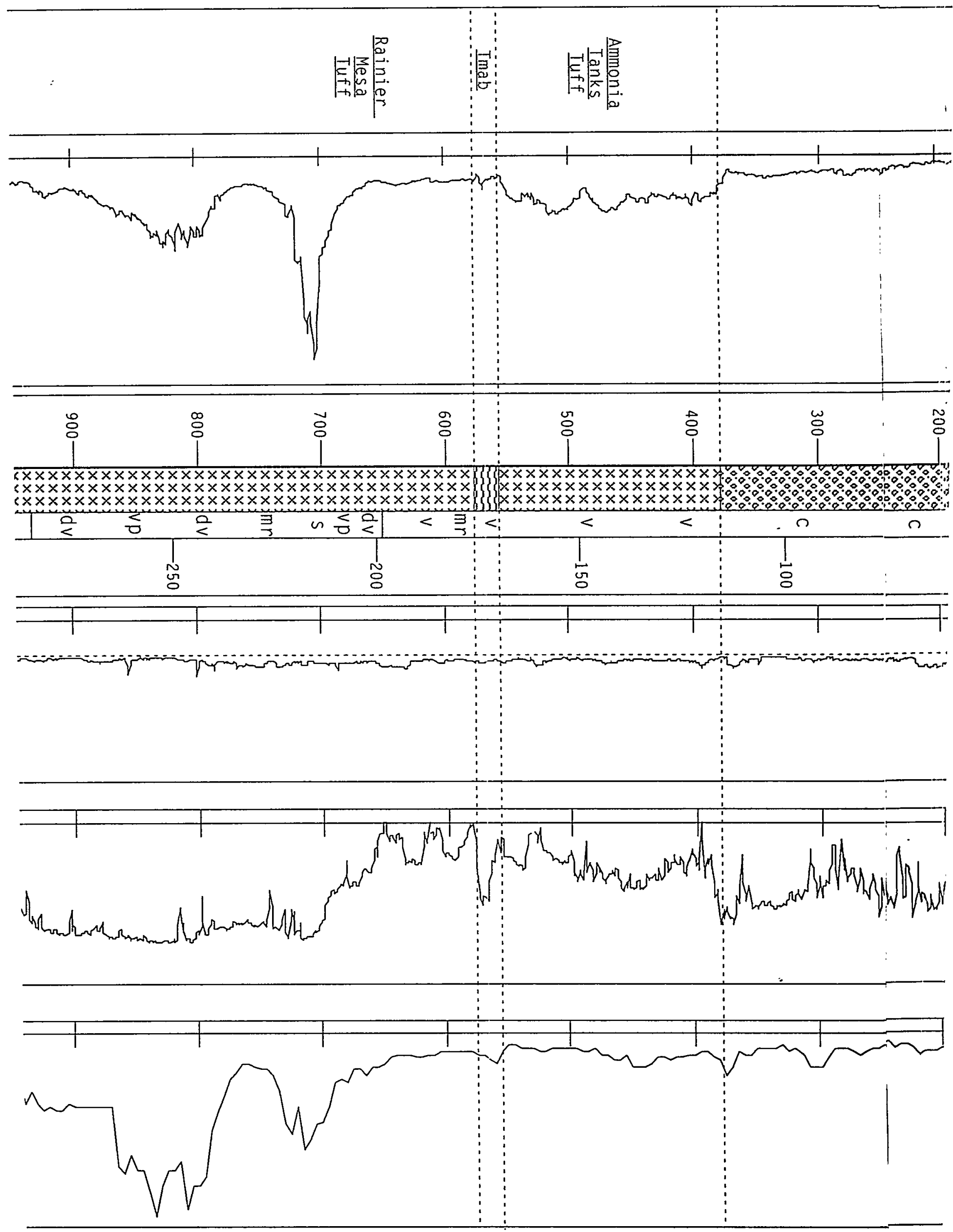




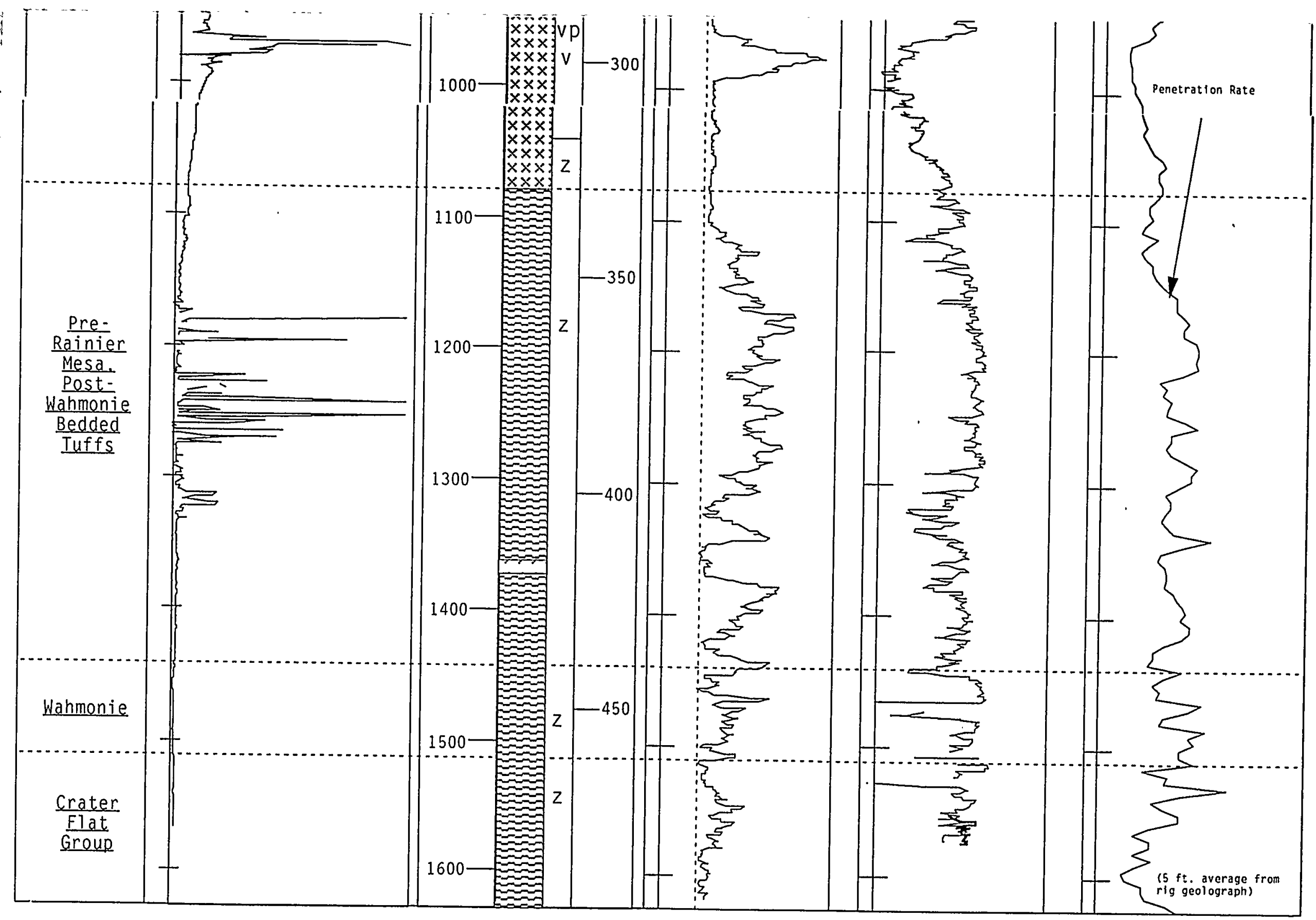




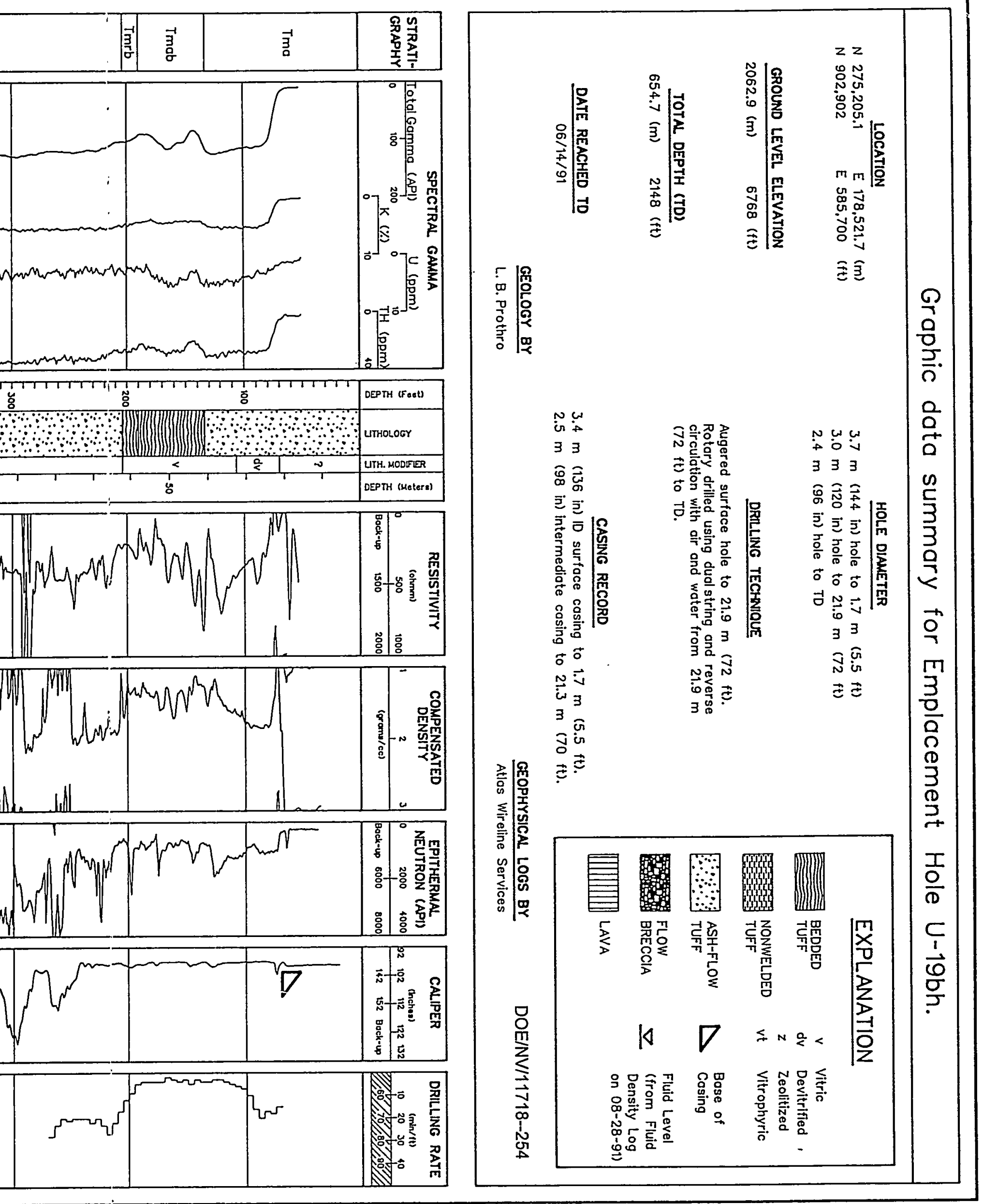


